\title{
Displacement Data Assimilation
}

\author{
Steven Rosenthal ${ }^{1}$, Shankar Venkataramani ${ }^{2}$, Arthur J. Mariano ${ }^{3}$, Juan M. \\ Restrepo $^{4 *}$ \\ 1 Pacific Northwest Laboratory, Richland WA USA 99354 \\ 2 Department of Mathematics and Program in Applied Mathematics, University of Arizona, \\ Tucson AZ USA 85721 \\ ${ }^{3}$ Rosenstiel School of Marine 83 Atmospheric Science, University of Miami, Miami FL \\ USA 33149 \\ ${ }^{4}$ Department of Mathematics, Oregon State University, Corvallis OR USA 97331
}

\begin{abstract}
We show that modifying a Bayesian data assimilation scheme by incorporating kinematically-consistent displacement corrections produces a scheme that is demonstrably better at estimating partially observed state vectors in a setting where feature information is important. While the displacement transformation is generic, here we implement it within an ensemble Kalman Filter framework and demonstrate its effectiveness in tracking stochastically perturbed vortices.
\end{abstract} Keywords: displacement assimilation, data assimilation, uncertainty quantification, ensemble Kalman filter, vortex dynamics

2010 MSC: 93E11, 93B40, 76B47, 37N10

\section{Bayesian Estimation and Displacement Assimilation}

Most sequential estimation strategies are Bayesian. They seek to find estimates of moments of the posterior probability density function of the random, time-dependent, state variable $X(t)$, subject to noisy observations of the state, $Y(t)$. The posterior probability is thus

$$
P(X \mid Y) \propto P(Y \mid X) P(X)
$$

\footnotetext{
* Corresponding Author:

Email address: restrepo@math.oregonstate.edu (Juan M. Restrepo ${ }^{4}$ )
} 
where the first term on the right hand side is the likelihood, and is informed by observations. $P(X)$ is the prior, which is informed by the model. The model is usually an evolution equation for $X$. The model "error" might be an explicit 5 stochastic term representing uncertainties in the evolution equation or its initial or boundary data. The observations are usually stochastic because there are inherent errors in the measurements. When the prior and the likelihood are Gaussian (or a product of Gaussians), minimizing the sum of thee quadratic arguments of $P(Y \mid X)$ and $P(X)$ (equivalently $\log P(Y \mid X)+\log P(X))$ maximizes $P(X \mid Y)$. Moreover, it does so by taking into account the relative certainties in the model and in the measurements. The most familiar sequential estimation technique is the Kalman Filter (see [1]). It seeks a minimizer of the trace of the posterior covariance. It yields an optimal estimate of the time dependent posterior mean and covariance of $X(t)$, over some interval in $t$ when the stochasticity

15 is normally distributed, the dynamics are linear, and relationship between the observations and the state is a linear transformation.

The extended Kalman Filter (see [1]) and the ensemble Kalman Filter (enKF) [2] are frequently-used in mildly non-linear problems with inherently modal statistical distributions. They are not guaranteed to converge without exceptions. ${ }_{20}$ The enKF is very popular since it is relatively easy to implement. It will be employed in this study, as it provides a useful and familiar framework for developing more targeted assimilation methods. The enKF strategy is, like the Kalman Filter approach, a two-stage sequential estimation process. There is a forecast, wherein the model is advanced from $t-\delta t$ to $t$. An ensemble forecast is computed (the model is usually not linearized) by perturbing slightly the state at $t-\delta t$. This is then followed by an analysis stage, which is the Gaussian update from the Kalman scheme. In the absence of measurements, the best estimate is the sample mean and covariance of the ensemble forecast. (This presumes very critically that the mean of forecast errors remains close to zero). 30 If observations are available at that time $t$, these are individually assimilated into each ensemble member. Rather than solving the update of the covariance matrix, one uses a sample covariance based on the ensemble of forecasts. More 
details on the enKF filtering scheme appear in Appendix B

Most data assimilation schemes are variance-minimizing; they give an appropriately weighted average of the predictions of the model and the noisy observations [3. These methods thus tend to decrease sharp gradients, consequently smearing or obliterating "features" (vortices, shock-fronts, etc.) in the state variable being estimated. If tracking the features are critical, a purely varianceminimizing methodology will produce estimates that might not be accurate enough for prediction, particularly in problems where capturing characteristics is critical, as in wave propagation problems. In this paper we propose a modification to sequential state estimation that can improve estimates where features are important. We denote this two-state data assimilation strategy displacement assimilation. Many of the sequential data assimilation procedures yield 45 an estimate of $X(t)$ that may not be in the solution space of the model; for that matter, it might not even be physical. Constraints may be added to the Bayesian statement to promote physically reasonable properties in the analysis estimate, which motivates inserting an extra step into the data assimilation process. The method developed here applies this strategy to improve the estimates of a variance minimizing strategy when morphological features in the state variable are important. For example, suppose we want to estimate characteristic paths for a wave process. These paths are both space and time dependent (phase dependent). If designed properly, an assimilation method that makes a phase correction in addition to a blending of observations and model outcomes might deliver better estimates of such things, as the space-time information of what generated a wave, or the space-time information that better tracks the wave characteristics. This two-stage process is what we call displacement assimilation.

If the displacement correction and the estimation process are kept separate, 60 it is possible to develop a displacement correction scheme that could then be applied to a variety of different estimation strategies. The development we present here is in that spirit. In this study we will be contrasting the standard enKF and something we call displacement enKF, wherein the difference is that 
in the latter we add a phase correction.

Adding phase corrections within the context of data assimilation is not a new idea. Among other works, we can mention [4, who argued how this procedure might improve predictions in meteorology (see also [5], [6]). Ravella and collaborators [7] applied variational techniques (3DVAR) to jointly optimize displacement and amplitude corrections, and improve alignment of vortex-like features. Recently, displacement assimilation has been incorporated into the spatio-temporal data assimilation capabilities (4DVAR) of the weather forecasting tool WRF DAS for short-term hurricane prediction. 8]

Percival used ideas from control theory to find coordinate transformations that could improve predictions. 9. He applied area preserving flows whose limit 75 in a pseudo-time variable defines a phase correcting map that locally minimizes a Bayesian-type penalty functional. In contrast, we will use area preserving maps to do the position correction "in one shot" every time new data is assimilated into the model.

An additional criterion in our methodology is to ensure that the phase corrections are kinematically consistent with the underlying physics of the system. This is not always necessary for making phase corrections. Frazin [10, for example, makes phase corrections that have no physical basis - the aim of his work is to improve the optical data using a data assimilation system. In our context however, it is critical that the corrections be consistent with the under-

${ }_{85}$ lying physics, because we are using the phase corrections to capture the effects of unresolved/unmodeled (but physically constrained) effects in the system.

The key in regularizing (or imposing) convexity in the space of displacement maps is to invoke physics or some ad-hoc constraint. We suggest the use of a physically-motivated constraint. When compared to an ad-hoc constraint, a 90 physics-motivated constraint leads to estimates whose optimality can be understood on physical grounds. Intuitive approaches to displacement corrections can be found in early works by Brewster [5, 6]. This strategy is formalized mathematically by Ravela 17, 11, who proposes that the optimal map be constructed so as to minimize the wave number of the displacement. The present work pro- 
poses an alternative form and implementation of these constraints, pursuing the same motivation.

\section{Displacement Assimilation, Defined}

Consider the problem of determining the state $y$ which best explains a set of noisy observations $d=h\left(y^{t}\right)+\epsilon$ of an unknown true state $y^{t}$, where the nonlinear observation operator can be modeled by $h(y)$ and the observation noise $\epsilon \sim \mathcal{N}(0, R)$. We want to solve the ill-posed problem $d=h(y)$, and resort to minimizing the likelihood functional,

$$
\mathcal{J}_{L}[y]=\|d-h(y)\|_{R^{-1}}^{2}
$$

where the so-called maximum likelihood estimator is the maximizer of $\exp ^{-\mathcal{J}_{L}[y]}$. Next, presume we have prior information that $y^{t} \sim \mathcal{N}\left(y^{f}, P^{f}\right)$, where $y^{f}$ is a forecast mean, and $P^{f}$ the forecast covariance. The maximum-a-posteriori estimator is the maximizer of $\exp ^{-\mathcal{J}[y]}$, where

$$
\mathcal{J}[y]=\mathcal{J}_{L}[y]+\left\|y-y^{f}\right\|_{\left(P^{f}\right)^{-1}}^{2}
$$

We are penalizing the distance from the forecast $y^{f}$ in the matrix-weighted norm defined by the inverse of the forecast covariance, $\left(P^{f}\right)^{-1}$. The covariance matrices in real applications are often singular, by design or to machine precision. This is equivalent to the so-called Bayesian formulation of the inverse problem; conditioning on a prior probability distribution is equivalent to the addition of a regularizing term.

While the distribution of a dependent variable such as vorticity depends on the underlying distribution of spatial displacements of the vorticies, typically displacement assimilation is rendered explict by correcting for displacement, and then correcting the dependent variables. In general, our algorithm applies a regularized (Bayesian) optimization procedure to both steps. If $y_{a}$ is a displacement-corrected model field, and $a$ the parameters identifying the 
tially/iteratively the two penalty functionals,

$$
\begin{aligned}
& \mathcal{J}_{p}[a]=\left\|d-h_{a}(a)\right\|_{R^{-1}}^{2}+\left\|a-a^{f}\right\|_{\left(P_{a}^{f}\right)^{-1}}^{2} \\
& \mathcal{J}_{a}[y]=\left\|d-h_{y}\left(y_{a}\right)\right\|_{R^{-1}}^{2}+\left\|y_{a}-y_{a}^{f}\right\|_{\left(P_{y_{a}}^{f}\right)^{-1}}^{2}
\end{aligned}
$$

We are taking advantage of the fact that we can use a given set of observations $d$ and likelihood covariance $R$ more than once: both for the displacement parameters and the corrected model fields. Remaining undefined are the ob-

\section{Displacement Assimilation in Ensemble Vortex Tracking}

The net effect of large-scale forcing on a system of interacting coherent vortices is two-fold. The dominant effect is in modifying the trajectories of the vortex centers. A much smaller effect is changing the shape/structure of individual vortices [12]. This naturally suggests the use of statistically derived filtering algorithms to track the trajectories of the vortices for data assimilation. The trajectories of interacting vortices are highly sensitive to changes in their relative displacement and amplitude, and thus present a useful "toy model" for developing and testing our displacement assimilation methodology. We will use a stochastic barotropic vorticity equation to compare the enKF estimates with and without displacement adjustments. Our model is noisy; we do not use the ideal vorticity equation. The added noise accounts for unresolved or ignored physical processes in the actual physical system. Furthermore, there can also be uncertainties in initial and boundary conditions. The stochastic vorticity model solutions are therefore realizations of the stochastic process, drawn from 135 a probability distribution. 
Consider an ideal incompressible fluid in a two-dimensional domain $D \subset \mathbb{R}^{2}$. Denote the velocity vector field $V=(u, v)$. Assume this velocity is incompressible, $\nabla \cdot V=0$. The vorticity associated with the velocity field is $\omega=\nabla \times V$. Let the stream function be $\psi=\left(-\frac{\partial \psi}{\partial y}, \frac{\partial \psi}{\partial x}\right)$. The stochastic model for the vorticity 140 is

$$
\begin{aligned}
\partial_{t} \omega+V \cdot \nabla \omega & =\delta f, \quad \omega: D \times[0, \infty) \rightarrow \mathbb{R}, \\
\omega(z, 0) & =\omega_{0}(z)+\delta \omega_{0}(z), \\
\nabla^{2} \psi & =\omega, \quad \psi: D \rightarrow \mathbb{R} \\
\nabla \psi \cdot \partial D & =\delta b
\end{aligned}
$$

where $\delta f, \delta \omega_{0}$, and $\delta b$ are perturbations in the forcing, initial condition, and boundary condition with known probability distributions. Perturbations in initial condition and boundary conditions will not be invoked in this study. The value of the stream function, along the boundary of the domain, $\partial D$ is prescribed. Note that (6) couples the the vorticity and stream functions, and consequently couples a set of vorticity features to their Lagrangian paths. The Poisson equation (6) is linear, so it maps perturbations in vorticity features directly into perturbations in their trajectory. Accumulated noise in nearby features mutually affects the trajectories of the vortices. Conversely, in systems constrained by diagnostic relationships such as (6), studying spatial displacements in features provides a way to correct the accumulated errors in their amplitude, which improves the subsequent prediction of their future path. Hence, the importance of the forcing error term $\delta f$ which can generate random local changes in the intensity of features, as well as nudge the positions of nearby features.

Appendix C gives details on the parameter values for the calculations that follow. They were chosen to provide a system of spatially continuous features which experience significant position errors while retaining enough structure to persist throughout the simulation. The initial condition defines a system of co-rotating vortices. In Figure 1 two known $\left(\delta \omega_{0}=0\right)$ and initially identical 
vorticity pulses are mutually advected in a bounded rectangular domain. The boundary conditions enforce a zero-outflow condition $(\delta b=0)$ that serves two purposes. The first is to ensure features cannot leave the domain, so that the complexity of the tracking problem remains consistent over time and between

\subsection{Model Setup and Observations}

In the stochastic vorticity dynamics we assume a (deterministic) initial vorticity condition $\omega(z, 0)=\omega_{0}(z)$ for $z \in D=\left[x_{L}, x_{U}\right] \times\left[y_{L}, y_{U}\right]$. The vorticity trajectory $\omega: D \times[0, \infty) \rightarrow \mathbb{R}$ is given by

$$
\frac{\partial \omega}{\partial t}=-V \cdot \nabla \omega+B_{\omega}(z) \cdot \xi
$$



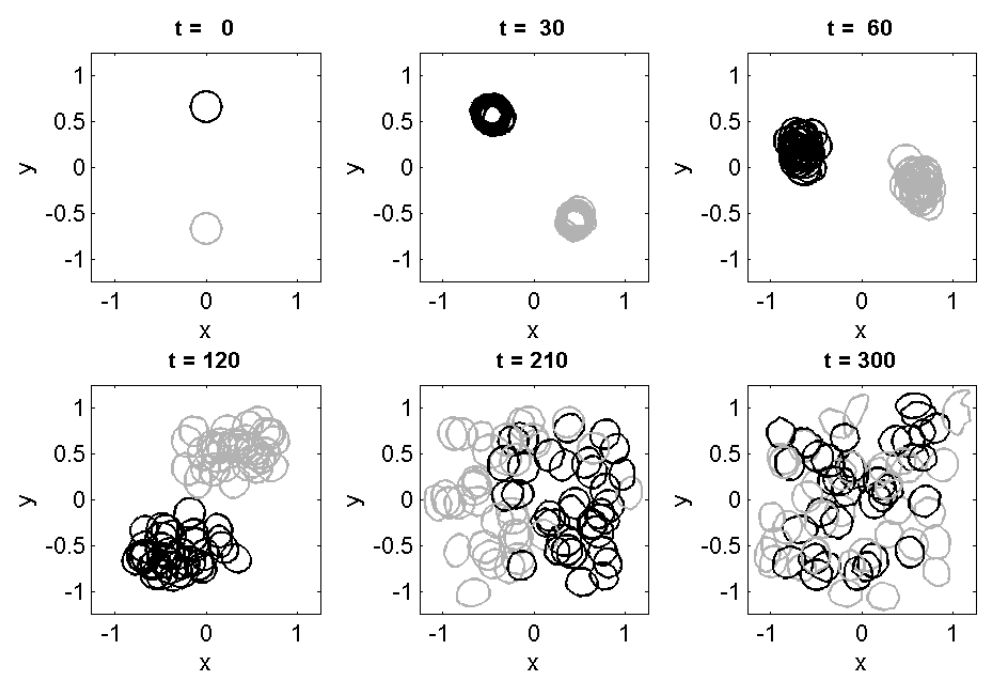

Figure 1: The noise regime of the model. A sample of 35 realizations of the vorticity model, with equal-height $(\omega=.5)$ contours displayed.

Equation (7) is subject to zero-outflow boundary condition $\nabla \Psi \cdot \partial D=0$, where $\Psi: D \rightarrow \mathbb{R}$ is the stream function corresponding to $\omega$. The model error forcing term $\delta f$ is defined by a Gaussian random function with spatial covariance factor $B_{\omega}(z)$, and white noise process $\xi \sim \mathcal{N}\left(0, I_{r}\right)$. Equation 77 is approximated on a uniform rectangular grid on $D$ with $N_{x}$ and $N_{y}$ subintervals in the $\{x, u\}$ and $\{y, v\}$ dimensions, respectively, and the vorticity, velocity, and stream function grids are aligned. Then the total dimensionality of the problem is $N=\left(N_{x}+1\right)\left(N_{y}+1\right)$. This stochastic differential equation (SDE) is integrated in time using the stochastic Heun method [13. Each time the right-hand side of Equation (7) is evaluated, the stream function is obtained by solving the Poisson equation $\Delta \Psi=\omega$ via a second-order central difference discretization of the Laplacian. The velocity components are computed using second-order centered difference approximations of the derivatives.

The structure of $B_{\omega}$ and dimension $r$ of the Gaussian random function driving the model noise are inferred from assumptions on the variability of the velocity components. Since vorticity is a derived state parameter, we make an 
effort to define a (reasonable) model uncertainty based only on the (given) covariance statistics of the observable state variables, i.e. the velocity vector field components. The noise in the two velocity components is assumed mutually independent and spatially distributed with a squared-exponential correlation structure,

$$
k_{u}\left(z_{1}, z_{2}\right)=k_{v}\left(z_{1}, z_{2}\right)=\sigma_{V}^{2} \exp \left(-r_{V}^{-2}\left\|z_{2}-z_{1}\right\|_{2}^{2}\right)
$$

where $\sigma_{V}^{2}$ and $r_{V}$ are the scale and shape parameters, respectively, setting the pointwise variance and decorrelation length of the model error kernels $k_{u}$ and $k_{v}$. Let $Q_{V}$ denote the Gramian matrix corresponding to the evaluation of the kernels on the discretized domain $D$, and compute its factorization $Q_{V}=B_{V} B_{V}^{T}$, where $\operatorname{rank}\left(B_{V}\right)$ is sufficient to represent all numerically significant modes of the original covariance matrix. Each spatial mode of each velocity component is then dampened to zero at the boundaries in the outflow directions, so that the model perturbations are guaranteed consistent with the boundary conditions. Specifically, another shape parameter $r_{b}$ is introduced to scale the width of the transition region. Then the following bump function is defined,

$$
\left[1-\exp \left(-\frac{\left|x-x_{L}\right|}{r_{b}}\right)\right] \cdot\left[1-\exp \left(-\frac{\left|x-x_{U}\right|}{r_{b}}\right)\right]
$$

which is unity in $\left[x_{L}+r_{b}, x_{U}-r_{b}\right]$, and scales the $u$ model noise modes. A similar bump function in the $y$ dimension is defined and applied to the $v$ modes. With consistent velocity perturbations, the vorticity perturbation modes can be computed from the definition $\omega=\partial_{x} v-\partial_{y} u$,

$$
\delta \omega=B_{\omega} \cdot \xi=\left[\begin{array}{ll}
\frac{\partial B_{v}}{\partial x} & -\frac{\partial B_{u}}{\partial y}
\end{array}\right]^{T} \cdot\left(\begin{array}{ll}
\xi_{u} & \xi_{v}
\end{array}\right)
$$

where the derivatives are approximated by centered differences. The vorticity model perturbations driving the SDE in Equation (7) follow a Gaussian random function with spatial covariance $Q_{\omega}=B_{\omega} B_{\omega}^{T}$. We should keep in mind that this covariance actually measures noise from velocity perturbations in a $r=2 \cdot \operatorname{rank}\left(B_{V}\right)$-dimensional (observation) space expressed in $r / 2$-dimensional vorticity model space. 
In our simulations, the initial condition was deterministic and consisted of two co-rotating vortices,

$$
\omega_{0}(z)=a_{1} \cos ^{2}\left[\frac{\pi}{2} r_{1}(z)\right]+a_{2} \cos ^{2}\left[\frac{\pi}{2} r_{2}(z)\right]
$$

where the rescaled radius $r_{j}(z)$ defines the support of each vortex, and is given by

$$
r_{j}(z)=\frac{d_{j}(z)}{r_{s, j}} \chi_{\left\{d_{j}(z) \leq r_{s, j}\right\}}(z)
$$

and $d_{j}(z)=\left[\left(x-x_{c, j}\right)^{2}+\left(y-y_{c, j}\right)^{2}\right]^{1 / 2}$, the squared distance from the vortex, center, and $\chi_{A}(z)$ is the indicator function for all $z \in A$. The trajectory of one realization of the generalized model, including the initial condition, is provided in Figure 4, with one frame for each assimilation time.

The observations of the state variables in the present example will be a sparse array of noisy Eulerian measurements of the velocity components. The observation operator is a map from the state variables to the measured quantities. In particular, let $h_{a}\left(\omega,\left\{x_{d, j}, y_{d, j}\right\}\right)$ estimate the velocity components at each $\left(x_{d, j}, y_{d, j}\right)$ from the continuous model vorticity field $\omega$. As is standard practice in twin simulation experiments, synthetic observations are generated given a true vorticity field $\omega^{t}$. Then, the velocity components measured are given by $d_{V}:=\left(d_{u}, d_{v}\right)^{T}=h_{a}\left(\omega^{t}\right)+\epsilon_{d}$, where $\epsilon_{d} \sim \mathcal{N}(0, R)$ are the assumed independent and identically distributed normal observation errors, and $R=\tau I_{2 N_{d}}$ is the observation covariance matrix. Note that the locations where velocity is measured will be a rectangular array and a subset of the discretization of the spatial domain $D$, though in principle the observed locations can be any scattered points in $D$.

\subsection{Displacement Correction Via Area-Preserving Maps}

The stochastic vorticity equation produces a distribution of vorticity functions whose features have perturbed amplitudes; this introduces noise in the Lagrangian path of these features. The generalized inverse problem for position errors is to identify which of the possible Lagrangian perturbations would produce a realization of the stochastic vorticity function that most closely matches 
the true state of the system. Consider a quadratic penalty functional that measures the Eulerian distance between the true vorticity function $\omega^{t}$ and various candidate analyses $\omega$ with respect to a specific norm $\|\cdot\|$,

$$
\mathcal{J}_{a}[\omega]=\frac{1}{2}\left\|\omega^{t}(Z)-\omega(Z)\right\|^{2}
$$

In order to relate the forecast prediction to candidate analyses through corrections in the position of features, we define a family of smooth, invertible maps $Z=\Phi(z ; a)$. Throughout the following discussion we will consistently refer to the coordinates native to the true and observed vorticity function by $Z$, and the coordinates on which the forecast model $\omega^{f}$ is defined by $z$. Then we can write $\omega(Z)=\omega^{f}(z)=\omega^{f} \circ \Phi^{-1}(Z ; a)$. Now we can recast 13 in terms of the map parameters,

$$
\mathcal{J}_{p}[a]=\frac{1}{2}\left\|\omega^{t}(Z)-\omega^{f} \circ \Phi^{-1}(Z ; a)\right\|^{2},
$$

which allows us to ask which position corrections provide the best agreement

${ }_{225}$ (minimum $\mathcal{J}_{p}$ ) with measurements of the true vorticity function. If the maps $\Phi$ are to model stream function perturbations, they also must be divergencefree, in the sense that they preserve the total vorticity on any subset as it is mapped from one position to another. These subsets include any closed contour of the vorticity function, including the concentric and parallel contours that describe the geometry of features. Hence, preserving the total vorticity and area on contours is akin to preserving the geometry of features, i.e quantities like volume and intensity.

\subsubsection{Symmetric Parameterization of Displacement Maps}

A standard method from classical mechanics to define area preserving maps 235 is by canonical transformation of the spatial coordinates. However, the transformed coordinates must be implicitly solved. Furthermore, the maps are difficult to parameterize symmetrically, in the sense that spatial symmetries in the map parameters are not necessarily reflected in the transformed coordinates. Instead, under a small-displacement assumption, we can show an approximate 

time. Details and references are provided in Appendix A.

We define area preserving maps $(X, Y)=\Phi(x, y ; a)$ by integrating stream functions over a fixed time interval,

$$
\begin{aligned}
& \Phi(x, y ; a)=(x, y)+ \\
& \int_{0}^{1}\left(-\Psi_{y}[x(t), y(t) ; a], \Psi_{x}[x(t), y(t) ; a]\right) d t
\end{aligned}
$$

The parameterization of the stream function $\Psi$ is linear in $a$, i.e. taking tained simply by making the substitutions $(x, y, a) \rightarrow(X, Y,-a)$. Unlike the usual formulations of canonical transformations, the approach of integrating a flow gives an explicit, rather than an implicit function, and is un-constrained, unlike the constraints on the mixed partial derivatives of a generating function position correction problem, a sequence of maps can be defined through iterative optimization of functionals like Equation (14). In this context, the small displacement assumption is reasonable, and we can proceed to take advantage of the theoretical simplicity of the canonical map structure to develop additional tools. Moreover, to generate the maps we take advantage of the practical convenience of integrating divergence-free flows. Hereafter we identify the map function and stream function, $\Psi(x, y ; a) \leftrightarrow \zeta(X, y ; a)$, and the particular usage will be clear from context. In fact, these two approaches are identical in applications which require the map to be linearized, such as when we project the map parameters. In the next section we apply both points of view in order to derive regularization terms that help provide smooth displacements where feature information is present, based on an application of continuum mechanics.

\subsubsection{Transformation Regularization}

265

In the set of possible feature displacement maps, we want to avoid coordinate transformations which excessively deform the geometry of strong features in the 
model function. In fact, excessive deformations in general should be avoided. Since the likelihood functional will penalize only coordinate transformations where there is significant feature information, then an otherwise unfettered optimization routine can significantly distort regions where there are no features in either the observed or modeled functions. Perturbations in lightly-constrained regions can generate enough of a change in the penalty functional to create local decreases in the objective function that have nothing to do with the displacement of significant features-precisely the non-convexity that regularization schemes, such as Tikhonov regularization, are designed to eliminate.

Suppose the displacement map complexity is kept well below that of the original problem,

$$
\operatorname{dim}(a) \ll \operatorname{dim}(\omega)=\operatorname{dim}(\zeta)=\operatorname{dim}(\Phi)
$$

Denote the linear map from the displacement parameters to vorticity values as $T_{\omega a}$, and denote its pseudoinverse $T_{a \omega}$, the map projecting a vorticity function into displacement parameter space. These maps can be used to estimate a prior model covariance of map parameters from a given prior vorticity covariance. For vorticity perturbations $\delta \omega$ and map parameter perturbations $\delta a$, we can write

$$
\begin{aligned}
P_{\omega} & =\operatorname{Cov}(\delta \omega)=\mathbb{E}\left(\delta \omega \delta \omega^{T}\right) \\
& =\mathbb{E}\left(T_{\omega a} \delta a \delta a^{T} T_{\omega a}^{T}\right) \\
& =T_{\omega a} \mathbb{E}\left(\delta a \delta a^{T}\right) T_{\omega a}^{T} \\
& =T_{\omega a} \operatorname{Cov}(\delta a) T_{\omega a}^{T}=T_{\omega a} P_{a} T_{\omega a}^{T}
\end{aligned}
$$

and

$$
P_{a}=T_{a \omega} T_{\omega a} P_{a} T_{\omega a}^{T} T_{a \omega}^{T}=T_{a \omega} P_{\omega} T_{a \omega}^{T}
$$

Then we can consider regularization terms defined directly in the space of displacement parameters. In particular, we write

$$
J_{p}[a]=\frac{1}{2}\left\|d_{\omega}-\omega^{f} \circ \Phi^{-1}\left(Z_{d}, a\right)\right\|_{R^{-1}}^{2}+S[a ; \alpha]
$$

where we could take the regularization term $S[a]=\left\|a-a^{f}\right\|_{C_{a}^{-1}}^{2}$, the usual quadratic form for the model prior, with the forecast $a^{f}=0$, and the co- 
variance penalty factor $C_{a}$. Note that in practice the functional will not be expressed in terms of direct vorticity observations, $d_{\omega}$, but rather with velocity measurements, $d_{V}$.

There is one impediment to using this regularization - it requires knowledge of $P_{\omega}$, the covariance of the vorticity (or velocity) fluctuations. In a situation where we have knowledge of the vorticity covariance $P_{\omega}$, it is optimal to minimize the functional in eq. 19 . However, $P_{\omega}$ is often not known in practice, because vorticity fluctuations are driven by unresolved/unmodeled physical processes, e.g. sub-grid processes. As an alternative, especially in a situation where we do not have information on the covariance of the vorticity fluctuations, we can choose an ad hoc regularization term which includes more information than just the weighted distance from the forecast, such as a preference for smoothly varying transformation maps. Below, we present one such procedure - strain regularization. We use this ad hoc regularization method, instead of the expression in eq. (19), to demonstrate the applicability of the method, even in situations where we do not have access to the vorticity covariance $P_{\omega}$.

\subsubsection{Strain regularization}

Since we can write down the formula for a coordinate transformation $z=$ $\Phi^{-1}(Z)$, then we can write down the local displacement induced by the transformation, $U(Z)=Z-z(Z)=Z-\Phi^{-1}(Z)$. This is the amount the original coordinate system was deformed to arrive at the current coordinates $Z$. We also can write down how differential length elements in the former system $d z$ are deformed in the new system $d Z$. For sufficiently smooth displacements, and $|\zeta| \ll 1$

$$
\epsilon:=\frac{1}{2}\left[\nabla_{Z} U+\left(\nabla_{Z} U\right)^{T}\right]
$$



simplify to

$$
\begin{aligned}
\epsilon[\zeta] & =\left[\begin{array}{cc}
\epsilon_{X X}[\zeta] & \epsilon_{X Y}[\zeta] \\
\epsilon_{Y X}[\zeta] & \epsilon_{Y Y}[\zeta]
\end{array}\right] \\
& \approx\left[\begin{array}{cc}
-\zeta_{y X} & \frac{1}{2}\left(\zeta_{X X}-\zeta_{y y}\right) \\
\frac{1}{2}\left(\zeta_{X X}-\zeta_{y y}\right) & \zeta_{y X}
\end{array}\right] .
\end{aligned}
$$

For the strain regularization treatment, we augmented (14) with a weighted Frobenius norm of the engineering strain tensor in 21) at each model point,

$$
\begin{aligned}
\|\epsilon[\zeta]\|_{F, \alpha}^{2} & =\frac{\alpha_{n}}{2}\left(\epsilon_{X X}^{2}[\zeta]+\epsilon_{Y Y}^{2}[\zeta]\right) \\
& +\frac{\alpha_{s}}{2}\left(\epsilon_{X Y}^{2}[\zeta]+\alpha_{Y X}^{2}[\zeta]\right) \\
& =\alpha_{n}\left\|\zeta_{y X}\right\|^{2}+\frac{\alpha_{s}}{4}\left\|\zeta_{X X}-\zeta_{y y}\right\|^{2} \\
& \sim \alpha_{n}\left\|\Psi_{y x}\right\|^{2}+\frac{\alpha_{s}}{4}\left\|\Psi_{x x}-\Psi_{y y}\right\|^{2}
\end{aligned}
$$

where $\alpha_{n}$ and $\alpha_{s}$ calibrate the size of the normal and shear strain penalties, respectively. Since there is no prior model distribution limiting the displacement, the optimization algorithm is free to find the true bulk position shift. However, without a calibrated regularization term, the algorithm also is free to suggest local deformations wherever there is not significant feature information. This situation can result from not having any background error at all, as is the case here, or whenever observation uncertainty or scarsity effectively places observation uncertainty on the same or greater scale as the background signal noise.

Provided a linear parameterization of the stream function $\Psi(z)=\sum_{j}^{N} a_{j} B_{j}(z)=$ $B(z) \cdot a$, the strain regularization term can be written as a matrix-weighted norm,

$$
\begin{aligned}
\|\epsilon[\Psi]\|_{F, \alpha}^{2} & =\alpha_{n}\left(B_{y x} \cdot a\right)^{T}\left(B_{y x} \cdot a\right) \\
& +\frac{\alpha_{s}}{4}\left(B_{x x} \cdot a-B_{y y} \cdot a\right)^{T}\left(B_{x x} \cdot a-B_{y y} \cdot a\right) \\
& =\|a\|_{C_{\epsilon, \alpha}^{-1}}^{2}
\end{aligned}
$$

where

$$
C_{\epsilon, \alpha}=\left[\alpha_{n} B_{y x}^{T} \cdot B_{y x}+\frac{\alpha_{s}}{4}\left(B_{x x}-B_{y y}\right)^{T}\left(B_{x x}-B_{y y}\right)\right]^{-1}
$$


If this constraint is simply added to the penalty functional in 190 , the standard least-squares minimizer requires the sum $C_{a}^{-1}+C_{\epsilon, \alpha}^{-1}$, forcing us to explicitly calculate the model prior covariance inverse. Alternatively, we directly regularize the model covariance

$$
C_{a, \alpha}=C_{a}+C_{\epsilon, \alpha}
$$

315 is no problem provided the complexity condition in $(16)$ is maintained, which depends on the manner in which the displacement maps are discretized.

To confirm the efficacy of strain regularization for penalizing excessive deformations while permiting bulk feature position realignment, a numerical opization term (See Figures 2 3). A simple feature modeled after two coalescing vortices was generated with no background error, and a truth feature was generated by a combination of a rigid transformation and amplitude perturbation. The $L^{2}$-norm penalty functional from 13 was used to quantify the residual

\subsubsection{Displacement Algorithm Implementation Details}

No basis has yet been prescribed for representing the displacement function $\zeta$. Typical decorrelation lengths for the amplitude perturbations that generate position errors are not global in scale. It is reasonable to assume noise length 330 scales are no greater than the dominant features in the model. For this reason, historically, local parameterizations of displacement functions have been proposed. Mariano [15] decomposed individual contours into angular sections and analyzed displacement along these directions, while Brewster [6] considered a hierarchical model of discretizations, where first three and then one grid step corrections would be sought. Ravela [7] proposed a bicubic spline representation. Frazin [10], like Brewster, combined approximation with regularization by considering a hierarchical spline model similar to multigrid techniques, where the displacement function estimate is refined on a sequence of increasingly fine scales. 

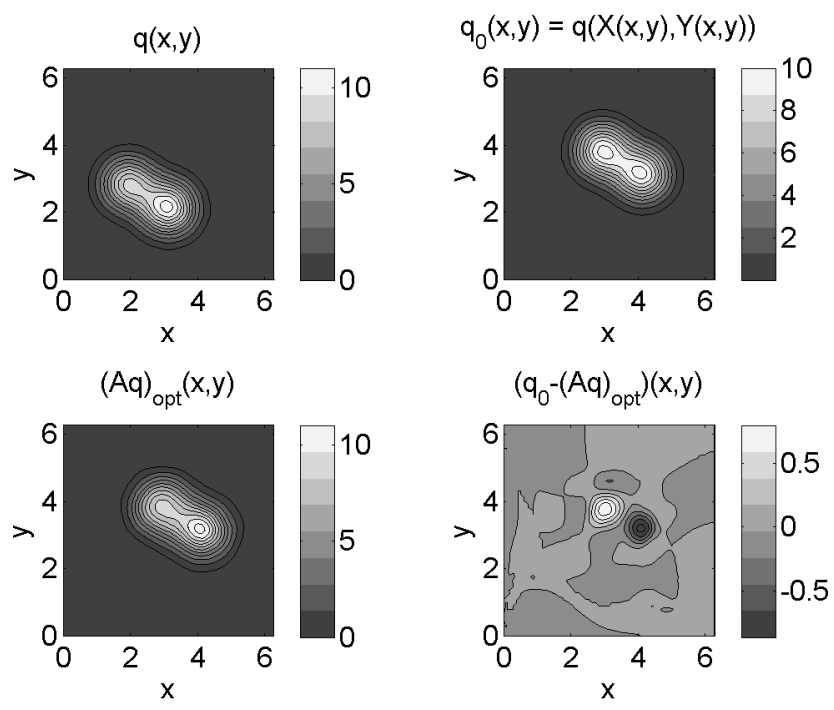

(a)

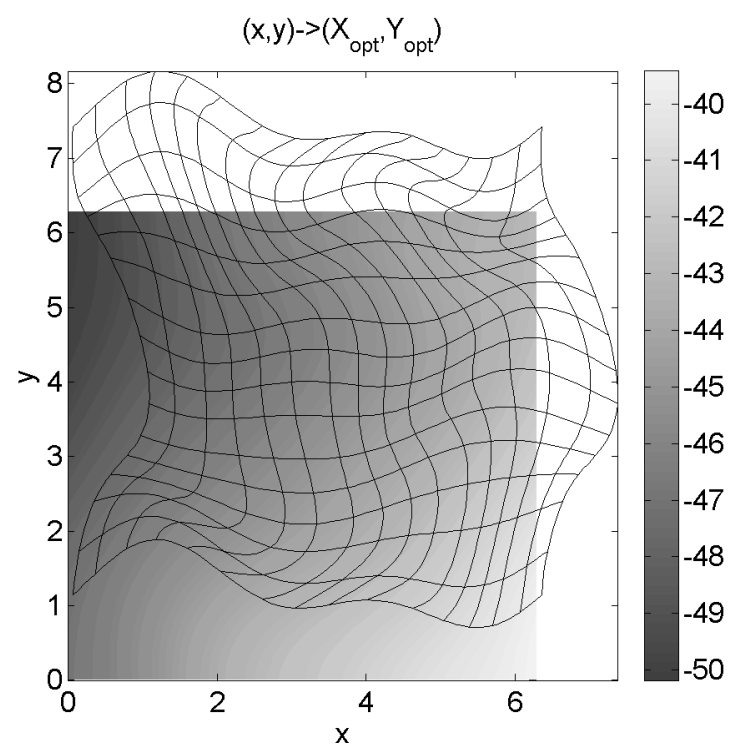

(b)

Figure 2: Position realignment (a) a forecast of a prominent feature with no background noise (upper-left), the true feature with amplitude perturbations (upper-right), an optimal realignment obtained by a standard constrained numerical optimization code (lower-left), and the residual between the optimization and the true feature (lower-right); (b) the displacement maps generated for the realignment as a mesh overlaying the corresponding map function $\zeta$, no regularization. 

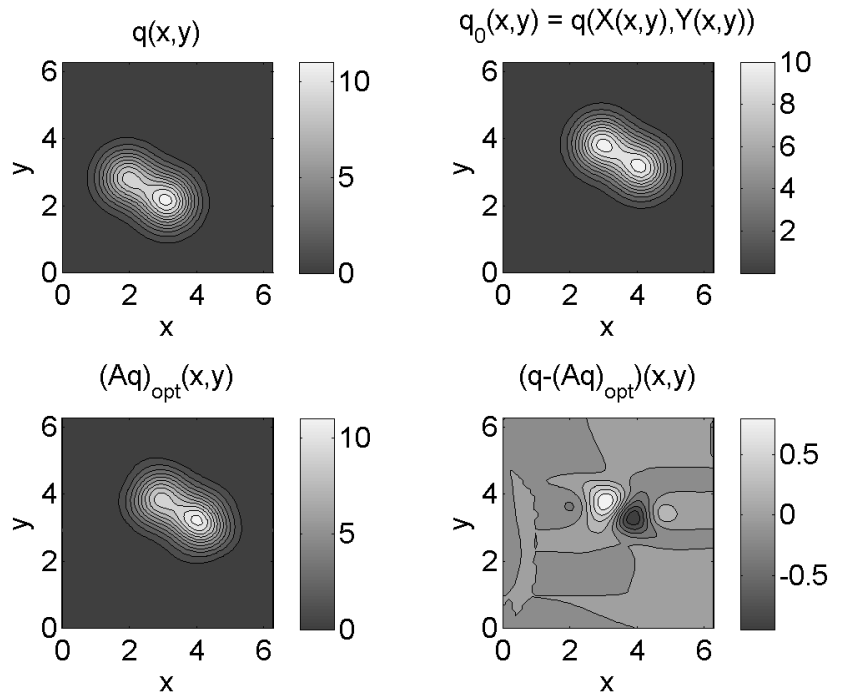

(a)

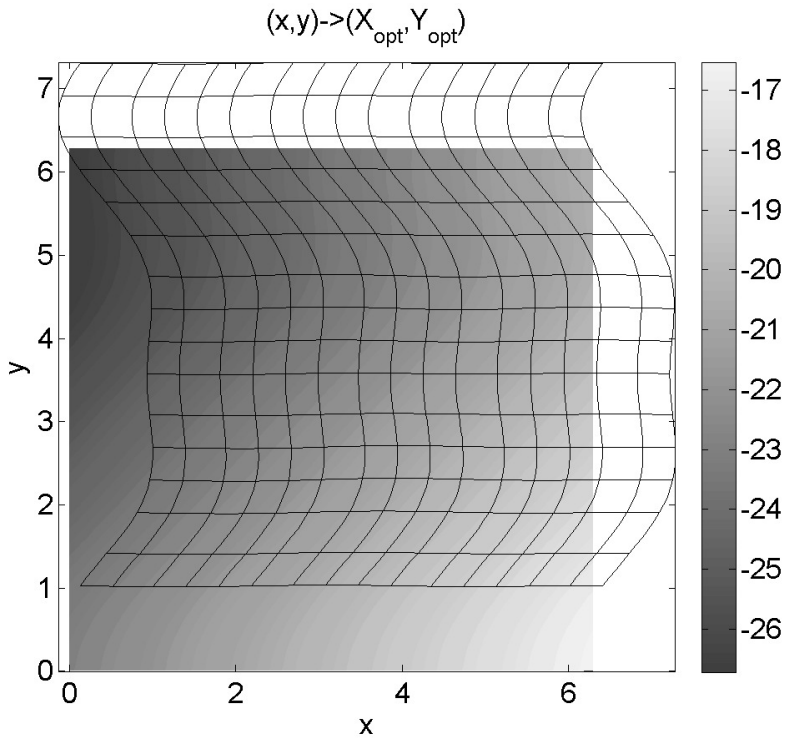

(b)

Figure 3: Position realignment, with strain regularization. Same panels as Figure 2, except that displacement maps have been regularized by the inclusion of the strain regularization term with $\alpha_{s}=10$ and $\alpha_{n}=0.1$. 
There is an issue here regarding how hidden regularizations imposed by the displacement map parameterization affect the model statistics. For any approximation method, the number of discretization points used determines the maximum roughness in the functions which can be modeled. For global methods, this is determined by the highest rank polynomial allowed, while for local methods like splines with fixed rank, the roughness is determined directly by the dimension of the subdomains. The variability of a function can be strongly determined by that of a random displacement map, and local spline interpolants provide a natural way to set the decorrelation length scale of its perturbations. Clearly, a calibration must be performed here to ensure that the map coefficients have the proper statistics for a given amplitude model error, though the details of such calculations do not appear in the published literature. We partially addressed this issue explicitly in Equation (18), and will take it up again after introducing the particular displacement map parameterization we will use.

We will adopt the bicubic spline representation, which has a convenient representation as a linear combination of B-splines [16. With the B-spline formulation rectangular approximations of complex boundaries are easily carried out. For any $p \in C^{2}(D)$, define a uniform rectangular partition of the domain with grid line intersections at nodes $\left\{\left(x_{j}, y_{j}\right)\right\}$ and spacings $\ell=\Delta x=\Delta y$, and extend this partition one layer of nodes into the exterior of the boundary. For example, a $3 \times 3$ array of sub-rectangles will have $4 \times 4=16$ nodes in $D$, and a ring of 20 nodes immediately outside the domain boundary, spaced exactly one grid spacing away. Then $p$ is exactly represented by the linear combination of tensor products of B-splines,

$$
p(x, y)=\sum_{j} a_{j} B_{j}(x, y)=\sum_{j} a_{j} b\left(x ; x_{j}\right) b\left(y ; y_{j}\right)
$$


where the B-spline kernel $b\left(z ; z_{j}\right)$ is the piecewise-cubic polynomial

$$
b\left(z ; z_{j}\right)=\left\{\begin{array}{cc}
\frac{1}{6} s^{3} & 0 \leq s<1 \\
\frac{1}{6}\left(-3 s^{3}+12 s^{2}-12 s+4\right) & 1 \leq s<2 \\
\frac{1}{6}\left(3 s^{3}-24 s^{2}+60 s-44\right) & 2 \leq s<3 \\
\frac{1}{6}\left(-s^{3}+12 s^{2}-48 s+64\right) & 3 \leq s \leq 4 \\
0 & \text { otherwise }
\end{array}\right.
$$

and $s=2+\left(z-z_{j}\right) / \ell$ is the local normalized coordinate parameterizing the support of the kernel. The extra ring of B-splines around the exterior provides the extra polynomial basis support on each sub-domain along the boundary to make the computation of polynomial coefficients there well-determined.

As a parameterization for the map function $\Psi$, bicubic B-splines like other linear approximation schemes allow us to conveniently represent the map function as a linear operation on the spline coefficients, $\Psi=B \cdot a$, as well as any linear operation on $\Psi$, such as the displacement map in Equation 15, or the local strains induced by the map in Equation 21). The position error penalty function in Equation 23 can be represented directly in terms of the spline basis, and amplitude covariance information is mapped onto position perturbation coordinates using the diagnostic relationship between the vorticity and stream functions, Equation (6). This resolves the issue of the degree of regularization imposed by the displacement function parameterization, since the position coefficient statistics will be computed directly from the amplitude covariance. For example, if $\delta \omega \sim \mathcal{N}\left(0, C_{\omega}\right)$, and we want to approximate position uncertainty by a Gaussian distribution, $\delta \Psi \sim \mathcal{N}\left(0, C_{a}\right.$, then we can estimate $C_{a}=T_{a \omega} C_{\omega} T_{a \omega}^{T}$.

For the remainder of this Section, we address a common issue encountered when computing statistical covariances in theoretical, numerical, and observational contexts. Techniques that estimate, propagate, or regularize statistical data often destroy the structure that distinguishes a valid covariance matrix from a generic square matrix. In these cases, one needs to (re)project the information back into the space of covariance matrices, i.e. symmetric positive semi-definite (SPSD) matrices. The sense in which a candidate projection is 
closest to the given matrix also must be determined. Consider a small perturbation to a SPSD matrix $A$ that contributes a negative eigenvalue: take any $u$ in its nullspace and add the rank-1 perturbation $-\epsilon u u^{T}$ for some $0<\epsilon \ll 1$. Then $A$ is no longer positive semi-definiteness. Then subsequent covariance operations, such as the Kalman filter covariance update, can yield unexpected results. While there are methods of finding the closest SPSD matrix in the 2-norm, they are much more theoretically and computationally complex than in the Frobenius norm. A polar factorization of the symmetric matrix component of $A$, given by $B=\left(A+A^{T}\right) / 2$, can be accomplished through a singular value decomposition. If $B=U_{B} \Sigma_{B} V_{B}^{T}$, where $U_{B}$ and $V_{B}$ are unitary and $\Sigma_{B}$ is diagonal and positive semi-definite, then

$$
B=Q H=\left(U_{B} V_{B}^{T}\right)\left(V_{B} \Sigma_{B} V_{B}^{T}\right)
$$

Observe that the first factor on the right-hand side is unitary, and since the second factor is a similarity transformation of $\Sigma_{B}$, then the result is SPSD.

Anticipating the needs of the two-stage statistical model, we also need an observation operator for the kind of displacement maps posed in 15 , or rather the map parameters which index them. These are of course the components, at the observed locations, of the image of the velocity function under the coordinate transformation $Z=\Phi(z ; a)$, for which we can write

$$
\begin{aligned}
& h_{p}\left(a ; \omega ;\left\{x_{d, j}, y_{d, j}\right\}\right)= \\
& h_{a}\left[\omega \circ \Phi^{-1}(\cdot ; a) ;\left\{x_{d, j}, y_{d, j}\right\}\right]
\end{aligned}
$$

Note that a continuous interpolation for the vorticity function $\omega$ must be used to estimate the velocity at mapped observation locations, for which the most accessible choice is the current forecast $\omega^{f}$, although other choices may be possible.

At this point, the only detail left to be addressed before an analysis scheme can be presented relates to the projection of forecast vorticity amplitude information into position coordinates. Such a method already has been introduced in Equation (18). The new wrinkle here is the imposition of boundary con- 
ditions, without which the transformation operator from map parameters to vorticity values, $T_{\omega a}$, will not have full rank. This would preclude the definition of its pseudoinverse $T_{a \omega}=T_{\omega a}^{\dagger}$, which is the desired projection operator. In other words, the space of displacement map parameters must be restricted to a dimension equal to the degrees of freedom unconstrained by the boundary conditions. There are three sets of conditions to be imposed on the coefficients of the map function $\Psi(z ; a)=\sum_{j=1}^{N_{c}} a_{j} B_{j}(z)$, where $N_{c}=\left(N_{c, x}+3\right) \cdot\left(N_{c, y}+3\right)$ is the number of B-spline coefficients: The Laplacian of the map function must be a fixed constant on $\partial D$, so we set $\Delta \Psi=0$ on these nodes. The tangent gradient of the map function must be zero on $\partial D$, so we set $\Psi_{y}=0$ for $x \in\left\{x_{L}, x_{U}\right\}$, and $\Psi_{x}=0$ for $y \in\left\{y_{L}, y_{U}\right\}$. The height of the map function must be fixed on $\partial D$, so we set $\Psi\left(x_{L}, y_{L}\right)=0$. The tangent condition prevents the map from moving vorticity mass out of the domain $D$ where it is lost to the model. Since the displacement map is defined by derivatives of the map function, any constant term disappears. Constants typically are the first term of any basis for $L^{2}$ function representations on a compact domain, including bicubic splines, and the height condition removes this degree of freedom. These linear conditions on the map parameters can be expressed by the linear system

$$
0=W \cdot a=\left(U_{W} \Sigma_{W} V_{W}^{T}\right) \cdot a
$$

where the last equality factors the constraint matrix into its singular value decomposition. If $r_{W}$ is the rank of the constraint matrix, then the columns of $V_{b}$, defined to be the last $N_{c}-r_{W}$ columns of $V_{W}$, are a basis for the null space of $W$. Now any set of boundary-constrained map parameters can be represented by $a=V_{b} \cdot a_{b}$, and $T_{\omega a_{b}}=T_{\omega a} V_{b}$ will have full rank. Then the transformation operator from vorticity model space to boundary-constrained map parameter space is given by

$$
T_{a \omega}=V_{b} T_{\omega a_{b}}^{\dagger}
$$

Given a forecast covariance for the vorticity amplitude perturbations, $P_{\omega}^{f}$, then a forecast displacement map parameter covariance, for perturbations subject to 
zero-outflow boundary conditions, can be estimated from the projection $P_{a}=$

\subsection{Two-stage Analysis Scheme}

At each assimilation time $t_{k}$ for $1 \leq k \leq N_{a}$ we seek a model (vorticity) state analysis $\omega=\omega^{a}$ which minimizes a likelihood functional that has been conditioned (or regularized) by a forecast penalty term,

$$
\begin{aligned}
\mathcal{J}_{a}[\omega] & =\frac{1}{2}\left\|d_{V}-h_{a}\left(\omega ;\left\{z_{d, j}\right\}\right)\right\|_{R^{-1}}^{2} \\
& +\frac{1}{2}\left\|\omega-\omega^{f}\right\|_{\left(P_{\omega}^{f}\right)^{-1}}^{2}
\end{aligned}
$$

where $d_{V}$ are observations of the velocity field at discrete points in $D$. Denote the time series of analysis ensembles in enKF by $\mathcal{E}_{k}^{a}=\left\{\omega_{j}^{a}\left(z, t_{k}\right)\right\}$, for $1 \leq j \leq N_{\text {ens }}$ and $0 \leq k \leq N_{a}$, initialized with the exact initial condition, $\omega_{j}\left(z, t_{0}\right)=\omega_{0}(z)$, for each ensemble member. See Appendix B. The goal of the enKF is to compute these analysis ensembles by statistical interpolation of forecast and observation ensembles at each analysis step. Denote this the amplitude analysis step, which specifically consists of the following: each forecast ensemble $\mathcal{E}_{k}^{f}$ is produced by integrating the generalized model over $t \in\left(t_{k-1}, t_{k}\right]$, using the previous analysis ensemble $\mathcal{E}_{k-1}^{a}$ as an initial condition. Each forecast member becomes the estimate of the forecast mean, $\omega^{f} \rightarrow \omega_{j}^{f}\left(\cdot, t_{k}\right)$, in their own copy of (30), with synthetic observations perturbed from original measurements,

$d_{V, k, j}=d_{V, k}+\epsilon_{d, j, k}$, with $\epsilon_{d, j, k} \sim \mathcal{N}(0, R)$, simulating the spread of the same likelihood distribution. The members of each analysis ensemble are correlated by taking the corresponding forecast covariance to be the sample covariance from the forecast ensemble,

$$
P_{\omega, k}^{f}=\frac{1}{N_{e n s}-1} \mathcal{E}_{k}^{f}\left(\mathcal{E}_{k}^{f}\right)^{T}
$$

The enKF estimates a Kalman gain matrix, $K_{k}$, for the entire ensemble such that the linear combination of the simulated forecast and observation ensembles,

$$
\mathcal{E}_{k}^{a}=\mathcal{E}_{k}^{f}+K_{k} \cdot\left[d_{V, k}-h_{a}\left(\mathcal{E}_{k}^{f} ;\left\{z_{d, j}\right\}\right)\right]
$$


has minimal analysis variance when $h_{a}$ is linear and $N_{\text {ens }} \rightarrow \infty$. This Kalman gain follows the standard formula, replacing the forecast covariance with the sample covariance of the forecast ensemble,

$$
K_{k}=P_{\omega, k}^{f} H_{a, k}^{T}\left(R+H_{a, k} P_{\omega, k} H_{a, k}^{T}\right)^{-1}
$$
increments are first projected into the much lower dimensional map parameter space. Forming the mean vorticity forecast ensemble $\hat{\mathcal{E}}^{f}$ from concatenated copies of the ensemble sample mean, then the forecast map parameter covariance 
can be written as

$$
\begin{aligned}
P_{a, k}^{f}= & \frac{1}{N_{e n s}-1}\left[T_{a \omega}\left(\mathcal{E}_{k, m}^{f}-\hat{\mathcal{E}}_{k, m}^{f}\right)\right] . \\
& {\left[T_{a \omega}\left(\mathcal{E}_{k, m}^{f}-\hat{\mathcal{E}}_{k, m}^{f}\right)\right]^{T} }
\end{aligned}
$$

and of course still is subject to regularization before it can be used in (34). The new index $m$ is defined below. There is no need to define a forecast map parameter ensemble, since it is 0 by construction. Let the analysis map parameter ensemble be denoted by $\mathcal{A}_{k, m}=\left\{a_{j, m}\left(t_{k}\right)\right\}$. Since the initial vorticity condition is known exactly, $\mathcal{A}_{0}=0$. At each subsequent analysis time $t_{k}$, the linear enKF position analysis update can be expressed as

$$
\begin{aligned}
\mathcal{A}_{k, m} & =0+L_{k, \alpha} \cdot\left[d_{V, k}-h_{p}\left(0 ; \mathcal{E}_{k, m}^{f},\left\{z_{d, j}\right\}\right)\right] \\
& =L_{k} \cdot\left[d_{V, k}-h_{a}\left(\mathcal{E}_{k, m}^{f} ;\left\{z_{d, j}\right\}\right)\right]
\end{aligned}
$$

where the Kalman gain matrix $L_{k, \alpha}$ for the map parameter estimator depends on the strain regularization parameters $\alpha$, and is given by

$$
L_{k, \alpha}=P_{a, \alpha, k} H_{p, k}^{T}\left(R+H_{p, k} P_{a, \alpha, k} H_{p, k}^{T}\right)^{-1}
$$

where the linearization of the map parameter observation operator, $H_{p, k}$, must be taken with respect to $a$ and not $\omega$. From the chain rule, the linearization about $\mathcal{A}_{k, m}=0$ is

$$
\begin{aligned}
H_{p, k} & =\nabla_{a} h_{p}\left(\mathcal{A}_{k, m} ; \mathcal{E}_{k, m}^{f},\left\{z_{d, j}\right\}\right) \\
& =\nabla_{a}\left[\mathcal{E}_{k, m}^{f} \circ \Phi^{-1}\left(\cdot ; \mathcal{A}_{k, m}\right)\right] \\
& =\nabla_{z} \mathcal{E}_{k, m}^{f} \cdot \nabla_{a} \Phi^{-1}\left(\cdot ; \mathcal{A}_{k, m}\right)
\end{aligned}
$$

Given the linearity and skew-symmetry of the stream function displacement map $\Phi$ defined in 15 , then the gradient of the map about $\mathcal{A}=0$ is

$$
\begin{aligned}
& \nabla_{a} \Phi^{-1}(\cdot ; \mathcal{A}) \mid(\mathcal{A}=0) \\
= & \nabla_{a} \Phi(\cdot ;-\mathcal{A}) \mid(\mathcal{A}=0) \\
= & {\left[\begin{array}{c}
B_{y}(\cdot) \\
-B_{x}(\cdot)
\end{array}\right] }
\end{aligned}
$$


regardless of the map parameters $\mathcal{A}$, where we recall that $B(\cdot)$ are the basis functions of the map function $\Psi$. The resulting map parameter analysis ensemble defines a new forecast ensemble, presumably with less position error near observed features,

$$
\mathcal{E}_{k, m+1}^{f}=\mathcal{E}_{k, m}^{f} \circ \Phi^{-1}\left(\cdot ; \mathcal{A}_{k, m}\right)
$$

where now can be seen that $m$ counts the number of times this position correction process is repeated, $1 \leq m \leq M$.

\section{Vortex Tracking Twin Experiment}

A twin experiment was used to measure forecast and analysis errors by comparing the vorticity trajectory predicted by the standard and the displacement enKF to a truth which, like the forecast ensembles, is a simulation of the stochastic vorticity model. Observations of the entire truth are not permitted to be used with the filter, only a grid of noisy velocity measurements that have been synthetically generated from the true vorticity trajectory. The analysis times were spaced far enough apart to begin to see performance differences between the standard and the displacement enKF. Constant-height contours at assimilation times are provided in Figures 5 and 6 to illustrate the effectiveness of each enKF in forecasting and tracking the truth. The number of ensemble members was $N_{\text {ens }}=5$. A more general argument comparing the efficiency and accuracy of each filter is made in Section 4.1. The contours show that the strain regularized displacement maps appear to generate tighter forecasts. The contours of the two-stage analyses preserve the circular feature geometry of the truth with much higher fidelity than the analyses provided by the standard filter. The loss of structure may be due to the bias in forecast mean and inflation of forecast uncertainty that can arise from a spatially dispersed set of predictions of the same strong feature, such as an ensemble of realizations of the same vortex. Details supporting this claim will be presented in Section 4.1. That most of the forecast displacement is corrected without these distortions in the two-stage 
445 displacement maps.

In addition to the preservation of features, the performance of the standard and displacement enKF will be evaluated and compared by quantifying the resulting distributions of forecast error, $\epsilon^{f}:=\omega^{t}-\omega^{f}$, and analysis error, $\epsilon^{a}:=$ $\omega^{t}-\omega^{a}$. The error bias, $\mathbb{E}(\epsilon)$, in the forecast and analysis of each filter is measured by comparing these to the true vorticity function at each analysis time in the $L_{2}$-norm. The error variance, $\operatorname{Var}(\epsilon)$, is measured by computing the sample variance of these errors over several repetitions of the twin experiment, and measuring this variance in the $L_{1}$-norm. These are taken to be functions on the entire model domain, $D$, and not just the observed subset, $D_{d} \subset D$; since the model interpolates the data, it is expected that the most accurate predictions will occur at observed locations. When the domain is only sparsely observed, the errors measured on $D_{d}$ are not indicative of the accuracy of predictions made on all of $D$. Figure 7 gives the time sequences of forecast and analysis error biases and variances for the standard and two-stage filters. More specifically, these are the average error bias and variance statistics for the 16 repetitions of the twin experiment with $N_{\text {ens }}=5$. The consistently increasing error statistics are due to the fact that the boundary conditions do not permit energy to leave the system, as the stochastic forcing in the vorticity model continually increases the model variance over time. It was shown in Figure 1 that the model uncertainty is dominated by position perturbations in the vortex positions, as opposed to amplitude perturbations. Likewise, the majority of forecast error removal is done through the position analysis. The standard filter does not define position error directly, so it cannot remove as much of the forecast variance with each analysis step. This is why the error bias and variance is consistently higher with the standard enKF than for the displacment enKF.

\subsection{Performance Comparison}

The twin experiment of the previous section was repeated several times for several different ensemble sizes, $N_{\text {ens }}$ (Table 1). Only the true vortex trajectory 

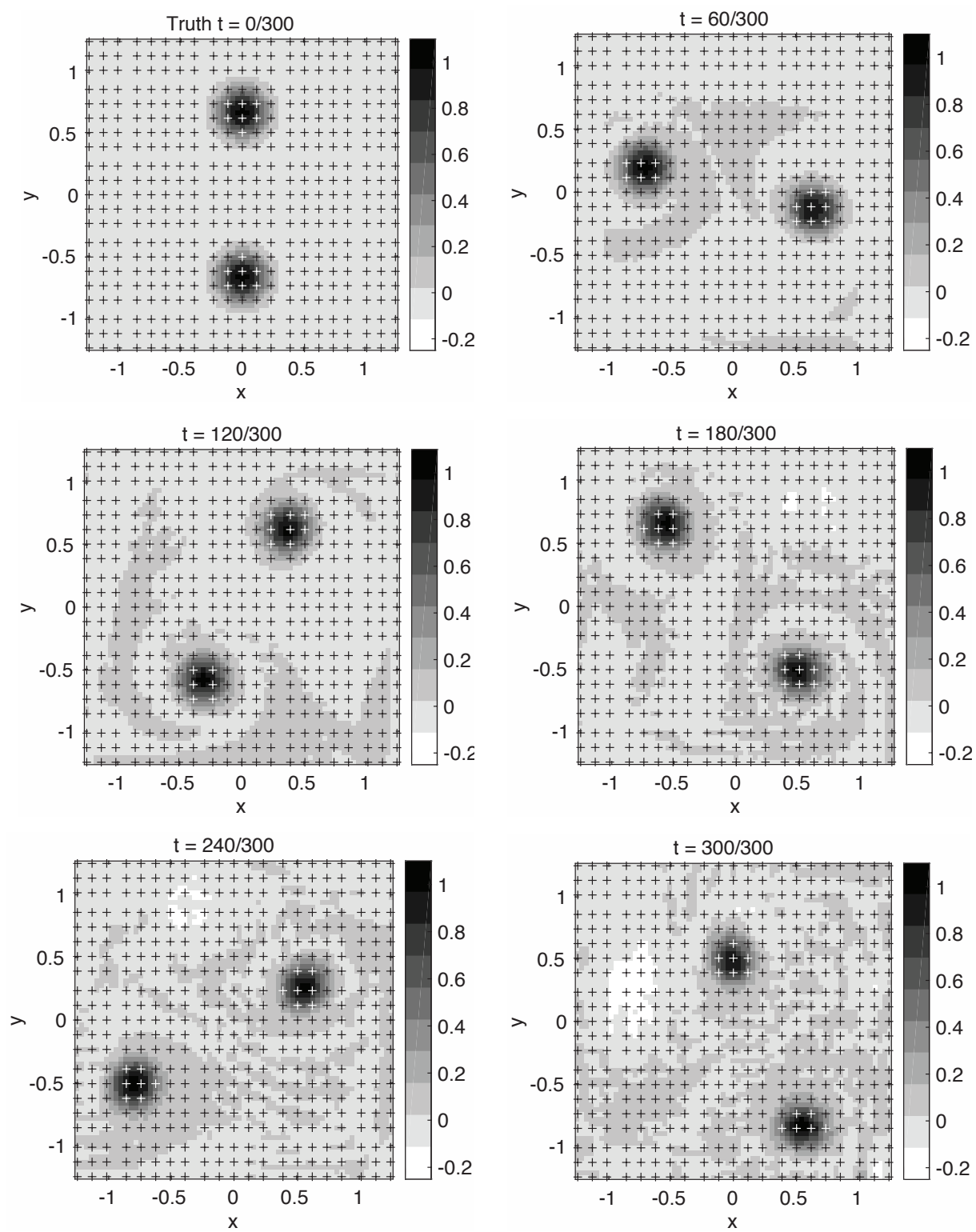

Figure 4: The common vorticity truth trajectory used in all twin experiments. The array of observation locations for each twin experiment is displaced as black "+s." Underlayed is a vorticity intensity map of one of the truth realizations, $\omega^{t}$. 

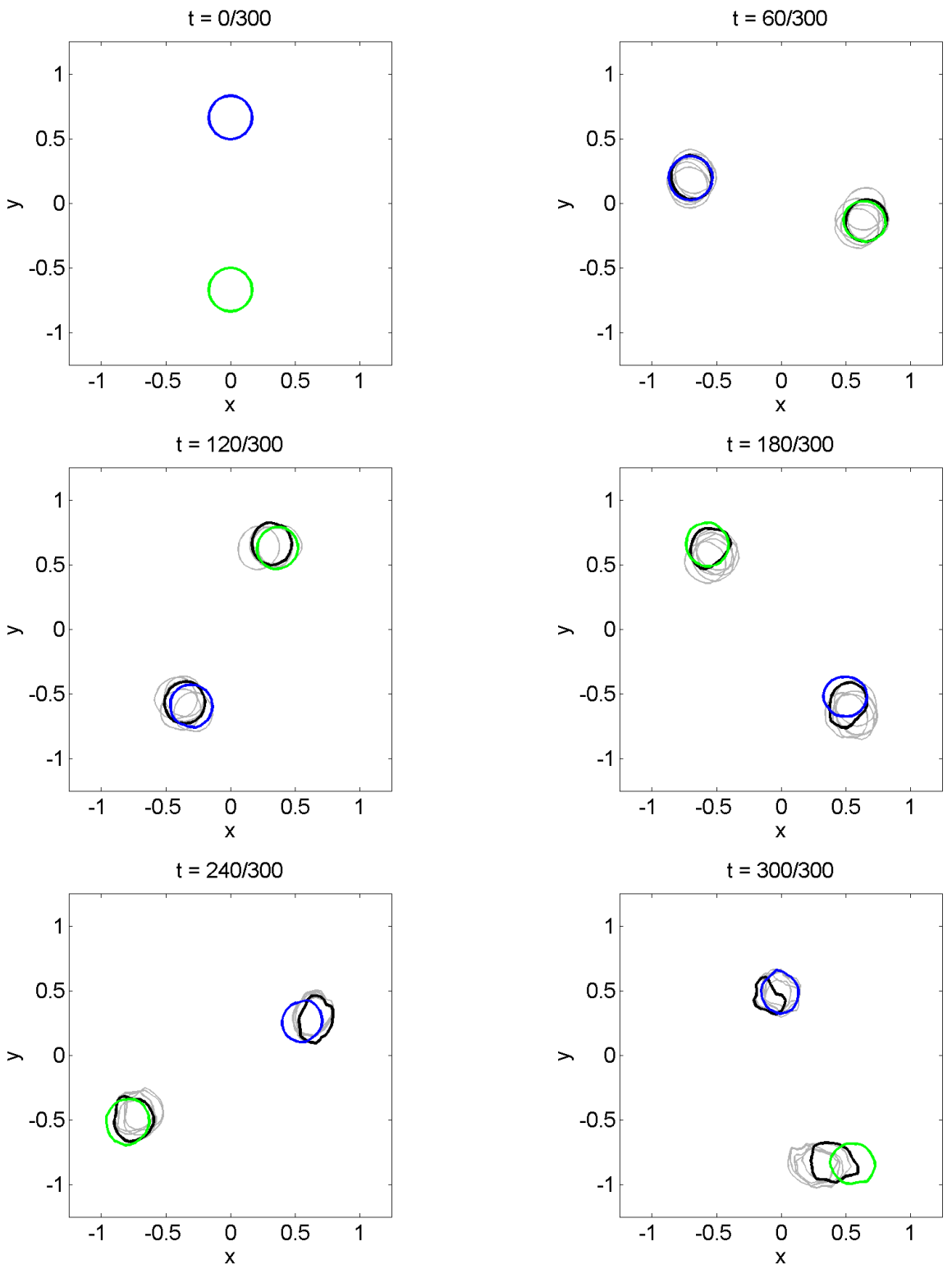

Figure 5: Standard enKF analysis of the true vortex positions in Figure 4, with $\mathbf{N}_{\text {ens }}=\mathbf{5}$. The forecast (gray), analysis (black), and truth (blue/green) vortices are depicted by equal-height contours at $\omega=.5$. 

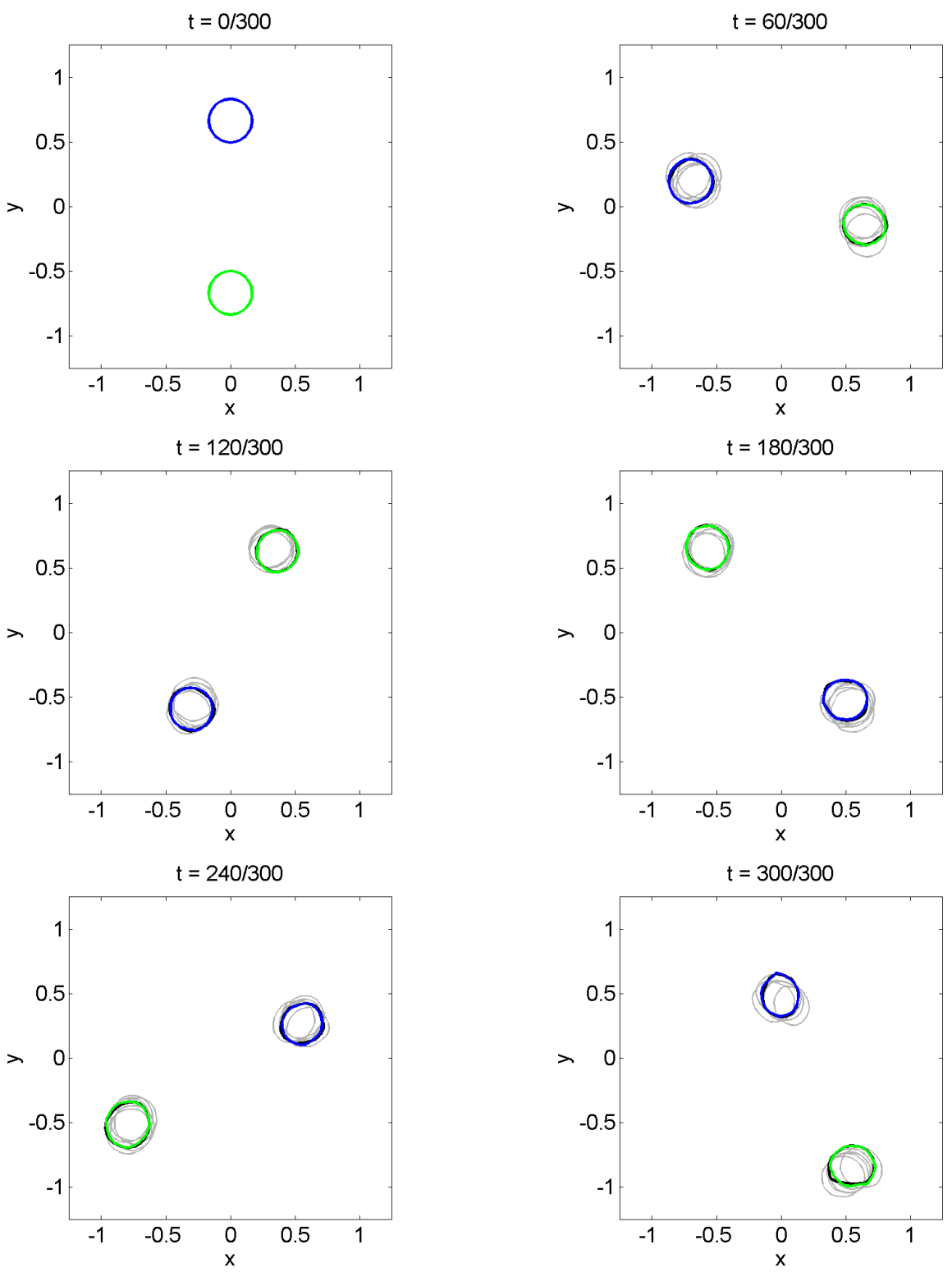

Figure 6: Displacement enKF analysis of the true vortex positions in Figure 4, with $\mathbf{N}_{\text {ens }}=\mathbf{5}$.. Same conditions as in Figure 5 

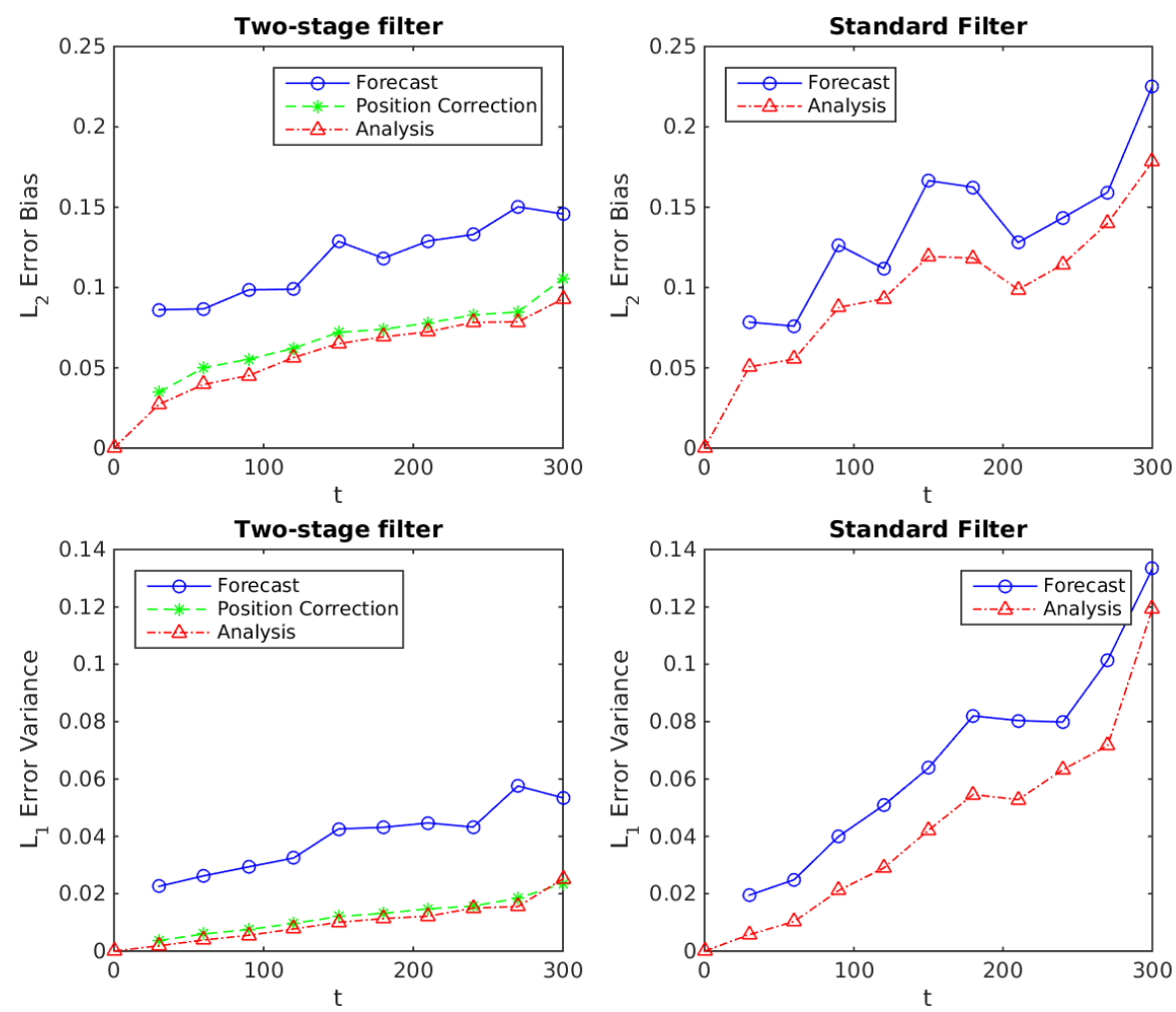

Figure 7: Forecast and analysis estimation errors in tracking co-rotating vortices with zero-outflow boundary conditions. 
Table 1: Repetitions of twin experiment with stochastic vorticity model.

\begin{tabular}{|l|cccc|}
\hline$N_{\text {ens }}$ & 5 & 10 & 20 & 40 \\
\hline Sample size & 16 & 12 & 10 & 8 \\
\hline
\end{tabular}

was was reused for each repetition, which facilitates a comparison of the performance of each enKF version, while controlling for variations in observation noise and ensemble selection. For each experiment repetition, the observation errors and synthetic observations, as well as the model perturbations during ensemble integration, are driven by independent random processes. This variation is removed by averaging the error bias and variance statistics of all trials with a given ensemble size. Sensitivity to observation noise is not performed here. Rather, since the model variance naturally increases with time, then an examination of the forecast and analysis errors at different model times, $t=150$ and 300, provides sensitivity information with respect to changes in a model variance parameter.

Figure 8 provides numerical evidence that both the standard and the displacement enKF estimates converge, although the displacement enKF does so more slowly. While the analysis bias error left by the standard enKF decays with order approximately $2 / 3$ and is robust to changes in model variance, the analysis bias of the displacement enKF is approximately half that, or order $1 / 3$, and slows by half again to order $1 / 6$ as model variance increases.

The forecast error bias indicates the predictive accuracy of the filter. It need not decay to zero, even if the analysis error does, since the accuracy of the prediction depends on more than the initial condition. Hence, a meaningful convergence rate is difficult to define or compute. However, a qualitative comparison of the forecast biases of the two filters shows the displacement filter is helping to improve predictive accuracy. The gains are nominal for low model variance, but as it increases, the forecast bias of the standard enKF increases for smaller ensembles $\left(N_{\text {ens }}<40\right)$, while the performance of the displacement enKF remains stable. This means the relative accuracy of the predictions of 

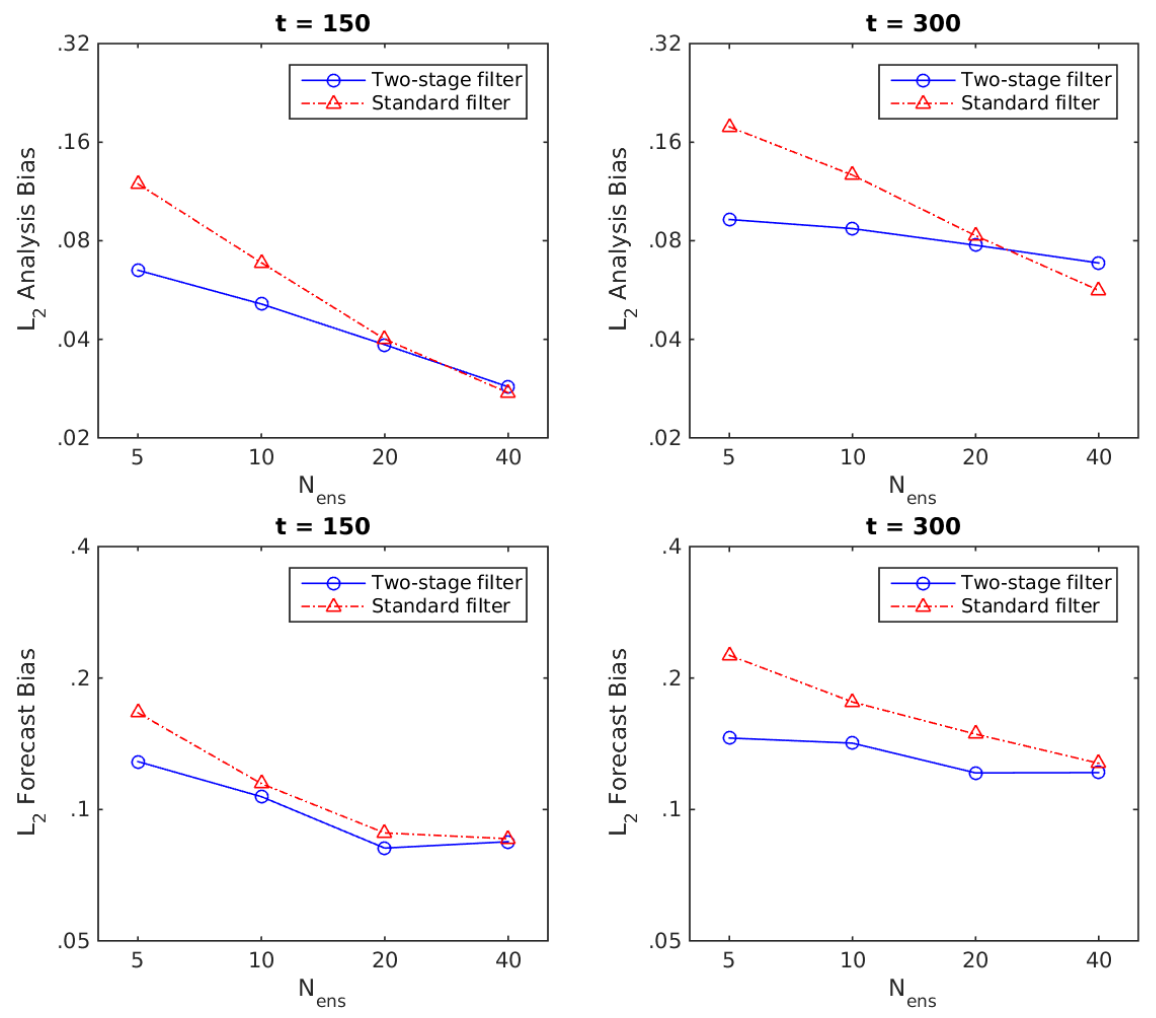

Figure 8: Decay of forecast and analysis error biases, $L_{2} \mathbb{E}(\epsilon)$, with increasing ensemble size.

the displacement filter improves over that of the standard filter as model error increases.

The forecast and analysis error variance measures the confidence one can have in the state estimates provided by the filter, before and after, respectively, the assimilation of new data. Measurements of the uncertainty in predictions are as important as the predictions themselves, and in these terms the quantitative evidence in support of using a forecast displacement preconditioner is positive (Figure 9). The analysis error variance of the standard enKF decays at a robust order $4 / 3$, and again for smaller ensembles the modified enKF is slower to converge. However, for medium-sized ensembles and possibly larger, the displacement filter appears to regain the convergence of the standard method, 
Table 2: Percentage reduction in forecast variance, measured in the $L_{1}$ norm, by using displacement enKF, at $t=150$.

\begin{tabular}{|ccccc|}
\hline$N_{\text {ens }}$ & 5 & 10 & 20 & 40 \\
\hline$L_{1}$ (standard) & .064 & .036 & .023 & .019 \\
\hline$L_{1}($ two-stage $)$ & .043 & .029 & .021 & .019 \\
\hline$\%$ reduction & $\mathbf{3 3}$ & $\mathbf{1 9}$ & $\mathbf{9}$ & $\mathbf{0}$ \\
\hline
\end{tabular}

so that for all values tested, the displacement enKF provides more accurate analyses.

The displacement enKF also provides greater certainty in its state predictions, based on an analysis of the improvements in forecast variance reduction (Figure 9p. For the small- to medium-sized ensembles tested, the forecast error variance from the displacement filter was equal to or reduced compared to that of the standard filter. As the ensemble size decreases, the variance reduction increases up to $33 \%$ of the standard filter forecast error variance (Table 2). The improvement in variance reduction is more pronounced as model variance increases over time from $t=150$ to $t=300$, in which the percentage decrease in forecast error variance ranges from $12 \%$ to $60 \%$ depending on the ensemble size. Another way of looking at this data is in terms of the computational effort that is saved using the displacement filter to achieve a prescribed level of forecast error variance (Tables 2 and 3 ). As the model variance increases, the percentage reduction in the required ensemble size moves from approximately $30 \%$ to about $60 \%$. Either measured in terms of forecast variance reduction or computational efficiency, the evidence demonstrates that displacement filter can improve vortex tracking.

\section{Conclusions}

We proposed a data assimilation strategy for the estimation of the mean and the variance of the posterior distribution of a time evolution state variable subject to observations. It introduces an extra stage in the traditional sequential 

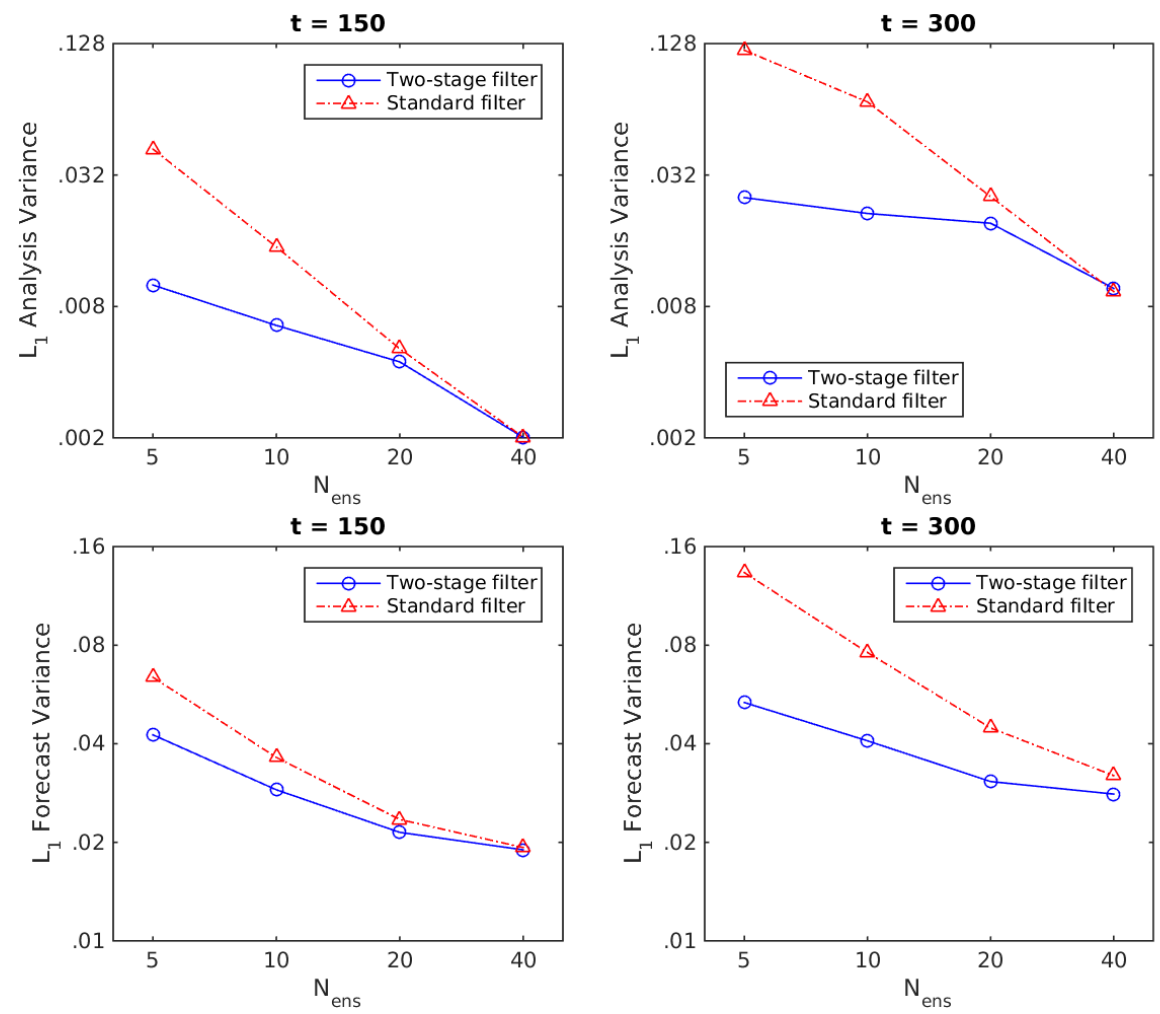

Figure 9: Decay of forecast and analysis error variance, $L_{1} \operatorname{Var}(\epsilon)$, with increasing ensemble size. 
Table 3: Percentage reduction in forecast variance, measured in the $L_{1}$ norm, by using a displacement enKF, at $t=300$.

\begin{tabular}{|ccccc|}
\hline$N_{\text {ens }}$ & 5 & 10 & 20 & 40 \\
\hline$L_{1}($ standard $)$ & .133 & .076 & .045 & .032 \\
\hline$L_{1}$ (two-stage) & .053 & .041 & .031 & .028 \\
\hline \% reduction & $\mathbf{6 0}$ & $\mathbf{4 6}$ & $\mathbf{3 1}$ & $\mathbf{1 2}$ \\
\hline
\end{tabular}

Table 4: Percentage computational savings by using the displacement enKF, at $t=150$.

\begin{tabular}{|cccc|}
\hline Desired $L_{1}$ forecast variance & .02 & .03 & .04 \\
\hline Required $N_{\text {ens }}$ (standard) & 35 & 14 & 9 \\
\hline Required $N_{\text {ens }}$ (two-stage) & 30 & 9 & 6 \\
\hline \% reduction in computation & $\mathbf{1 4}$ & $\mathbf{3 6}$ & $\mathbf{3 3}$ \\
\hline
\end{tabular}

data assimilation strategy, wherein a kinematically constrained transformation is applied in order to preserve geometric features of the state vector. Because the constraint is imposed as a mapping of physical space (and in evolution problems, by implication, on time) we denote the methodology as displacement assimilation. The addition of the constraint is not intimately tied to the Kalman Filter. However, we modified this particular estimation procedure to concretely suggest how the additional constraint is used to produce a displacement assimilation. The Kalman filter is one of the most widely used and familiar data assimilation schemes and thus comparisons of the traditional strategy and the displacement assimilation is instructive.

Morphing in data assimilation is not new, and in the image processing context, the assimilation constraints can be dictated by computational constraints, aesthetics, image processing constraints. Mariano in [15] and Frazin in [10] use morphing to counteract the smoothing effect of sharp features produced by assimilation procedures that minimize variance. Most Bayesian data assimilation methodologies aim to minimize the posterior covariance of the state given 
Table 5: Percentage computational savings by using the displacement enKF, at $t=300$.

\begin{tabular}{|cccc|}
\hline Desired $L_{1}$ forecast variance & .032 & .04 & .05 \\
\hline Required $N_{\text {ens }}$ (standard) & 40 & 25 & 18 \\
\hline Required $N_{\text {ens }}$ (two-stage) & 18 & 11 & 6 \\
\hline \% reduction in computation & $\mathbf{5 5}$ & $\mathbf{5 6}$ & $\mathbf{6 7}$ \\
\hline
\end{tabular}

observations, but there is no guarantee that these estimates are even physical. For generic nonlinear/non-Gaussian dynamics problems, data assimilation methodologies that are ad-hoc when applied to nonlinear/non-Gaussian dynamics problems, this can be a very important source of concern. Adding physical constraints on the estimation process presumably produces higher fidelity in the estimates. However, this is not necessarily what we are after here: the point of adding physical constraints and symmetries is to produce moments of the state that are based upon physically realizable samples or that obey essential basic laws implied by the symmetries. In evolution problems with high dimension state spaces, such as those common to geophysical fluid dynamics, the impact of the constraint can be significant since it is often the case that in these settings tend to high dimension state variables and more often than not, be very sparsely sampled and poorly observed and thus the likelihood is poorly sampled.

In incompressible fluid flow a central constraint is that the velocity be divergence free. This global constraint implies mass conservation. Locally, violations in the incompressibility lead to errors in the velocity itself and thus in the advection and dispersion of the fluid itself or the tracers it carries. This has a bearing on applications ranging from time dependent pollution dynamics, weather/climate, fluid mechanics. For example, pinpointing pollution sources and accurately tracking the advection of pollutants, or estimating wave characteristics, tracking large scale structures such as storm systems, etc. In twodimensional flows, which is the setting of the dynamics in the current work, we chose for the proof of concept of the method, area-preserving maps that 
automatically satisfy the conservation required by incompressibility in the flow.

The manner in which we invoke the extra constraints is not done here via a 


\section{Acknowledgements}

This research was made possible by a grant from The Gulf of Mexico Research Initiative, and by NSF DMS grant 0304890, and NSF OCE grant 1434198. Some of the research on this project was undertaken when JMR was a participant in Stockholm University's Rossby Faculty Fellowship program.

\section{Appendix A. Canonical Transformations in the Small-displacement Limit}

Canonical transformations are coordinate transformations with the useful property that a simply-connected area remains simply-connected and has the same area after the transformation. The derivation of their form and properties is technical but well discussed standard texts on classical mechanics (e.g. [17]), so only their form is introduced here. The displacement vectors $(x, y) \rightarrow(X, Y)$ for each coordinate are determined by a twice continuously differentiable map function $\zeta(X, y ; a)$, where $a$ are parameters which uniquely determine each map function. Then the coordinate transformation is given by the following implicit system of differential equations:

$$
\begin{aligned}
x & =X+\frac{\partial}{\partial y} \zeta(X, y ; a) \\
Y & =y+\frac{\partial}{\partial X} \zeta(X, y ; a)
\end{aligned}
$$

It can be readily confirmed that the coordinate transformation defined by this system is area preserving by showing that its Jacobian and that of its inverse is unity.

If we solve for the displacements $X-x$ and $Y-y$ in Equation A.1, we recover a system which approximately defines $\zeta$ to be a stream function for a velocity field parallel to the spatial increments. In the limit of small displacements, canonical transformations are equivalent to a particular divergence-free flow along stream function contours. The transformation equations can be rewritten so that the map $\Phi$ is an approximate cross-section of the flow. For a small time 
parameter $0<\epsilon \sim\|\nabla \zeta\| \ll 1$, define $\zeta_{\epsilon}(X, y)=\epsilon \Psi(x, y)$. Then,

$$
\begin{aligned}
& \frac{\delta x}{\delta t}=\frac{X-x}{\epsilon}=-\frac{1}{\epsilon} \frac{\partial \zeta_{\epsilon}}{\partial y}(X, y) \\
& \frac{\delta y}{\delta t}=\frac{Y-y}{\epsilon}=\frac{1}{\epsilon} \frac{\partial \zeta_{\epsilon}}{\partial X}(X, y)
\end{aligned}
$$

As $\epsilon \rightarrow 0$ (a formal limit) we get

$$
\begin{aligned}
& \frac{\delta x}{\delta t}=-\frac{\partial}{\partial y} \Psi(x, y) \\
& \frac{\delta y}{\delta t}=\frac{\partial}{\partial x} \Psi(x, y) .
\end{aligned}
$$

620 to the stream function definition for a conservative velocity field.

\section{Appendix B. The Ensemble Kalman Filter}

The ensemble Kalman Filter (enKF) [18, 2, is an ensemble-based data assimilation technique for sequential problems. As in the standard Kalman Filter, the filter completes the assimilation process in two stages: a forecast and an analysis stage. Unlike the linearized Kalman Filter, the extended Kalman Filter (EKF) (see 11 for background on the KF and EKF). The enKF uses an ensemble of model runs to compute a mean proposal at each filtering step. The analysis step is the same as in the Kalman Filter case. Namely, a Gaussian approximation is made of the local posterior density, of the state vector given observations. With this assumption it is possible to write down the update on the mean, given observations, and an estimate of the posterior covariance. In the process the ensemble (model) mean, and a sample approximation of the (model) covariance are used in calculating the update and the Kalman gain. The enKF is particularly attractive because it is easy to code and requires minimal modifications to existing codes representing models.

At time $t_{0}$, the random perturbations of initial conditions are $y_{j}^{a}\left(t_{0}\right) \sim p\left(y_{0}\right)$ for each $1 \leq j \leq N_{\text {ens }}$. Expressing ensembles as matrices of concatenated ensemble members, so that each analysis ensemble for $0 \leq k \leq N$ and forecast 
ensemble for $1 \leq k \leq N$ can be written as the matrices

$$
\mathcal{E}_{k}^{a}=\left[y_{1}^{a}\left(t_{k}\right)|\ldots| y_{N_{\text {ens }}}^{a}\left(t_{k}\right)\right] \quad \mathcal{E}_{k}^{f}=\left[y_{1}^{f}\left(t_{k}\right)|\ldots| y_{N_{\text {ens }}}^{f}\left(t_{k}\right)\right]
$$

Then each forecast ensemble $\mathcal{E}_{k}^{f}$ at time $t_{k}$ is just the column-wise integration of the model equations, using the previous analysis ensemble $\mathcal{E}_{k-1}^{a}$ at time $t_{k-1}$ as initial conditions. We will also represent the sample mean of an ensemble as

$$
\hat{\mathcal{E}}_{k}^{f}=\left[\hat{y}_{k}^{f}|\ldots| \hat{y}_{k}^{f}\right],
$$

where $\hat{y}_{k}=N_{e n s}^{-1} \sum_{j=1}^{N_{e n s}} y_{j}\left(t_{k}\right)$. The ensemble forecast covariance is

$$
P_{k}^{f}=\frac{1}{N_{e n s}-1}\left(\mathcal{E}_{k}^{f}-\hat{\mathcal{E}}_{k}^{f}\right)\left(\mathcal{E}_{k}^{f}-\hat{\mathcal{E}}_{k}^{f}\right)^{T} .
$$

Let the ensemble of synthetic observation realizations be denoted by $\mathcal{D}_{k}=$ $\left[d_{k}+\epsilon_{k, 1}|\ldots| d_{k}+\epsilon_{k, N_{e n s}}\right]$, where each normal variate $\epsilon_{k, j} \sim \mathcal{N}(I, \mathcal{R})$. The ensemble analysis and the forecast are related to each other via the standard Kalman filter estimator: The linear Kalman update for the analysis ensemble is given by

$$
\mathcal{E}_{k}^{a}=\mathcal{E}_{k}^{f}+K_{k} \cdot\left[\mathcal{D}_{k}-h\left(\mathcal{E}_{k}^{f}\right)\right]
$$

where the observation operator $h(\cdot)$ is applied column-wise to each of the forecast ensemble members. The shared Kalman gain matrix can be expressed as

$$
K_{k}=P_{k}^{f} H_{k}^{T}\left(H_{k} P_{k}^{f} H_{k}^{T}+R\right)^{-1}
$$

and $H_{k}=\nabla_{y} h(\mathcal{E}) \mid\left(\mathcal{E}=\mathcal{E}_{k}^{f}\right)$ denotes the column-wise gradient of the ensemble observation operator evaluated at each forecast ensemble member.

When the observations are sparse so that $N_{d} \ll N$, a more efficient variant of B.2, in terms of the observed forecast ensemble $h\left(\mathcal{E}_{k}^{f}\right) \in \Omega_{d}$, is used:

$$
K_{k}=\frac{\mathcal{E}_{k}^{f} h\left(\mathcal{E}_{k}^{f}\right)^{T}}{N_{\text {ens }}-1}\left[\frac{h\left(\mathcal{E}_{k}^{f}\right) h\left(\mathcal{E}_{k}^{f}\right)^{T}}{N_{\text {ens }}-1}+R\right]^{-1}
$$

One still can use the linearized observation matrix, $h\left(\mathcal{E}_{k}^{f}\right) \equiv H_{k} \mathcal{E}_{k}^{f}$, and achieve the same gain in efficiency. With this representation of the enKF analysis in (B.1), it can be written in as a weighted combination of the ensemble members,

$$
\mathcal{E}_{k}^{a}=\mathcal{E}_{k}^{f} \cdot W_{k}\left[\mathcal{D}_{k} ; h\left(\mathcal{E}_{k}^{f}\right), R\right] .
$$


Now, the weights $W_{k}$, rather than being dependent on the likelihood functional, are dependent on the Gaussian parameters of the likelihood distribution.

\section{Appendix C. Computational Parameters}

Parameters of the generalized vorticity model and parameters of the twostage filter:

Domain geometry: $D=[-1.25,1.25]^{2}$

Domain discretization: $N_{x}=N_{y}=64(N=4225)$

Initial condition parameters:

Vortex centroids $x: x_{c, 1}=x_{c, 2}=0$

Vortex centroids $y: y_{c, 1}=-y_{c, 2}=2 / 3$

Vortex radii: $r_{s, 1}=r_{s, 2}=1 / 3$

Vortex amplitudes: $a_{1}=a_{2}=1$

Time integration interval: $t \in[0,300]$

Integration time step: $\Delta t=0.05$

Model error boundary dampening width: $r_{b}=.1$

Position analysis iterations: $M=3$

Map parameter discretization: $N_{c, x}=N_{c, y}=20\left(N_{c}=441\right)$

Regularization parameters: $\alpha_{n}=\alpha_{s}=50$

Observation dimensions: $N_{d, x}=N_{d, y}=20\left(N_{d}=441\right)$

Observation error standard deviation: $\tau=10^{-3}$

Model error pointwise standard deviation: $\sigma_{V}=10^{-3}$

Model error decorrelation length: $r_{V}=0.707$

Model error mode eigenvalue tolerance: $\lambda\left(Q_{V}\right) \geq 10^{-14}$

Model error boundary dampening width: $r_{b}=.1$

Assimilation time step: $\Delta t_{a}=30$

\section{References}

[1] A. H. Jazwinski, Stochastic processes and filtering theory, Vol. 63, Academic Press, 1970. 
[2] G. Evensen, Data Assimilation: The Ensemble Kalman Filter, 2nd Edition, Springer, 2009.

[3] C. Wunsch, The Ocean Circulation Inverse Problem, Cambridge University Press, Cambridge, UK, 1996.

[4] R. N. Hoffman et. al., Distortion representation of forecast errors, Monthly Weather Review 123 (1995) 2758-2770.

[5] K. A. Brewster, Phase-correcting data assimilation and application to storm-scale weather prediction, Ph.D. thesis, University of Oklahoma (1999).

[6] K. A. Brewster, Phase-correcting data assimilation and application to storm-scale weather prediction. part i: Method description and simulation testing, Monthly Weather Review 131 (2002) 480-492.

[7] S. Ravela, K. Emanuel, D. McLaughlin, Data assmilation by field alignment, Physica D 230 (2007) 127-145.

[8] T. Nehrkorn, B. Woods, R. N. Hoggman, T. Auligne, Correcting for position errors in variational data assimilation, Monthly Weather Review 143 (2015) 1368-1381.

[9] J. R. Percival, Displacement assimilation for ocean models, Ph.D. thesis, University of Reading (2008).

[10] R. A. Frazin, Coronal mass ejection reconstruction from three viewpoints via simulation morphing. i. theory and examples, The Astrophysical Journal 24 (2012) 761-768.

670

[11] S. Ravela, Quantifying uncertainty of coherent structures, Procedia Computer Science 9 (2012) 1187-1196.

[12] I. Christiansen, Numerical simulation of hydrodynamics by the method of point vortices, Journal of Computational Physics 13 (3) (1973) 363-379. 
[13] P. Kloeden, E. Platen, Numerical Solution of Stochastic Differential Equations, Springer-Verlag, Berlin, 1992.

[14] S. C. Venkataramani, T. M. Antonsen, E. Ott, Anomalous diffusion in bounded temporally irregular flows, Physica D: Nonlinear Phenomena 112 (3) (1998) 412-440.

[15] A. J. Mariano, Contour analysis: A new approach for melding geophysical fields, Journal of Atmospheric and Oceanic Technology 7 (1990) 285-295.

[16] C. de Boor, Bicubic spline interpolation, Journal of Mathematics and Physics 41 (1962) 212-218.

[17] H. Goldstein, C. Poole, J. Safko, Classical Mechanics', 3rd Edition, Pearson Education, 2002.

[18] G. Evensen, Using the extended Kalman filter with a multilayer quasigeostrophic ocean model, Journal of Geophysical Research 97 (1992) 1790517924. 

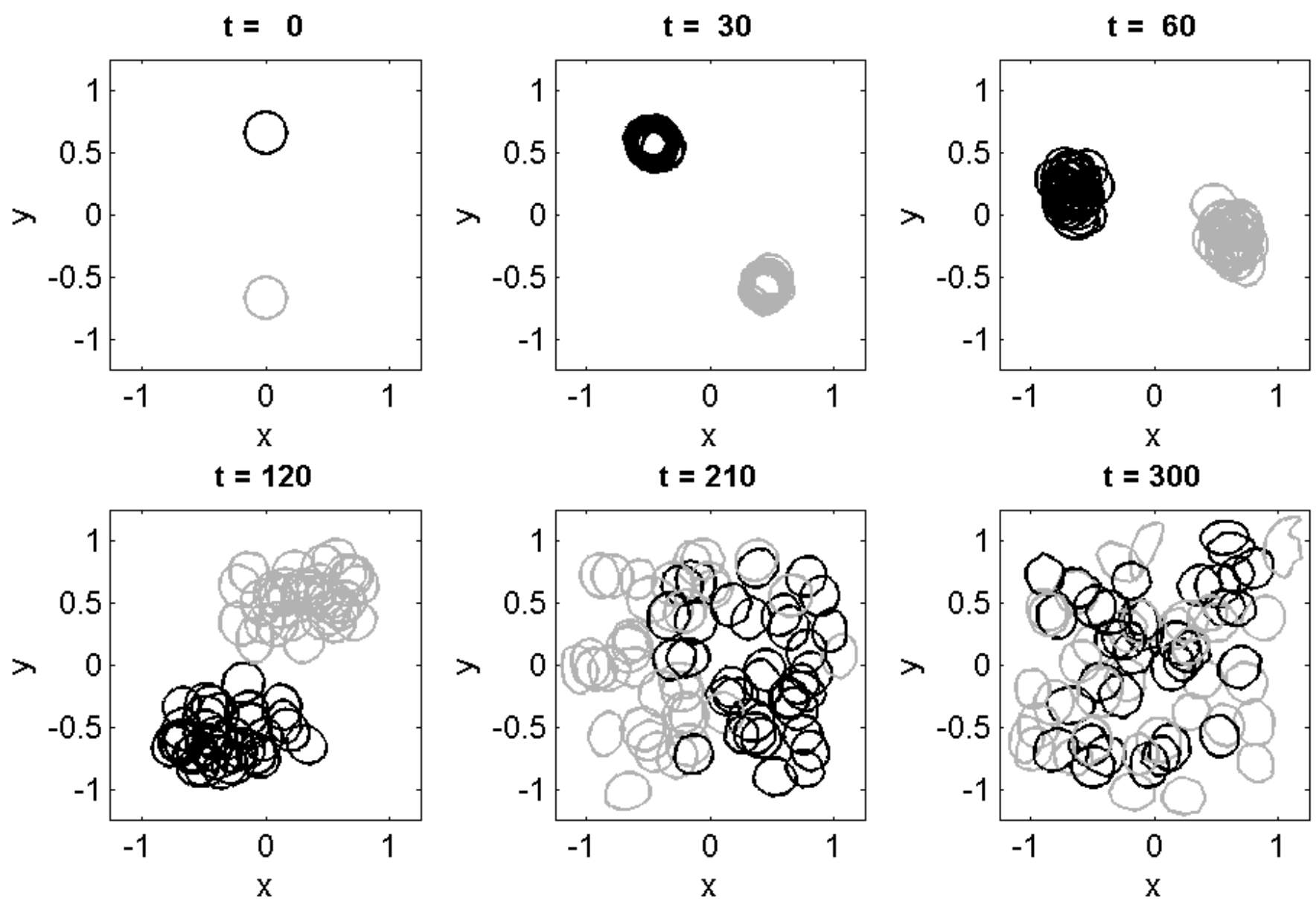

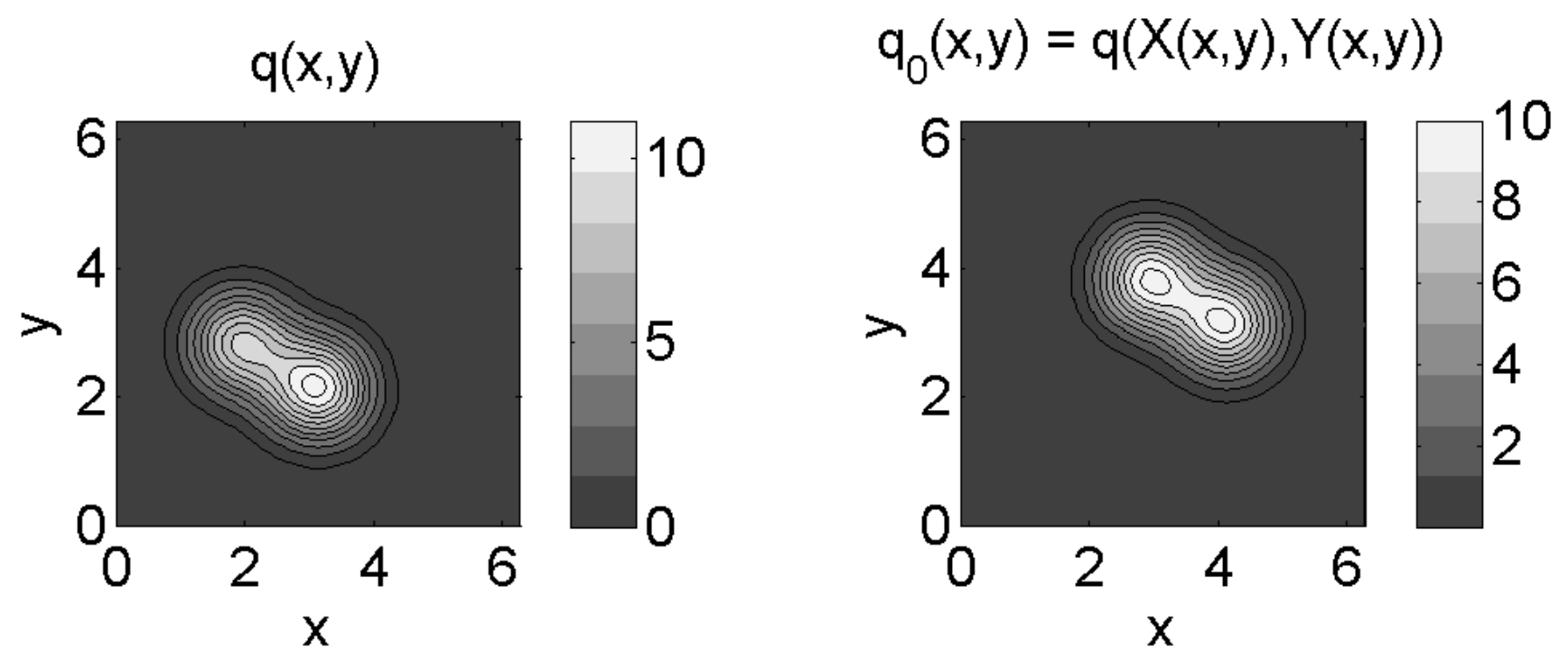

$$
(A q)_{\text {opt }}(x, y)
$$

$$
\left(q_{0}-(A q)_{o p t}\right)(x, y)
$$
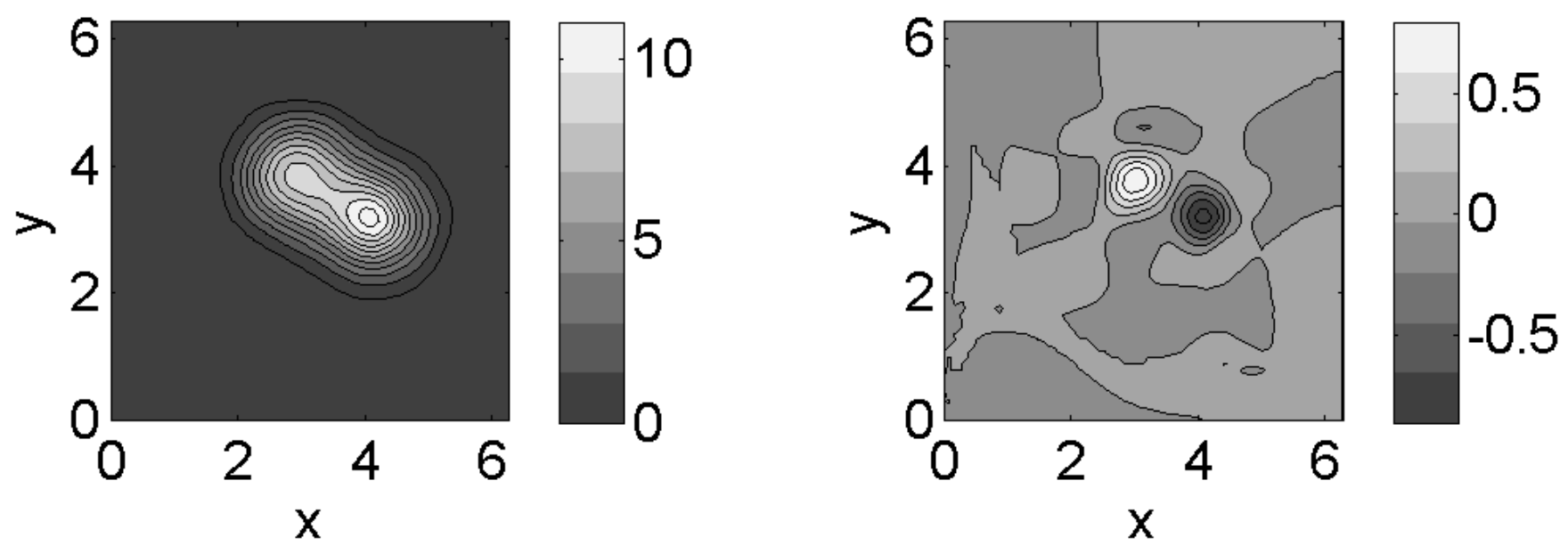


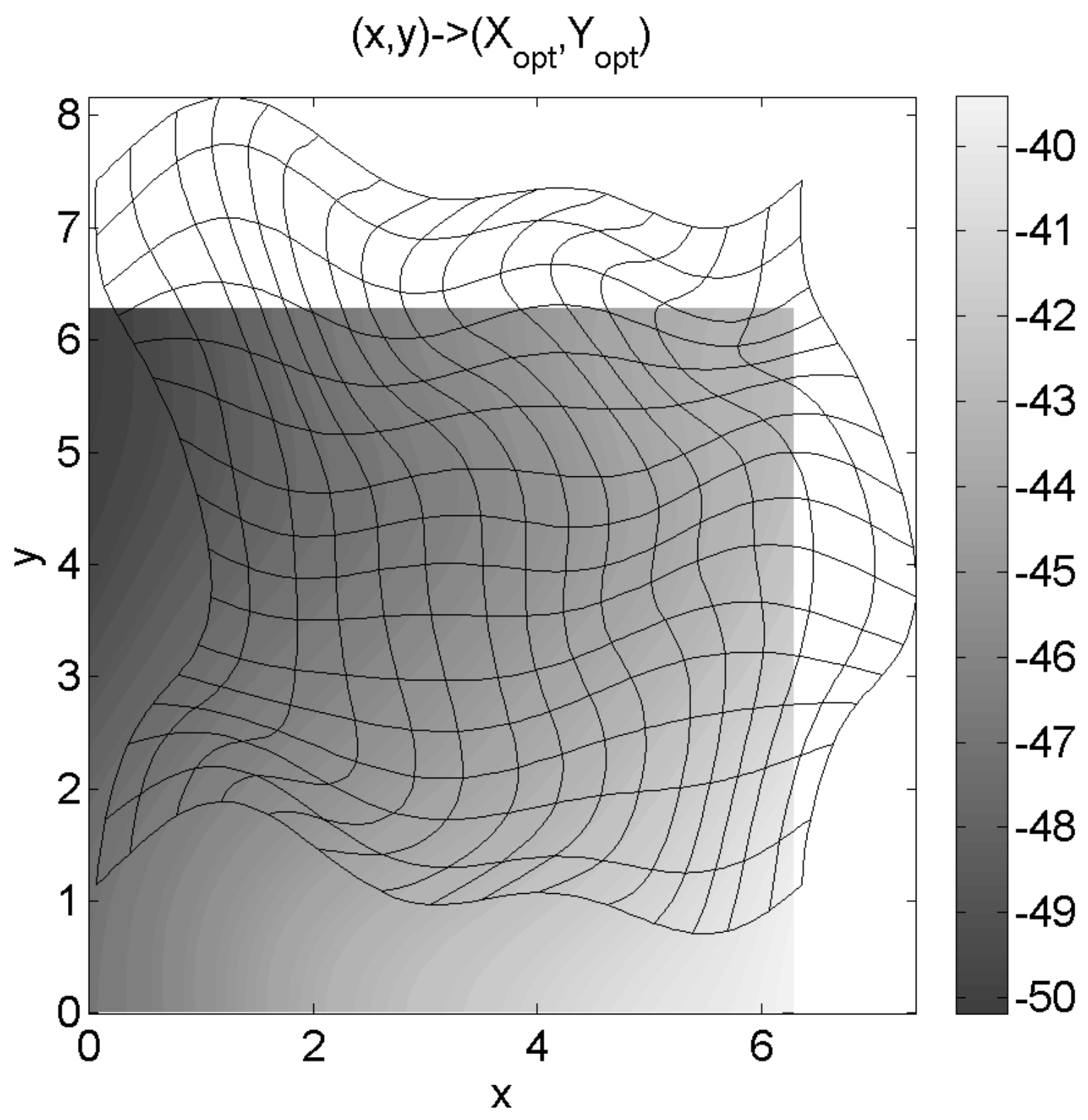



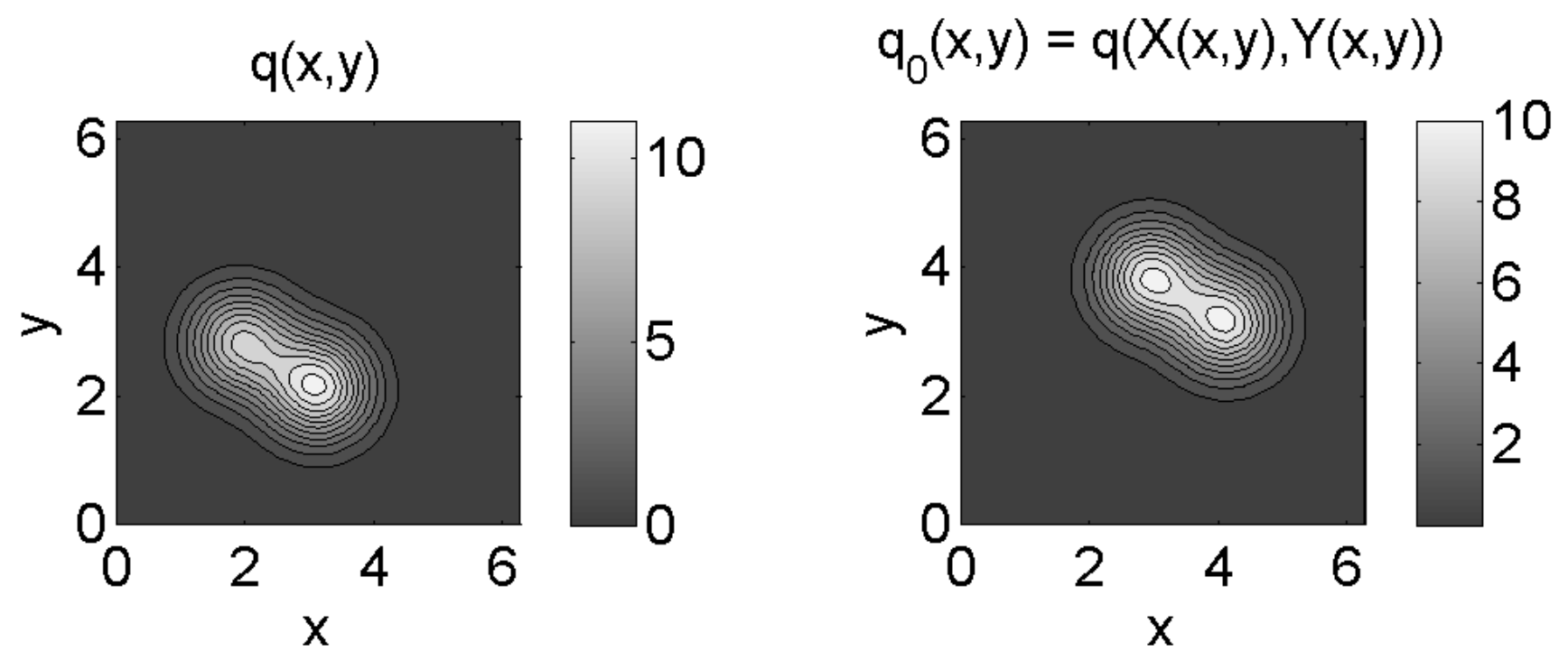

$$
(A q)_{o p t}(x, y)
$$

$$
\left(q-(A q)_{\text {opt }}\right)(x, y)
$$
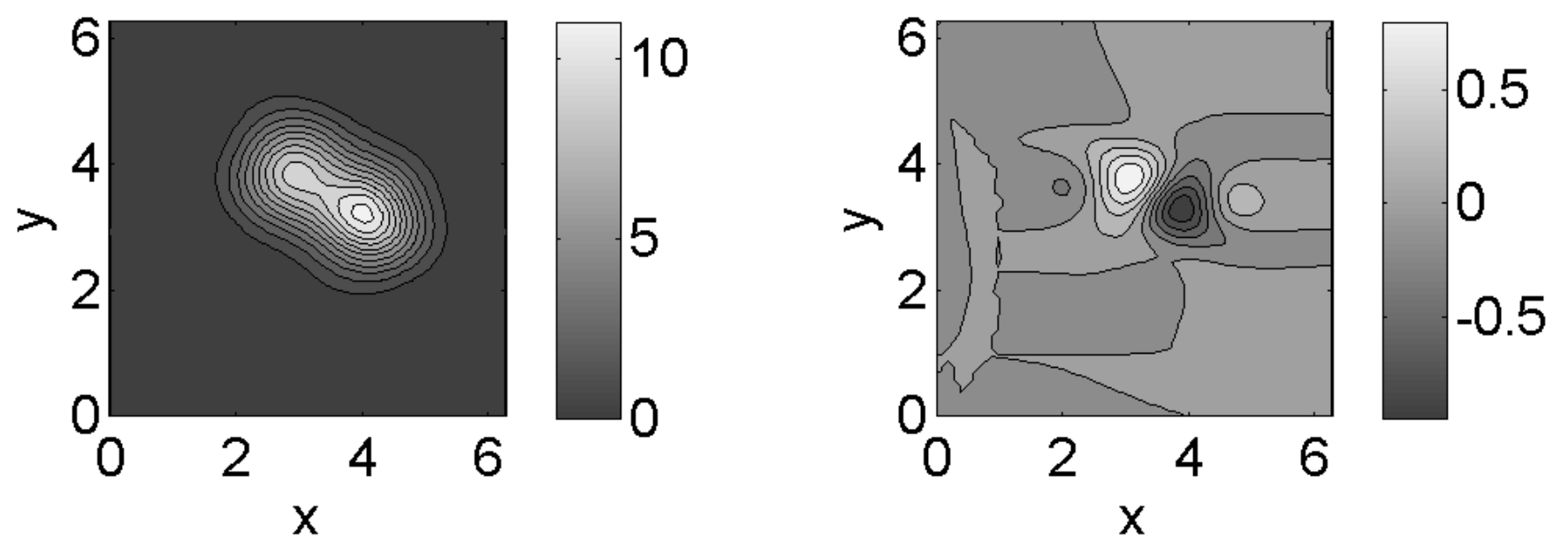


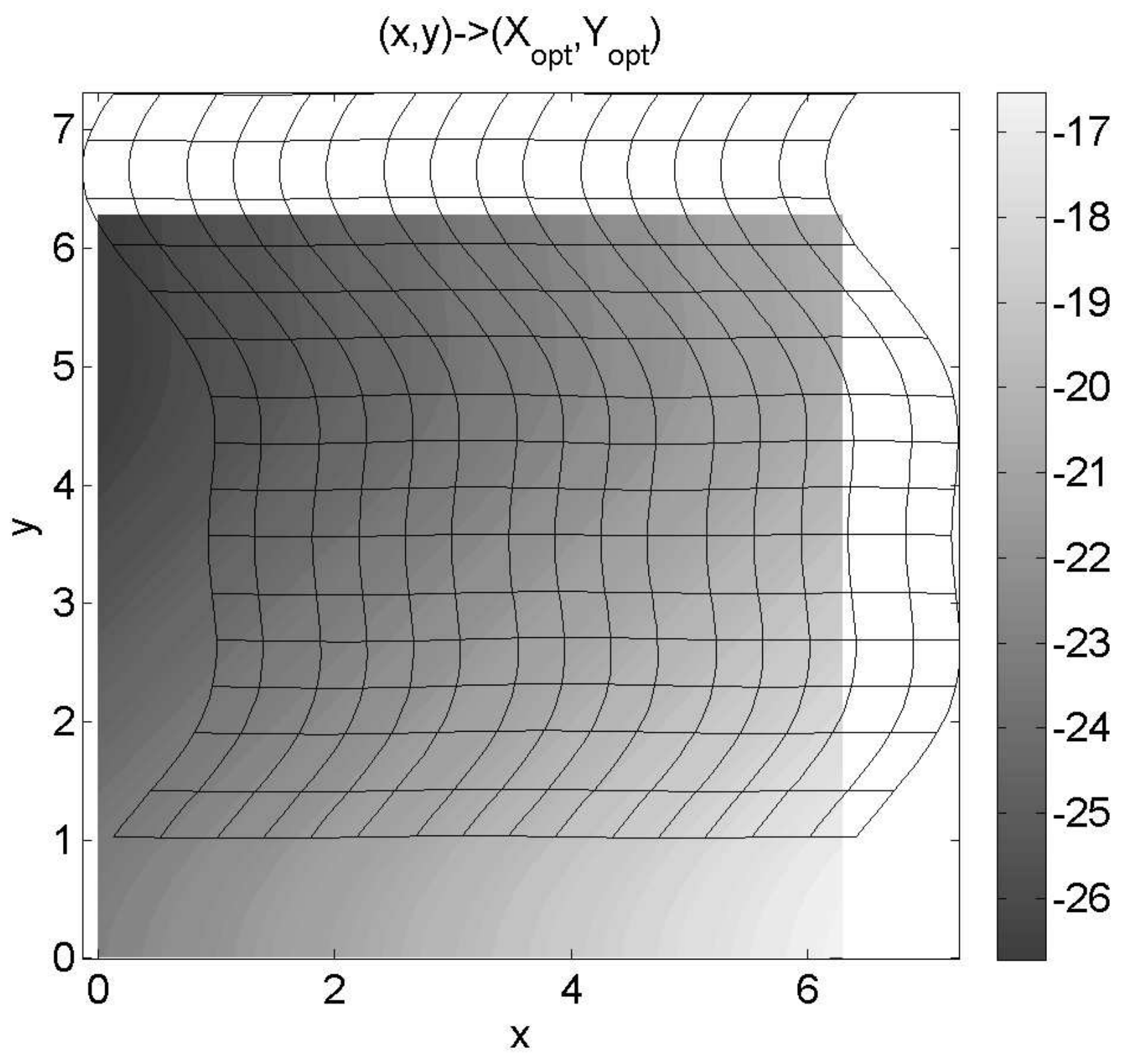

$-24$

25

$-26$

$(x, y) \rightarrow\left(X_{o p t} Y_{o p t}\right)$ 


\section{Truth $\mathrm{t}=0 / 300$}

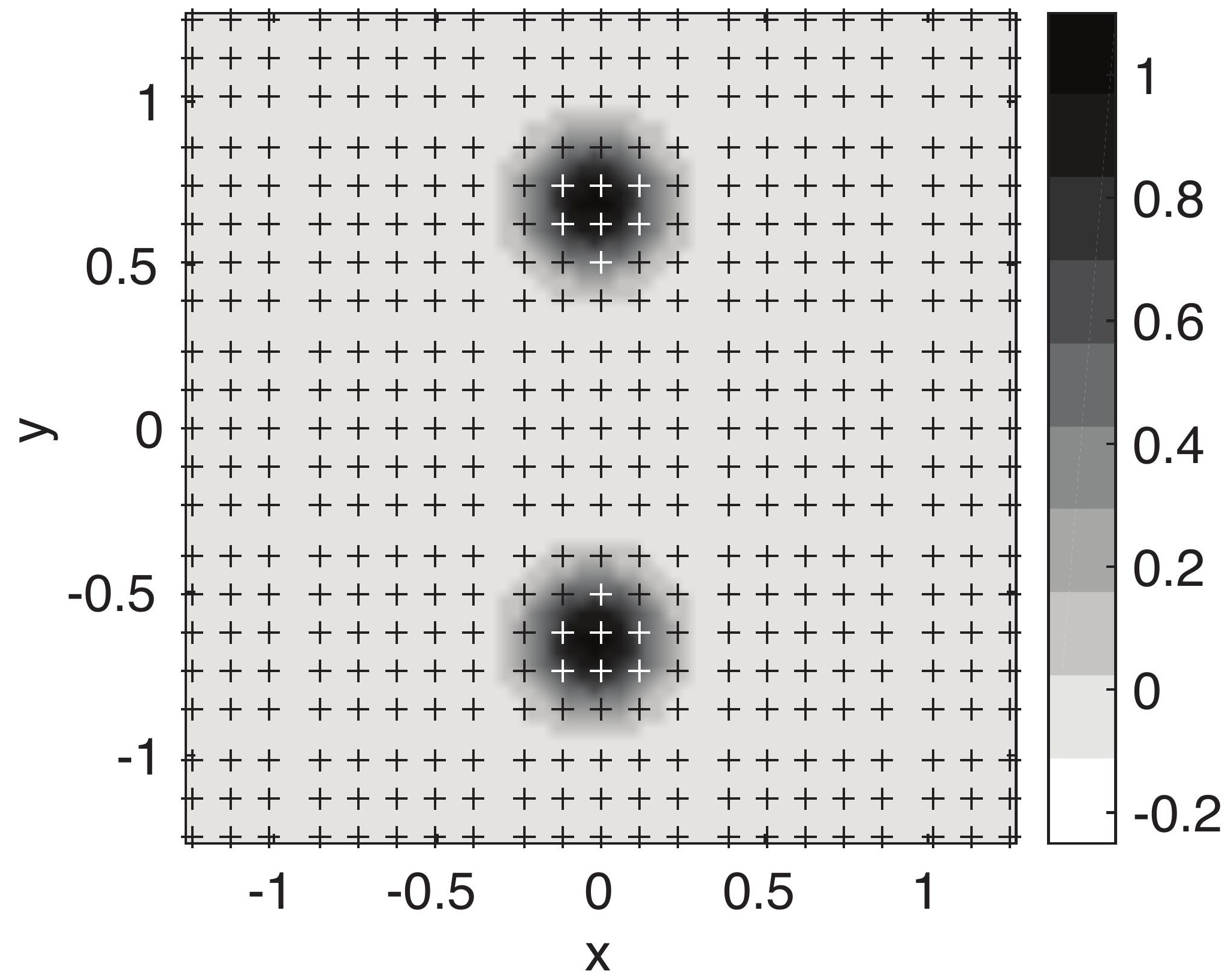




\section{$\mathrm{t}=60 / 300$}

t+t+t+t+t+t+t+t+t+t+t $+++++++++++++++++++$

$1 \mathrm{t}++++++++++++++++++t$

$+++++++++++++++++++$ $t++++++++++++++++++t$

$0.5 t++++++++++++++++++t$

1
0.8

$0.5++++++++++++++++++++t$ $++++++++++++++++++++$ $++++++++++++++++++++$ $t+++++++++++++++++++$

$>00 t+++++++++++++++++++t$ $+++++++++++++++++++t$ $t+++++++++++++++++++$

$0.5++++++++++++++++++++$

$-0.5+++++++++++++++++++++$ $++++++++++++++++++++$ $++++++++++++++++++++$ $t+++++++++++++++++++$

-1 $1++++++++++++++++++++$ $++++++++++++++++++++$ $4+t+t+t+t+t+t+t+t+4+t$

0.6

$$
\begin{array}{lllll}
-1 & -0.5 & 0 & 0.5 & 1
\end{array}
$$




\section{$\mathrm{t}=120 / 300$}

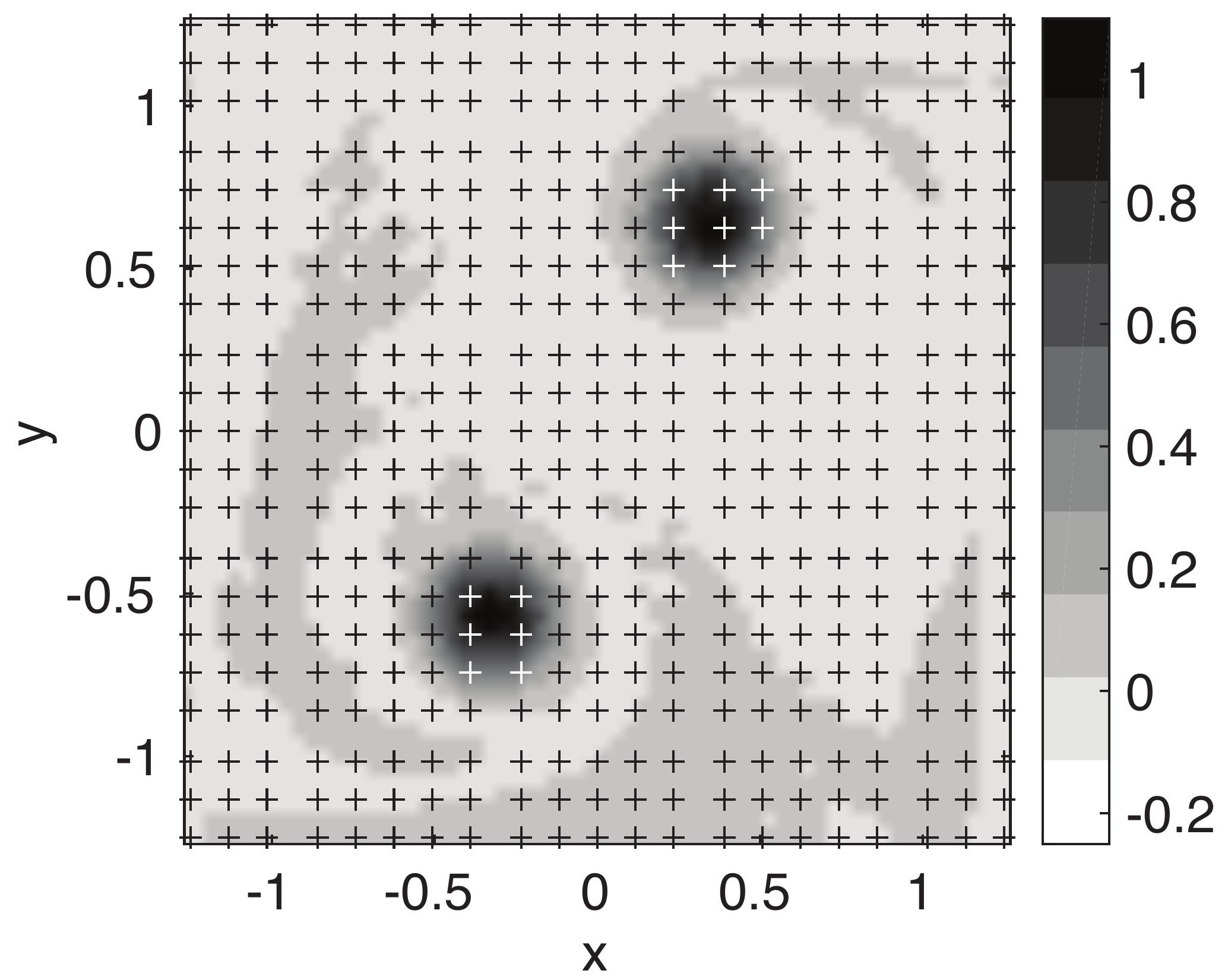




\section{$t=180 / 300$}

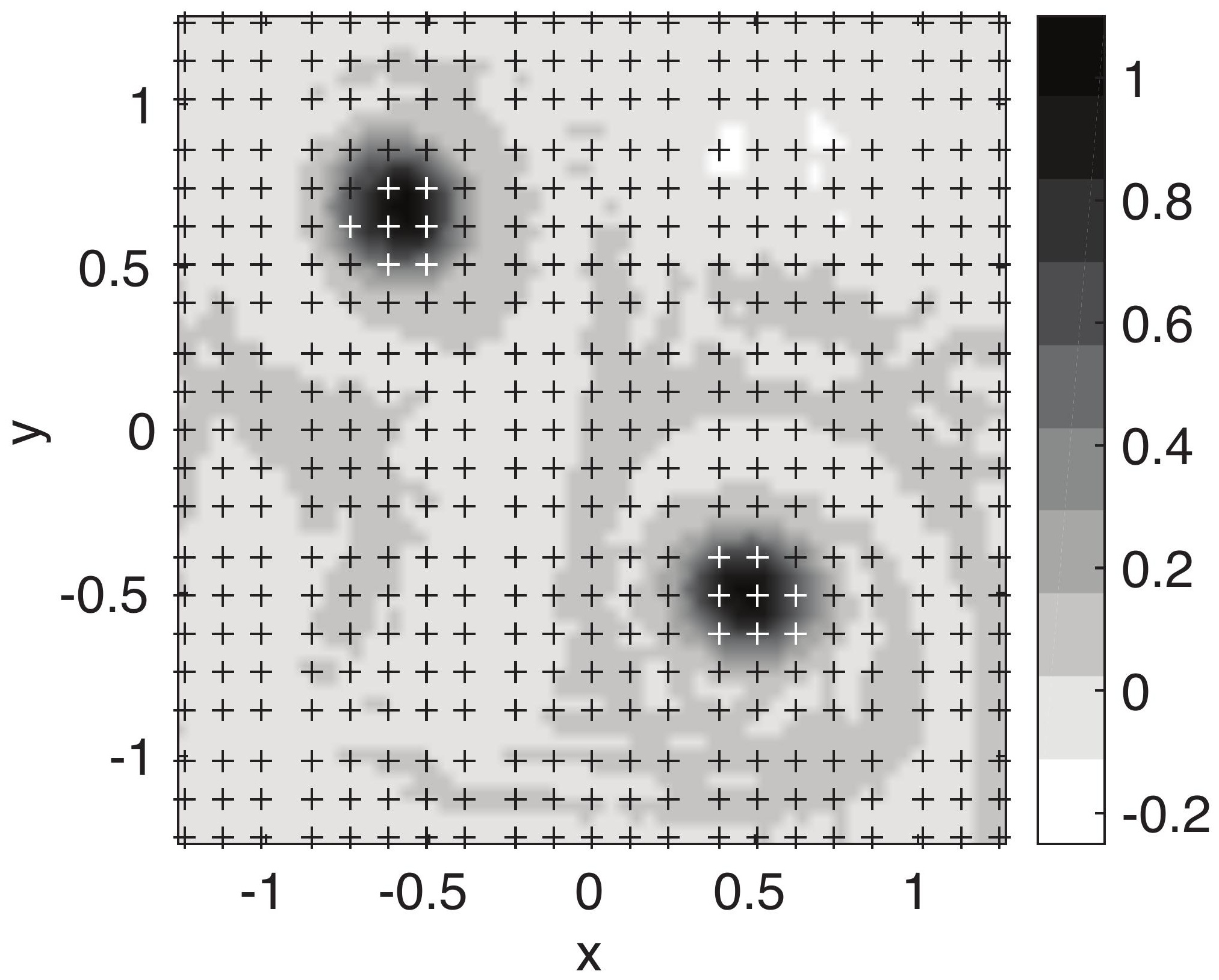




\section{$\mathrm{t}=240 / 300$}

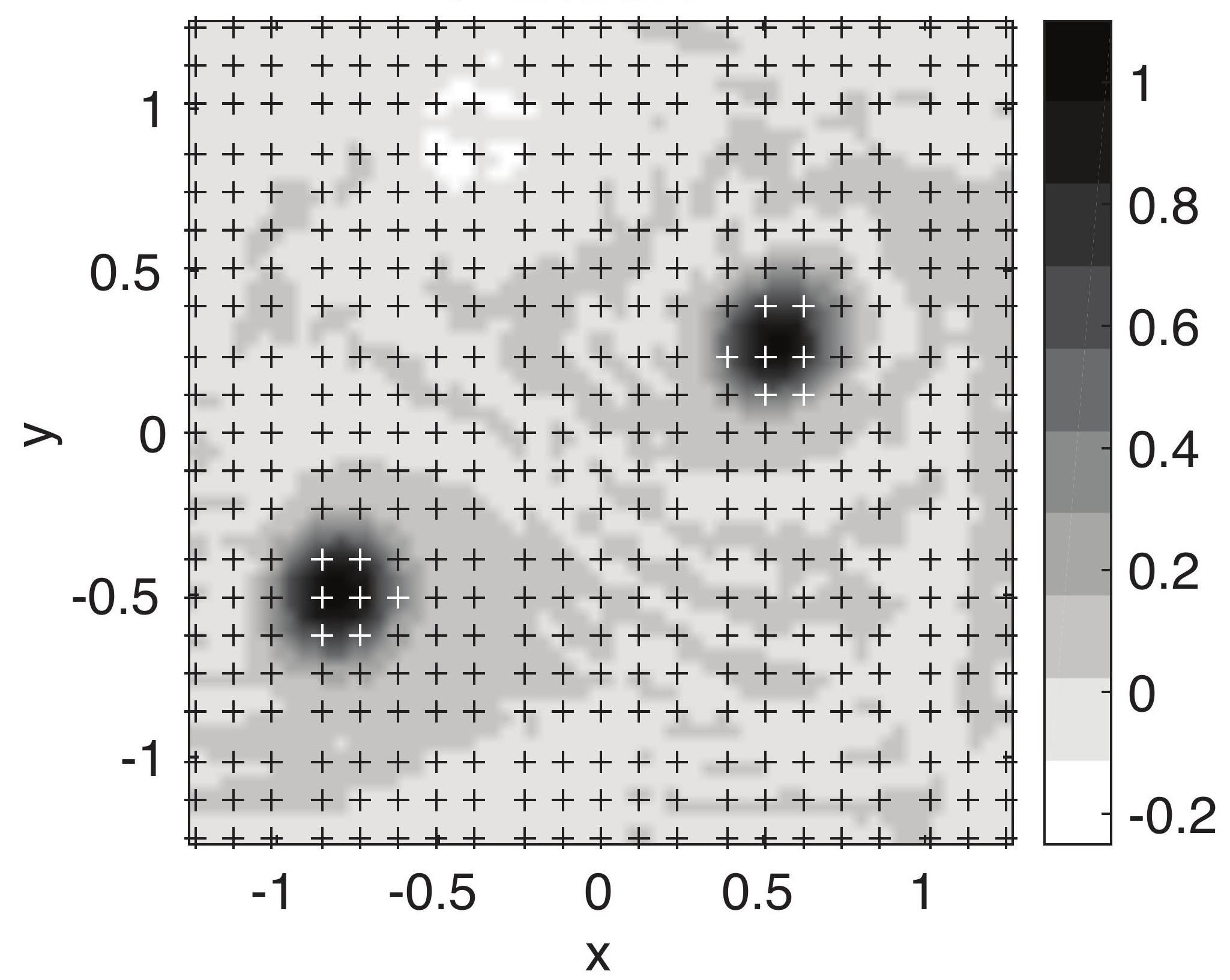




\section{$\mathrm{t}=300 / 300$}

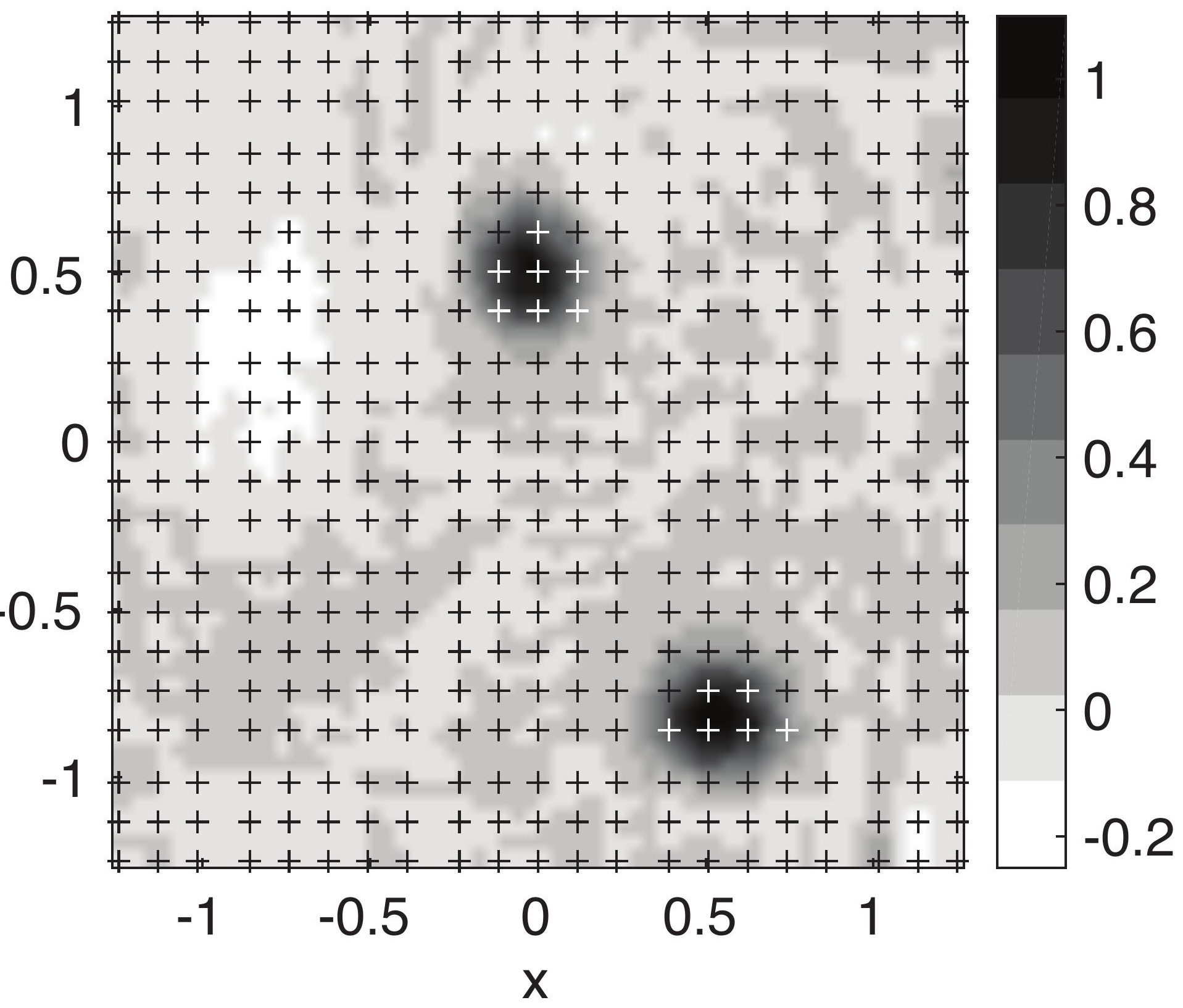


Figure 51 of 6

$t=0 / 300$
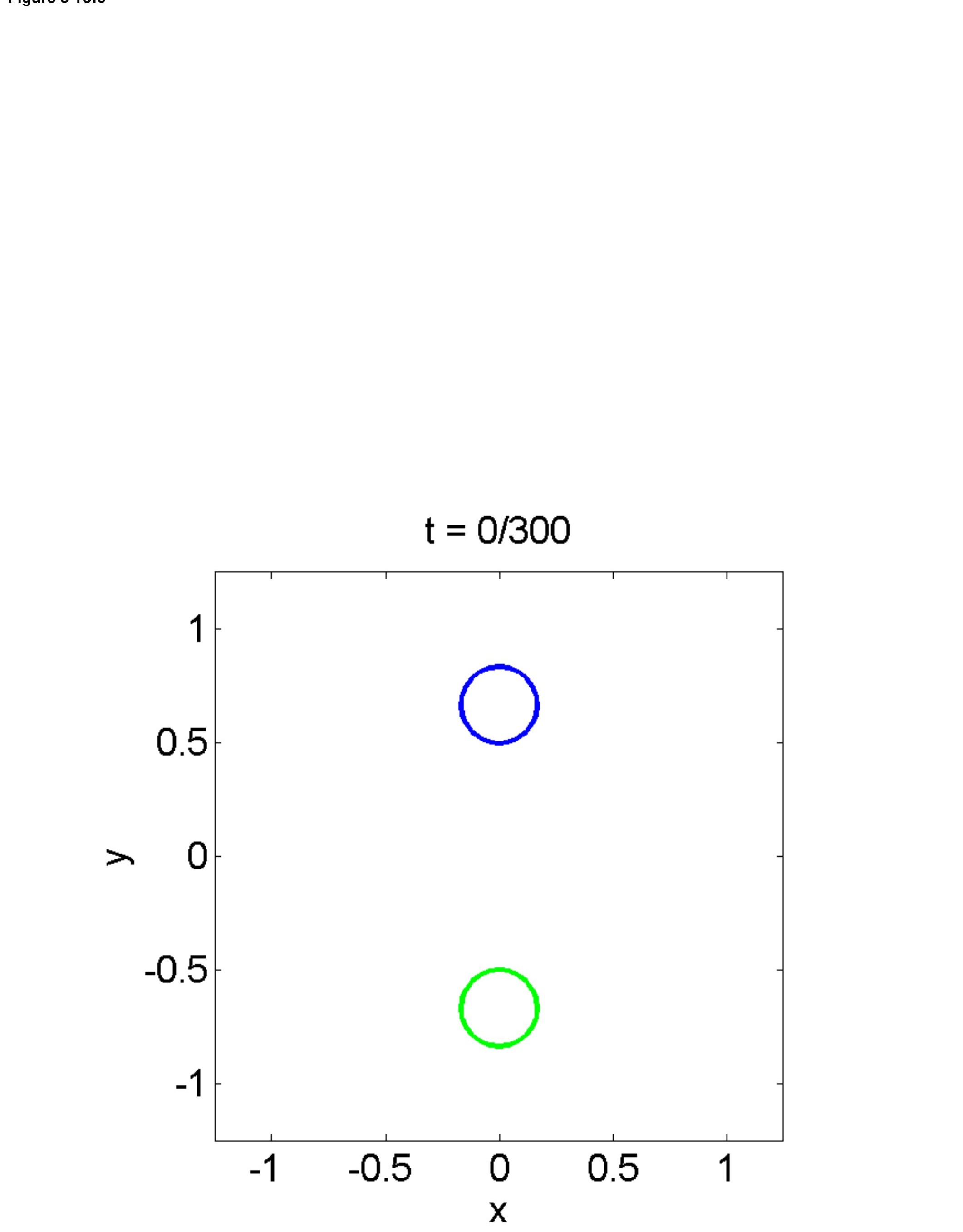
Figure 52 of 6

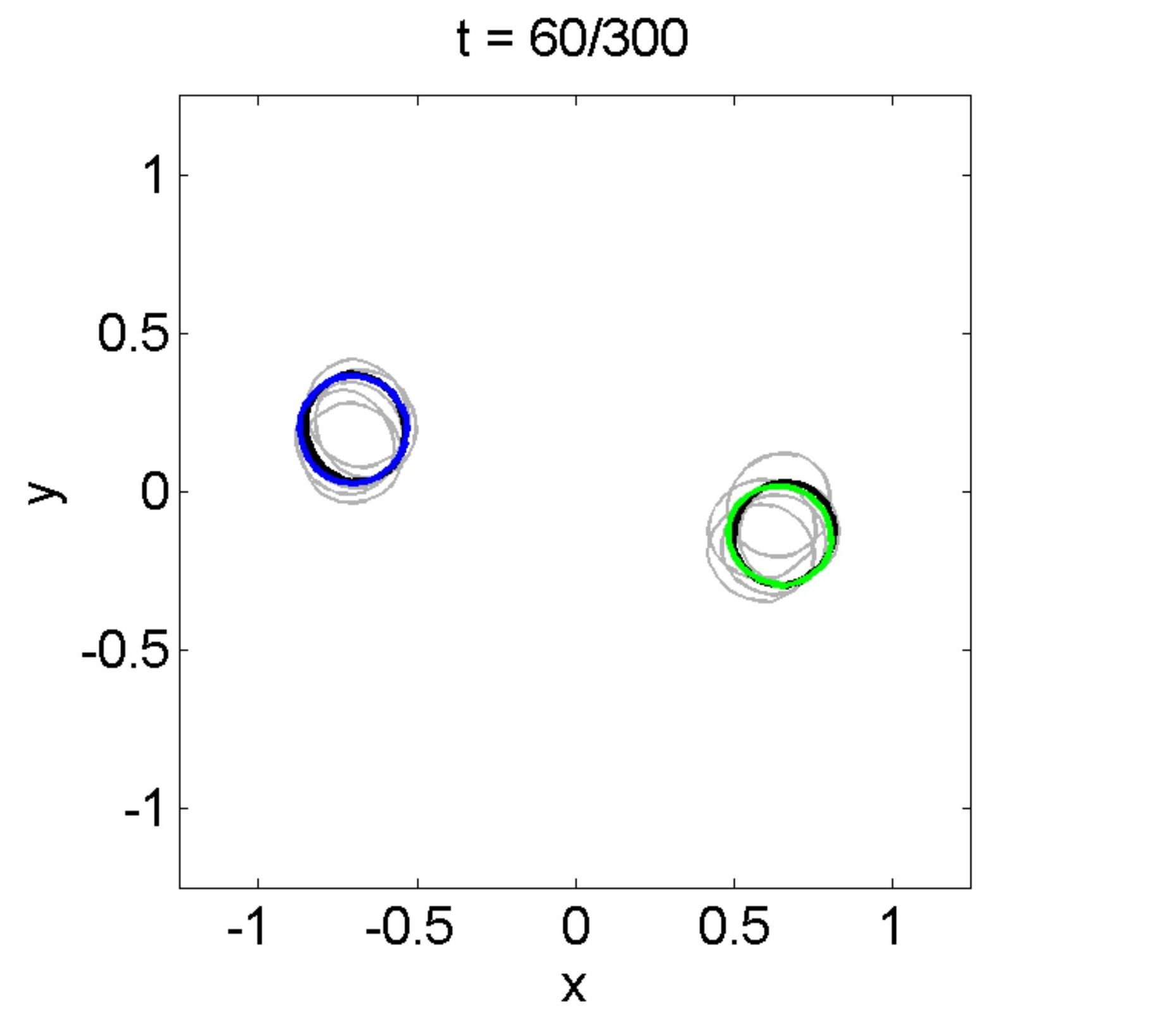

\section{$t=60 / 300$}
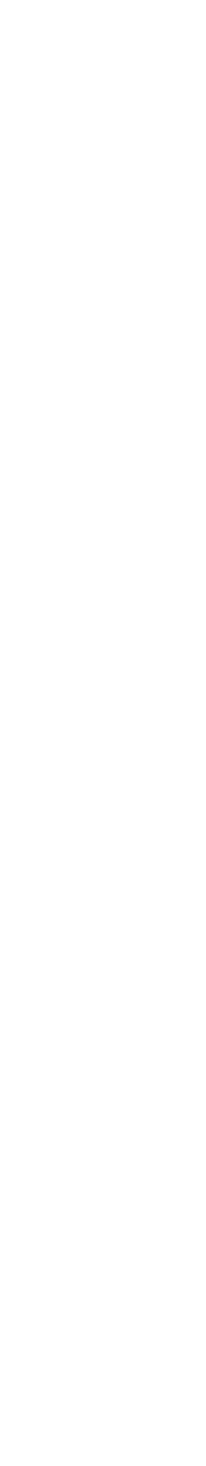

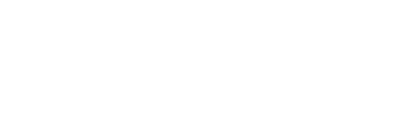


Figure 53 of 6
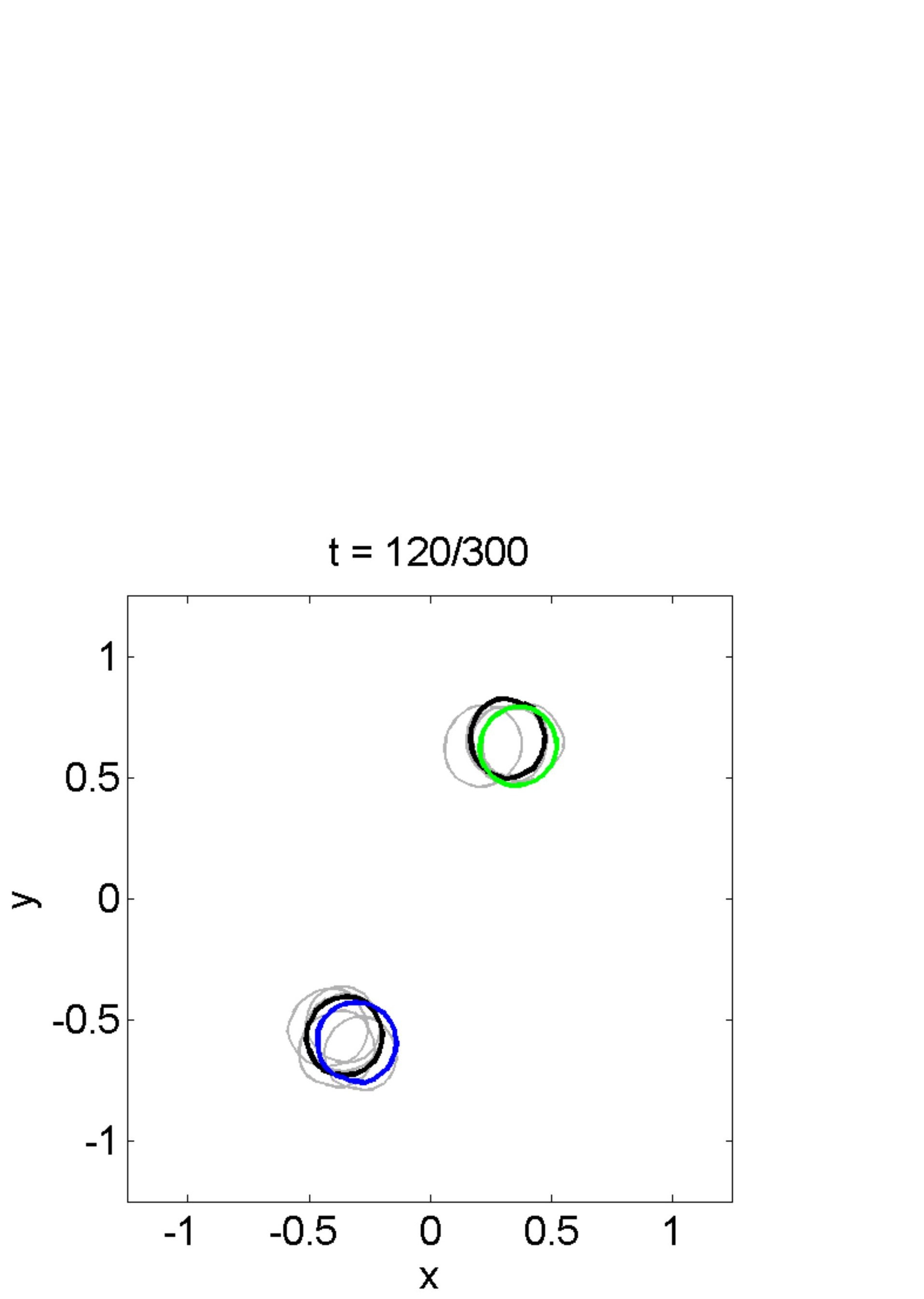
. . 
Figure 5 4of6

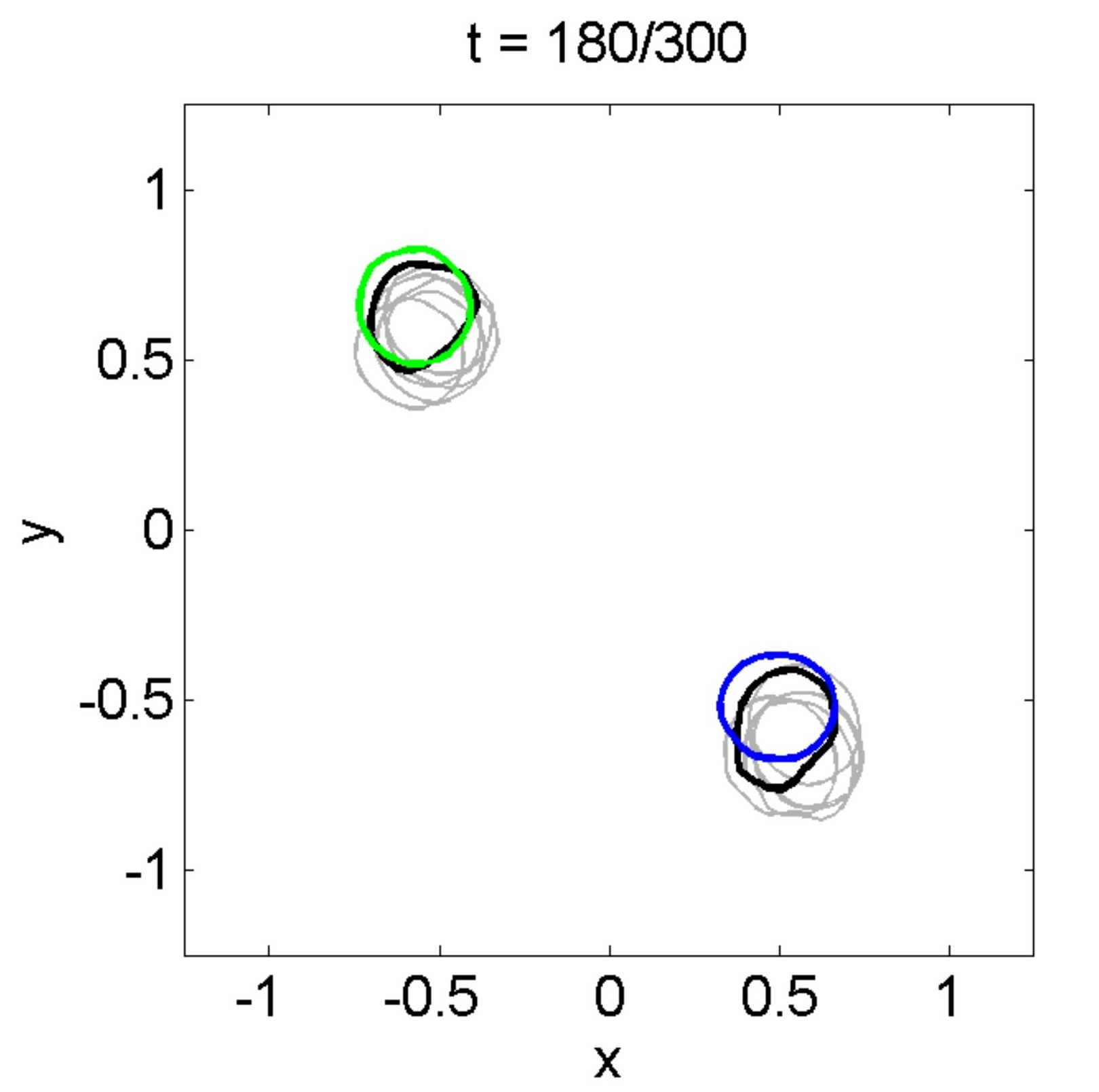

$t=180 / 300$

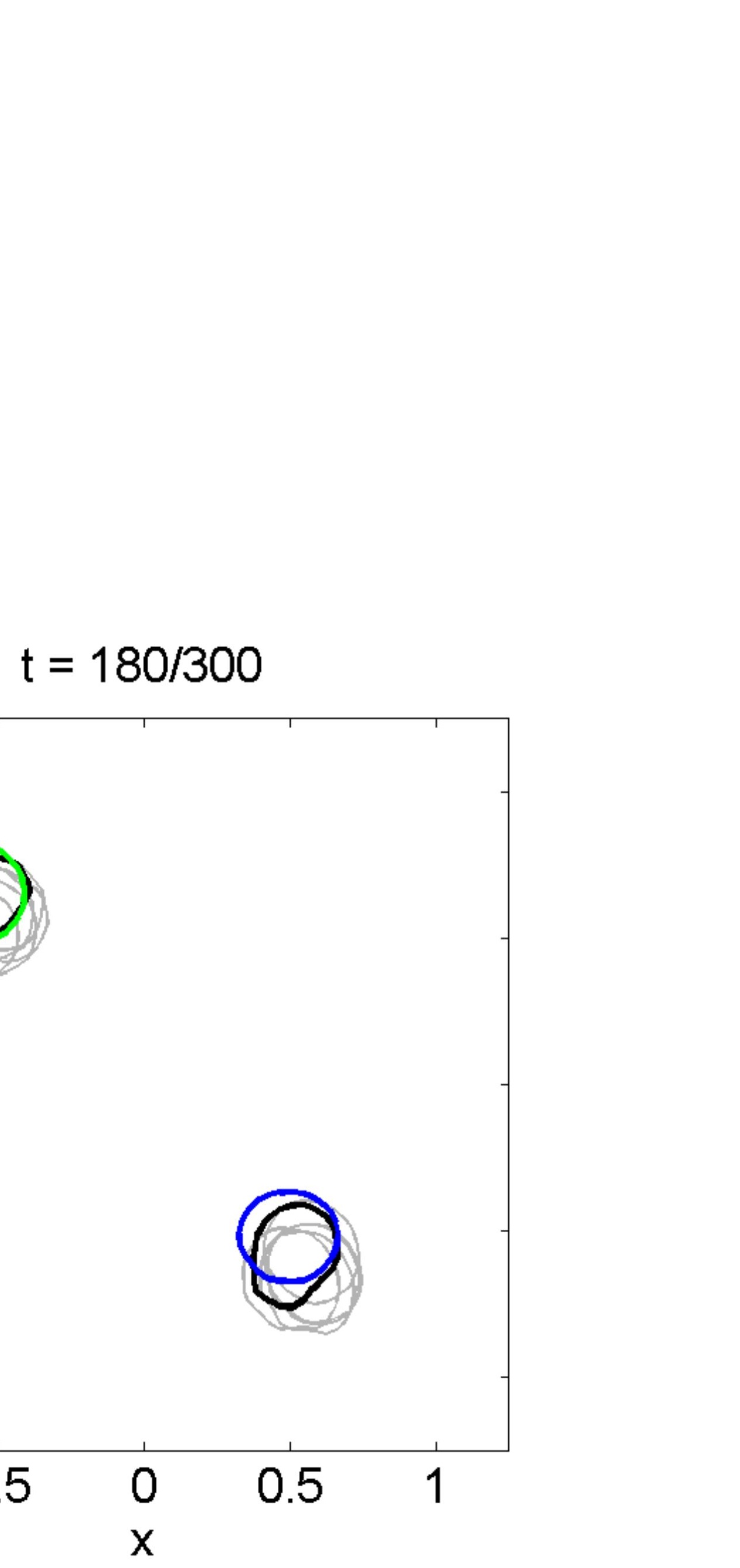


Figure 5 5of6
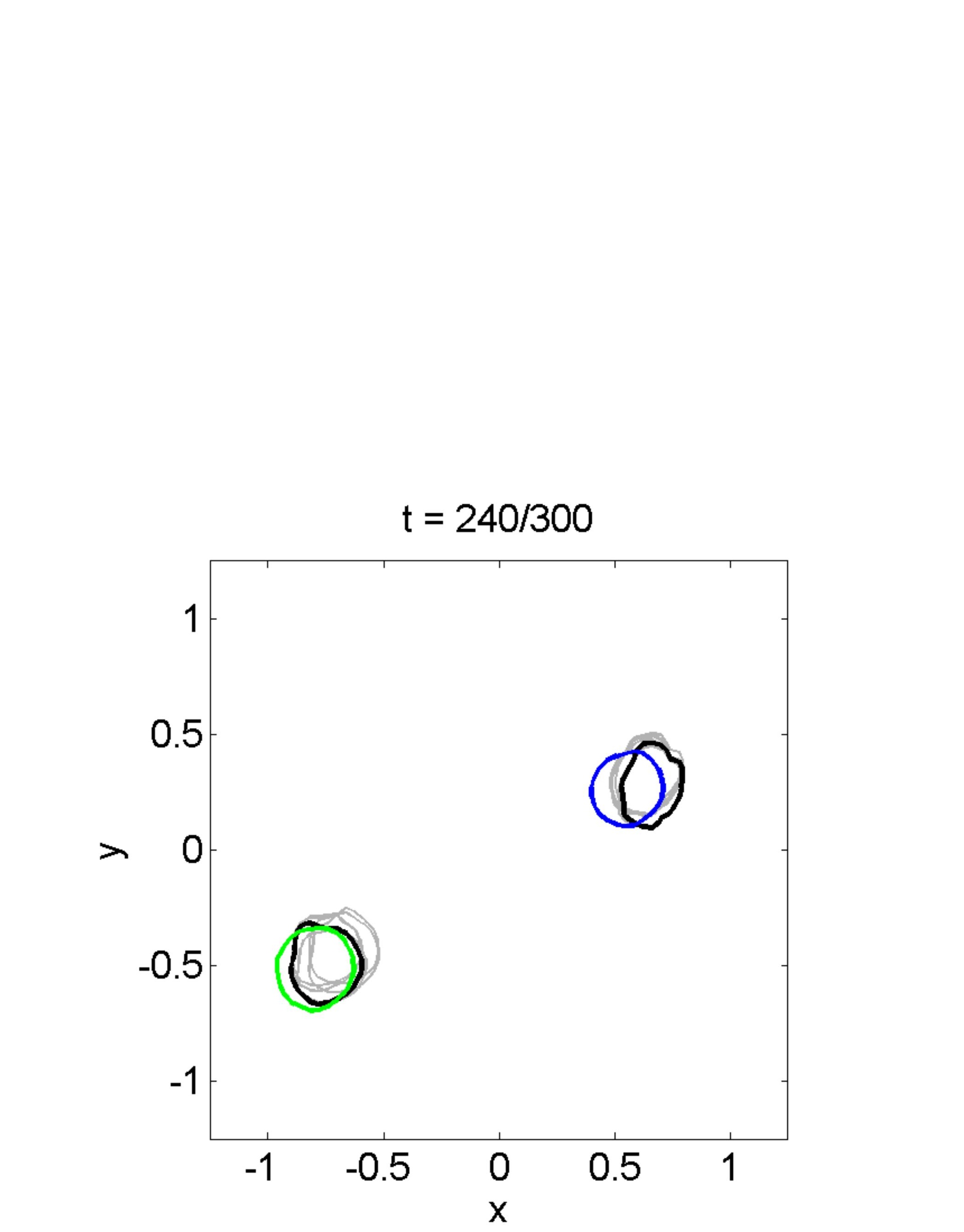
Hod

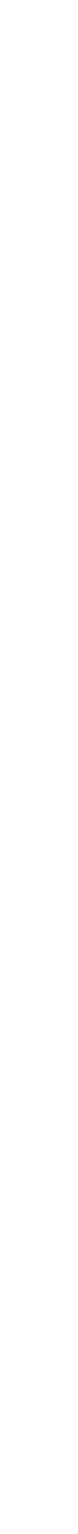


Figure 56 of 6
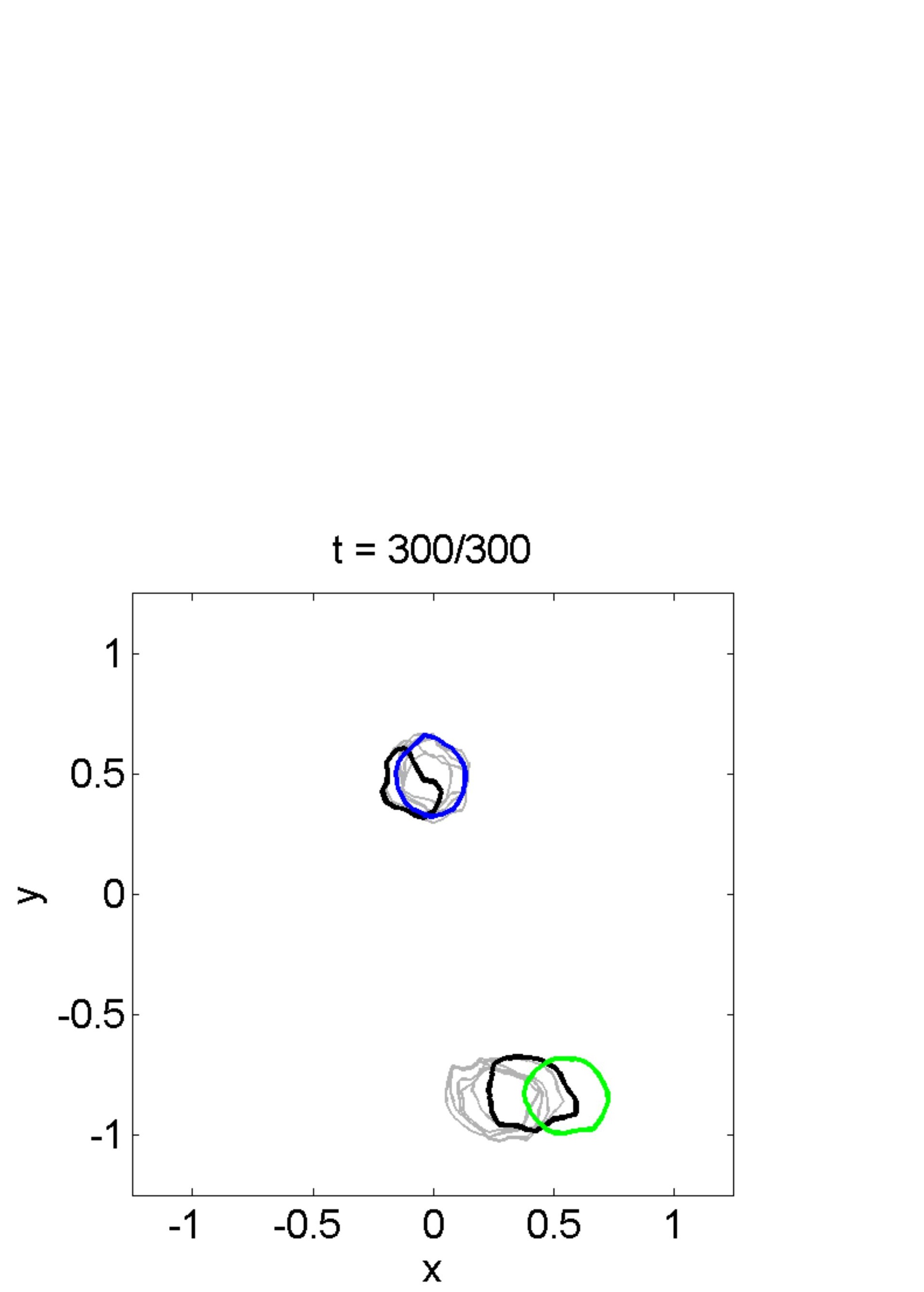

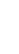 . \\ .}


Figure 61 of 6

$t=0 / 300$
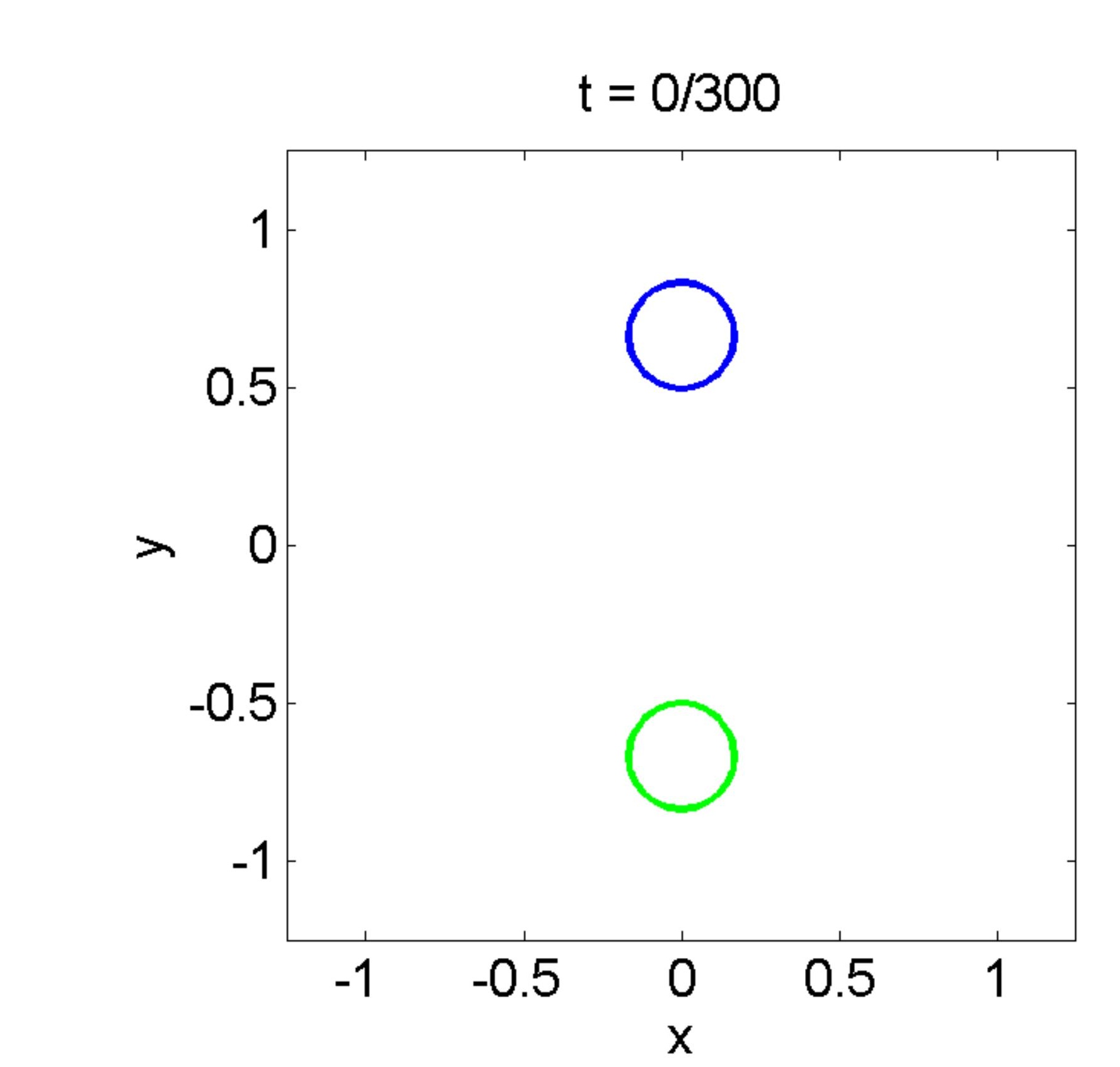
(2)
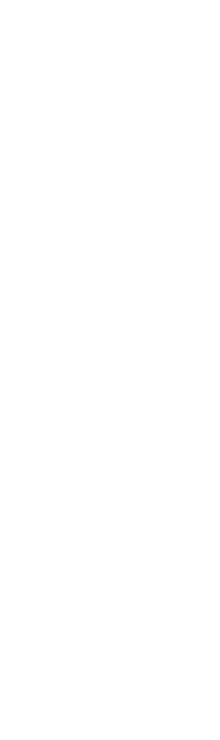

6

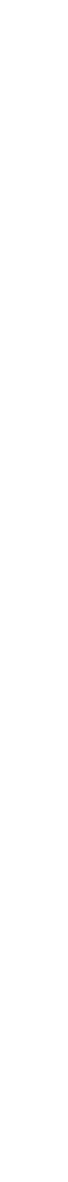


Figure 6 2of6

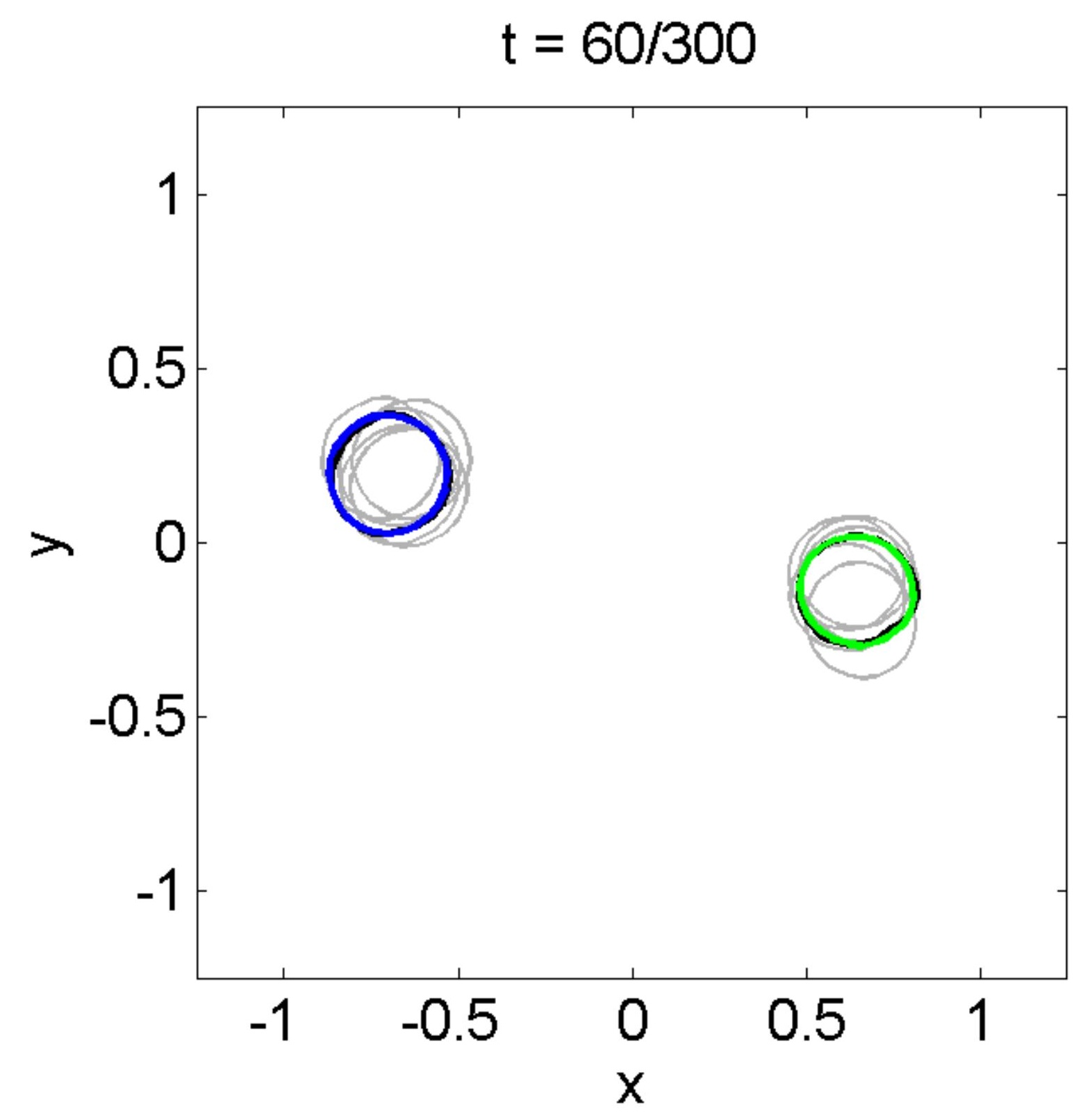

$t=60 / 300$
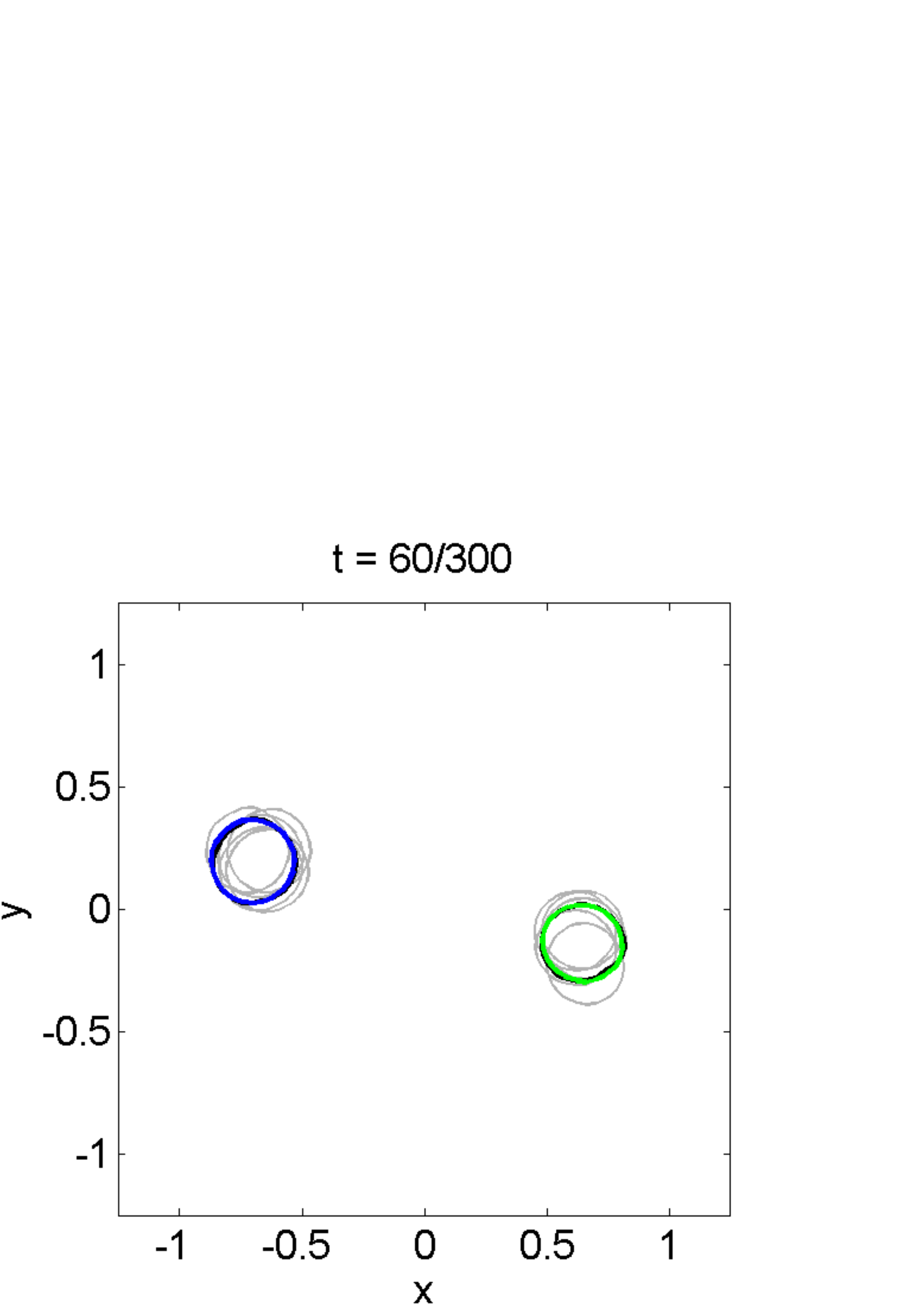
Figure $630 f 6$

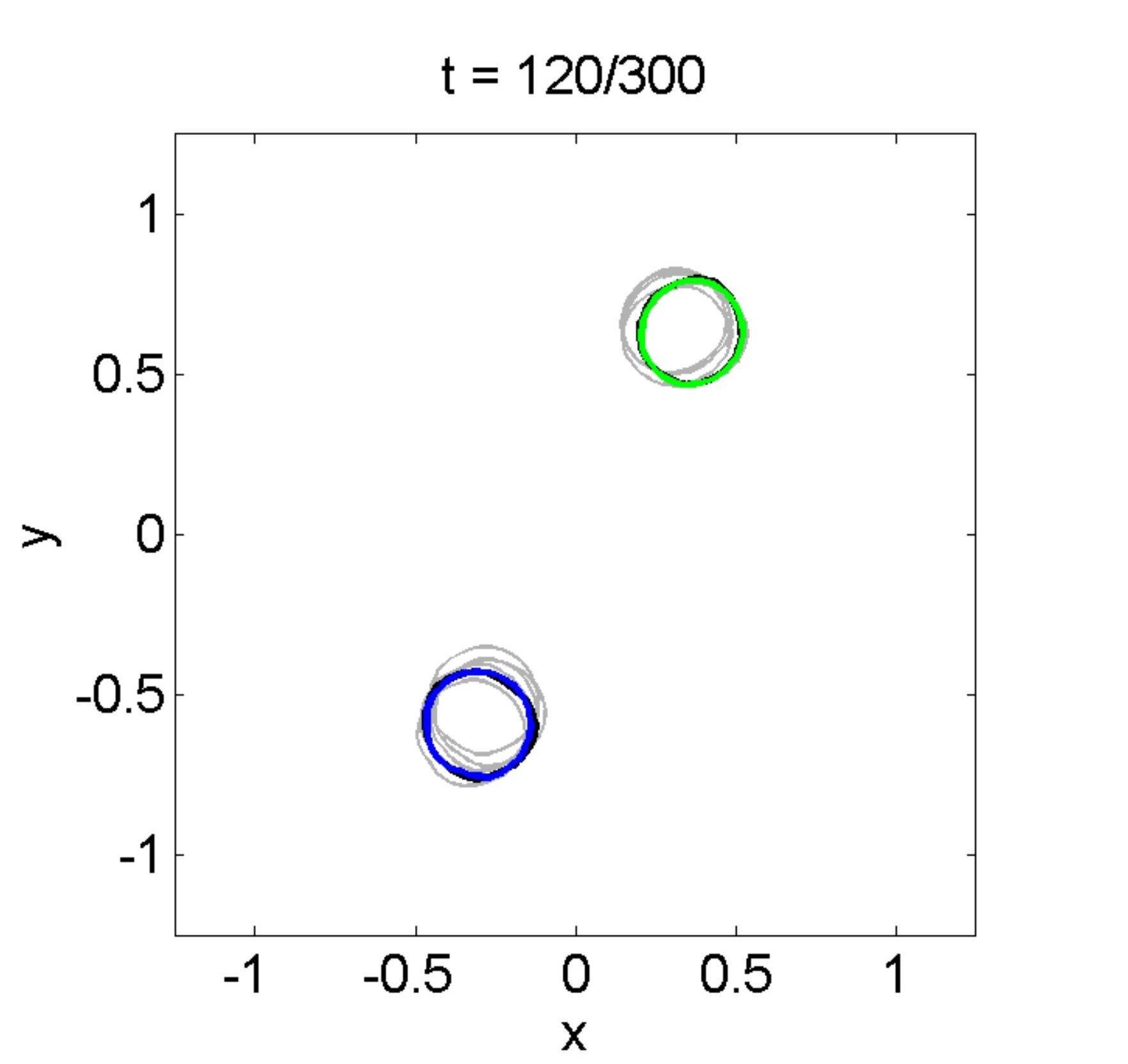

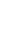
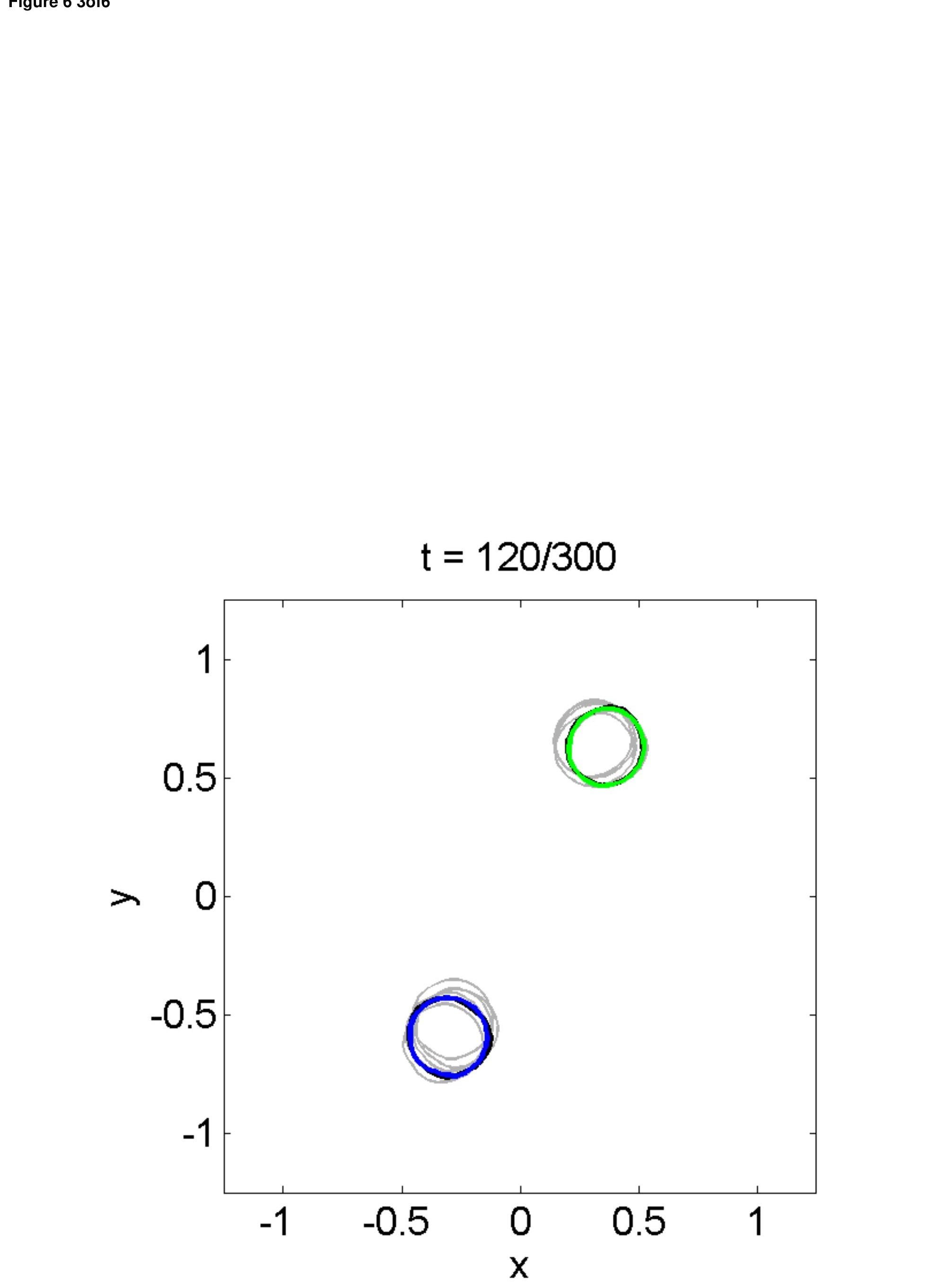
Figure 640 6
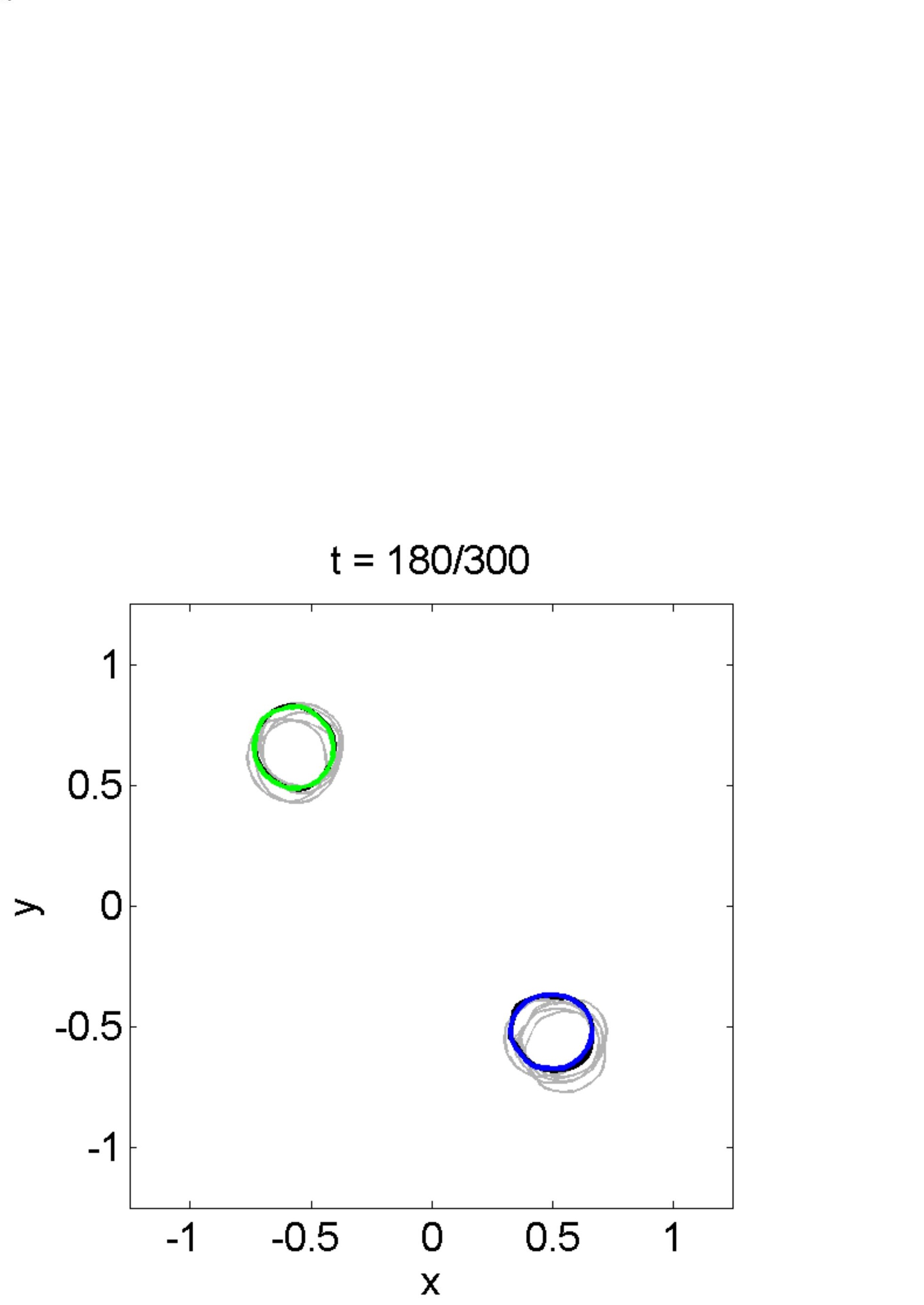
Figure $650 f 6$
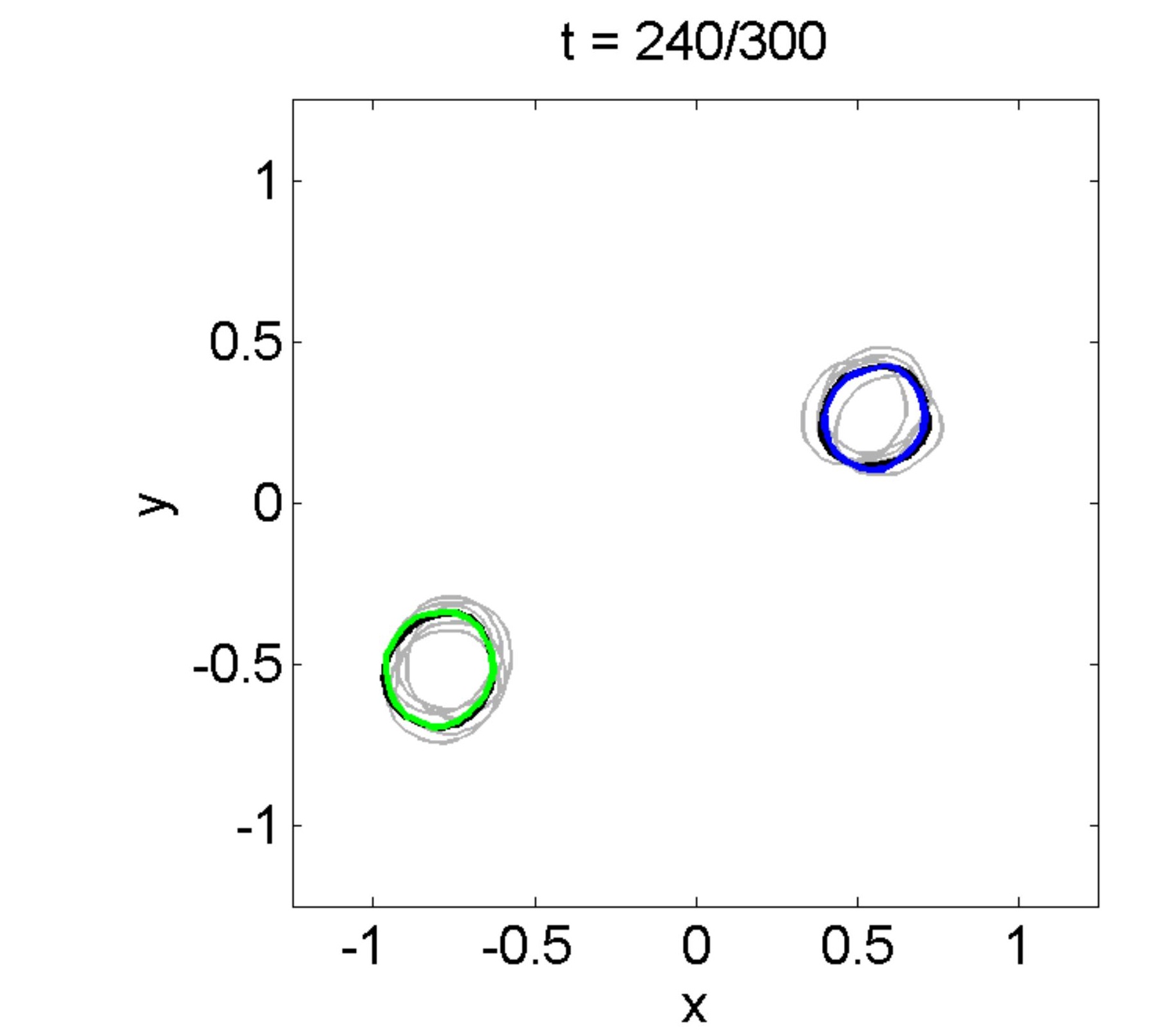
Figure 66 of 6
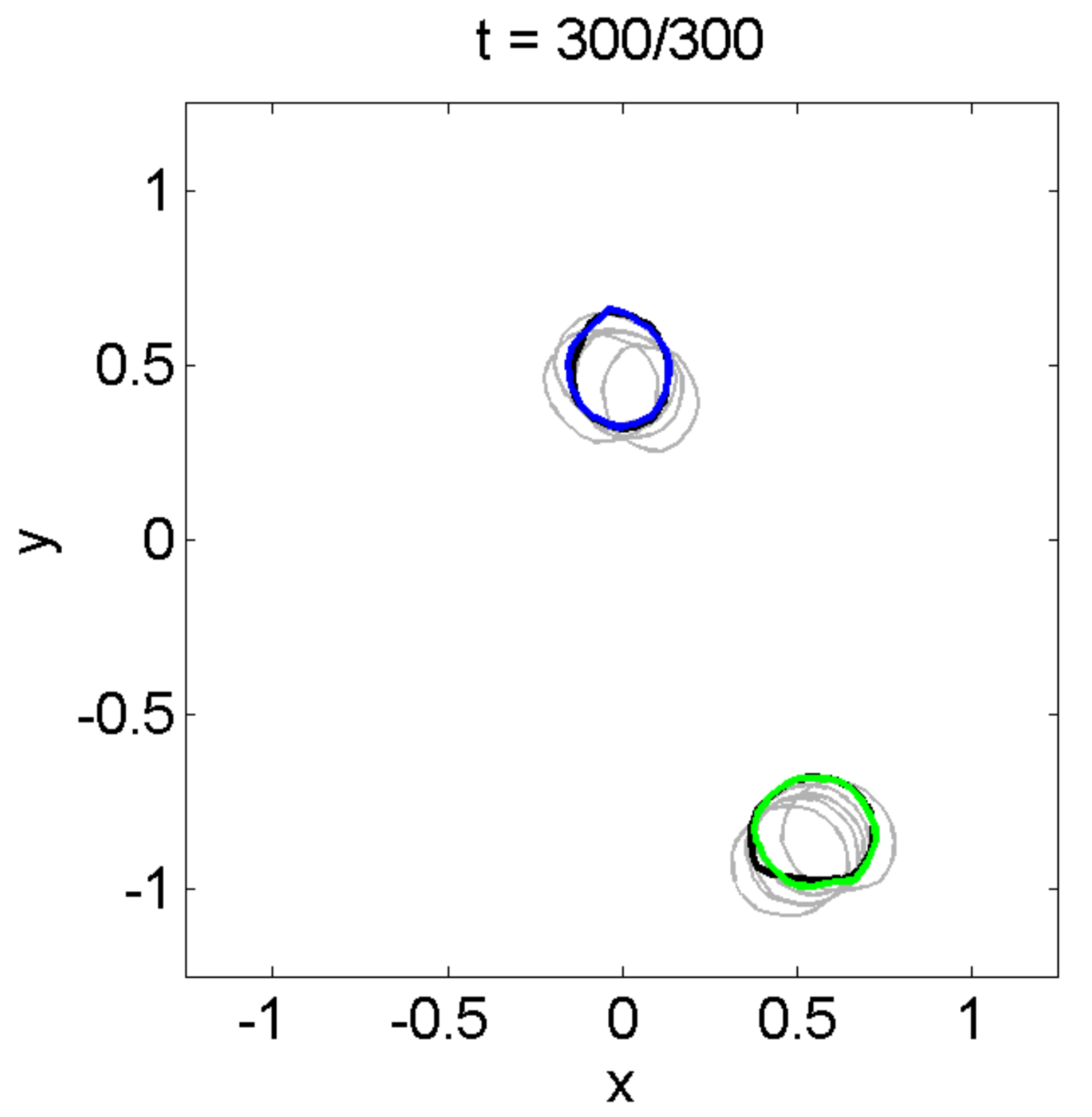

$$
t=300 / 300
$$

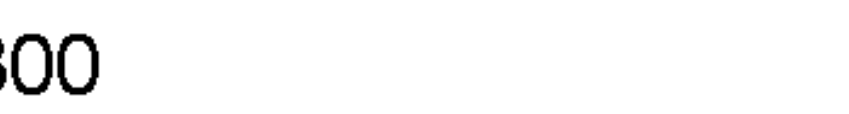


Figure 71 of 2
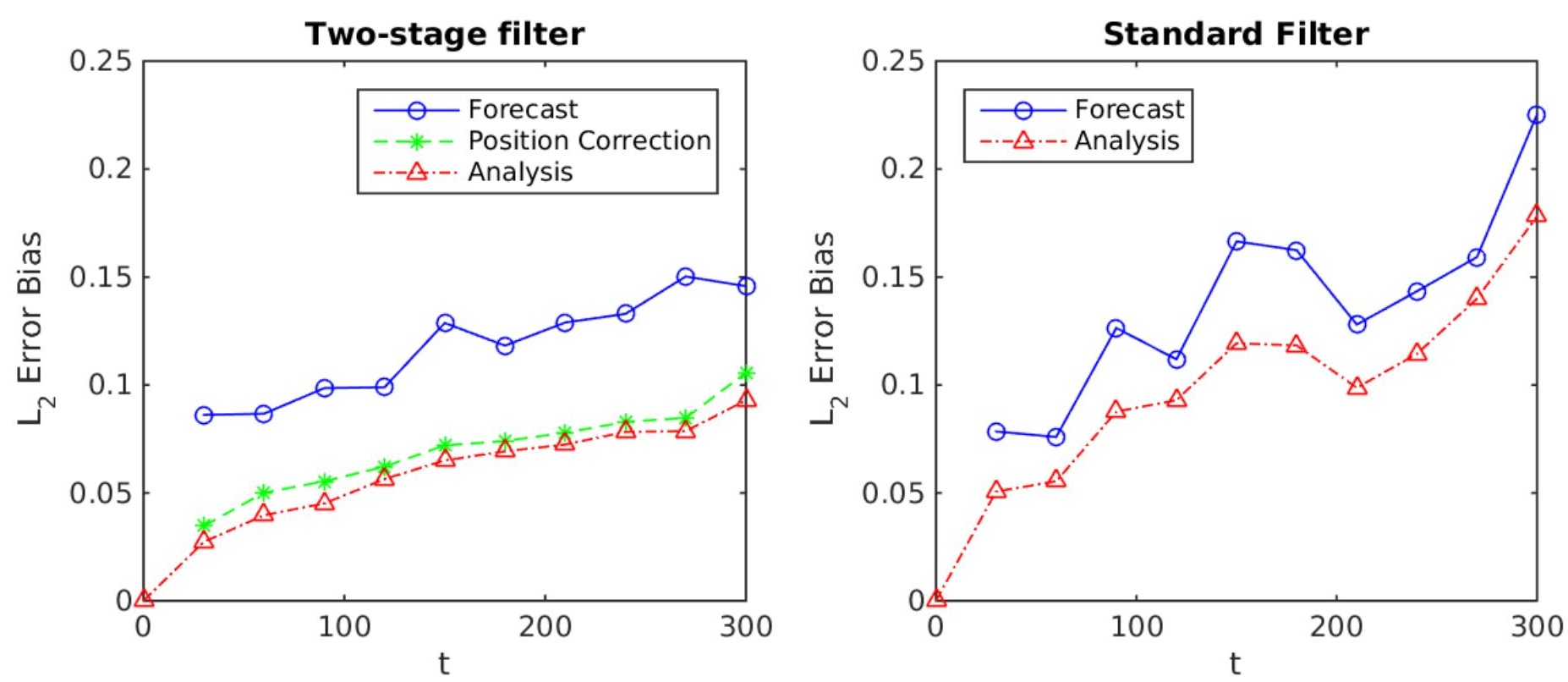

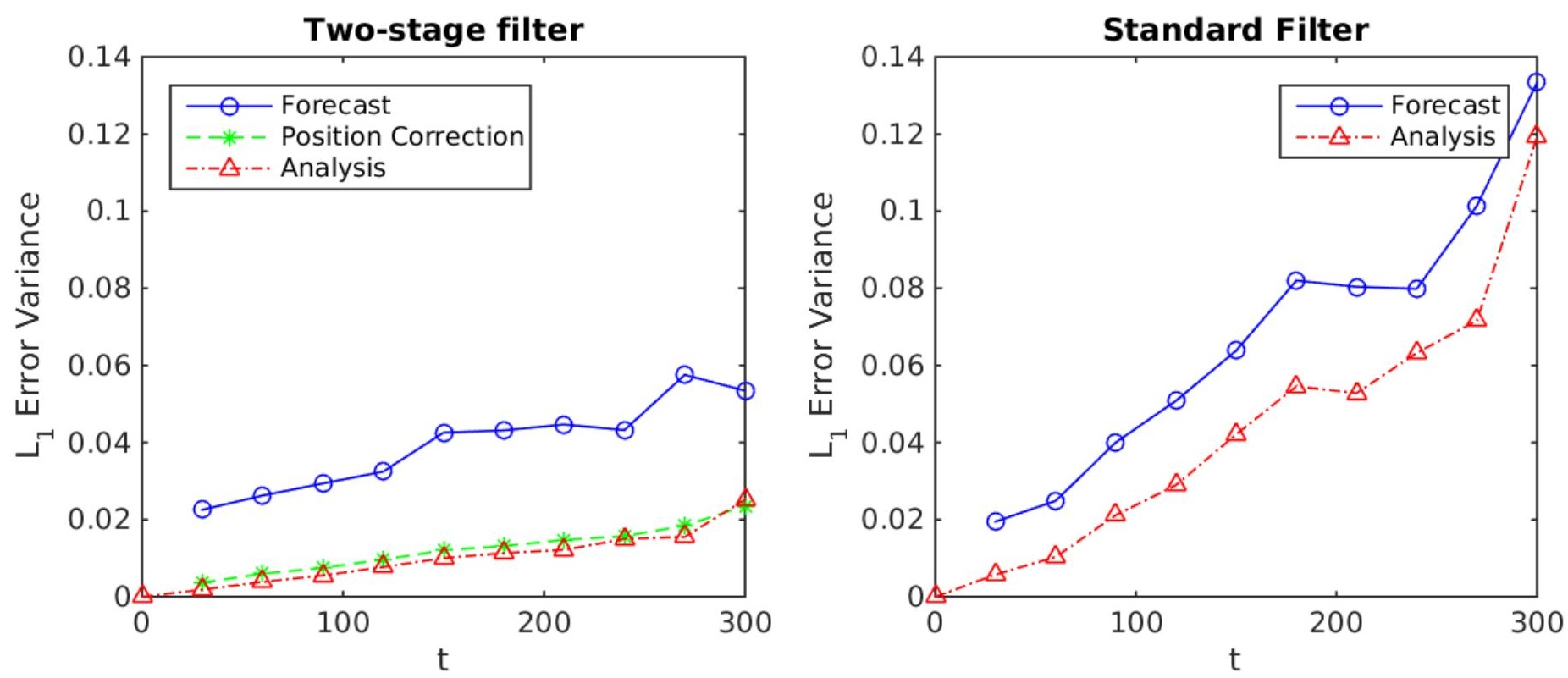
Figure 81 of 2
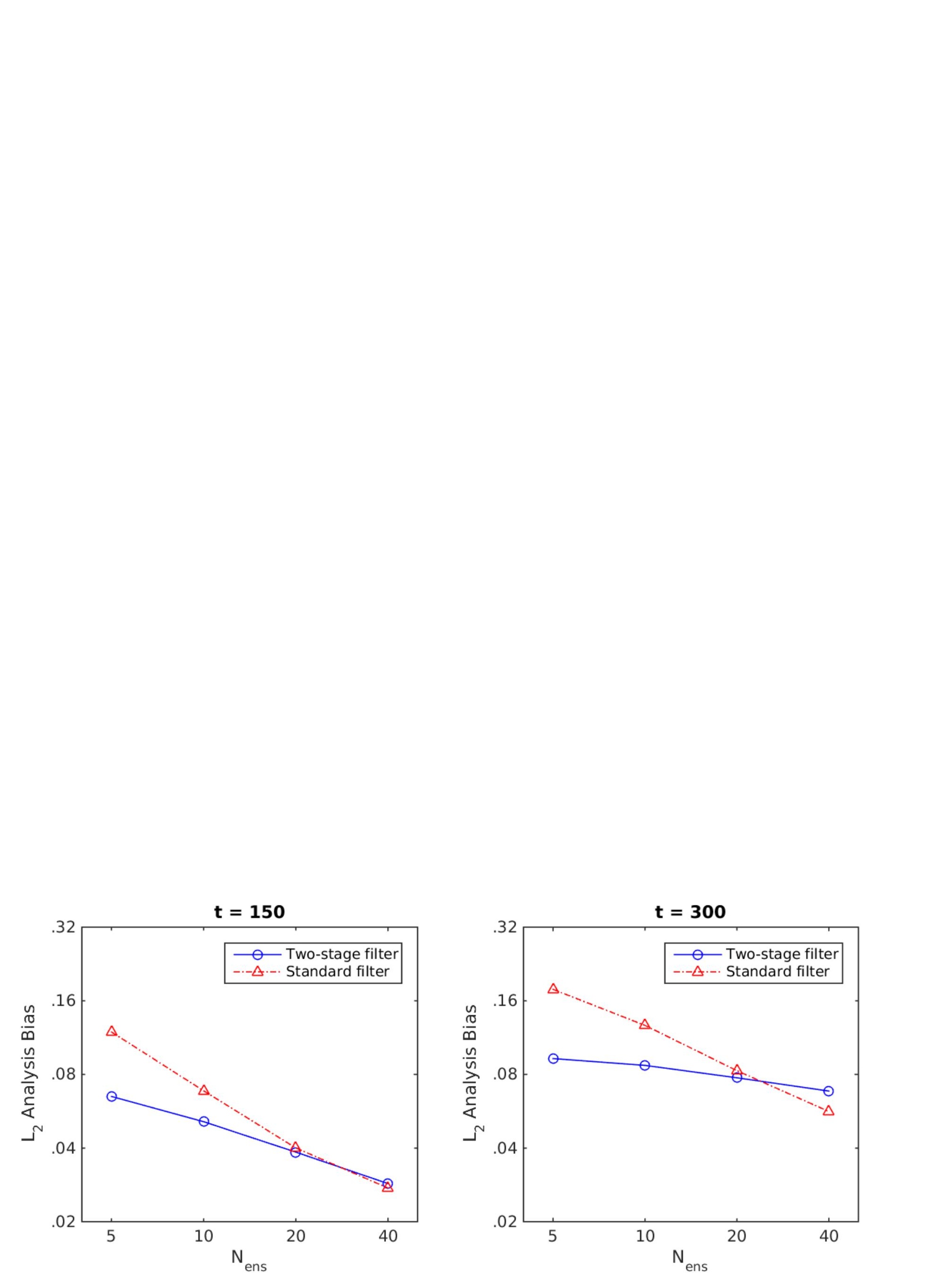

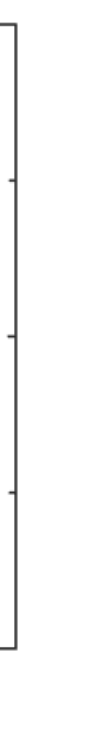


Figure 91 of 2
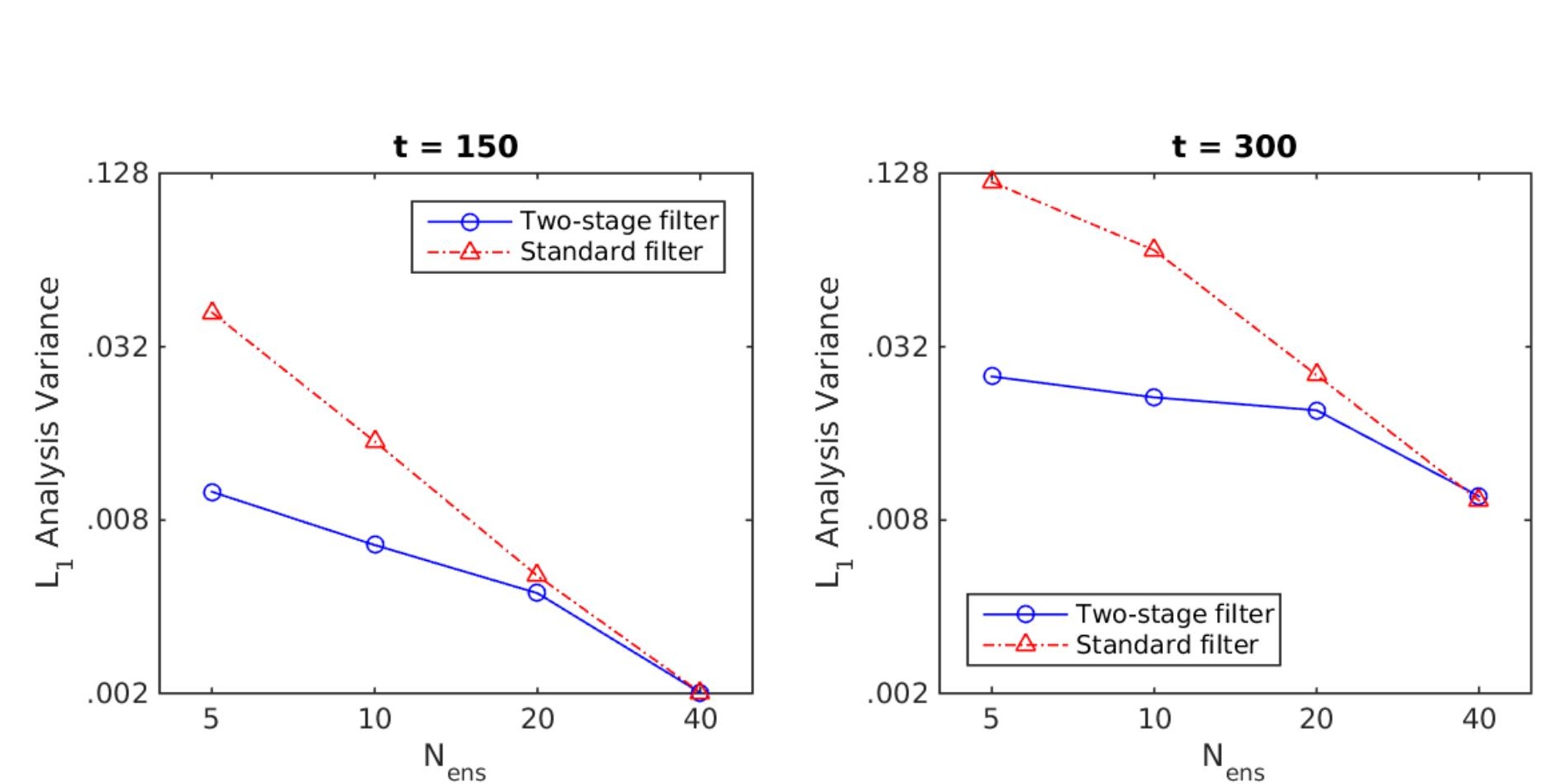

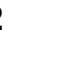

\section{Fure 910}

ens 
